\title{
Search for direct production of electroweakinos in final states with one lepton, missing transverse momentum and a Higgs boson decaying into two $b$-jets in $p p$ collisions at $\sqrt{s}=13 \mathrm{TeV}$ with the ATLAS detector
}

\author{
ATLAS Collaboration ${ }^{\star}$ \\ CERN, 1211 Geneva 23, Switzerland
}

Received: 20 September 2019 / Accepted: 15 May 2020 / Published online: 1 August 2020

(C) CERN for the benefit of the ATLAS collaboration 2020

\begin{abstract}
The results of a search for electroweakino pair production $p p \rightarrow \tilde{\chi}_{1}^{ \pm} \tilde{\chi}_{2}^{0}$ in which the chargino $\left(\tilde{\chi}_{1}^{ \pm}\right)$decays into a $W$ boson and the lightest neutralino $\left(\tilde{\chi}_{1}^{0}\right)$, while the heavier neutralino $\left(\tilde{\chi}_{2}^{0}\right)$ decays into the Standard Model 125 $\mathrm{GeV}$ Higgs boson and a second $\tilde{\chi}_{1}^{0}$ are presented. The signal selection requires a pair of $b$-tagged jets consistent with those from a Higgs boson decay, and either an electron or a muon from the $W$ boson decay, together with missing transverse momentum from the corresponding neutrino and the stable neutralinos. The analysis is based on data corresponding to $139 \mathrm{fb}^{-1}$ of $\sqrt{s}=13 \mathrm{TeV} p p$ collisions provided by the Large Hadron Collider and recorded by the ATLAS detector. No statistically significant evidence of an excess of events above the Standard Model expectation is found. Limits are set on the direct production of the electroweakinos in simplified models, assuming pure wino cross-sections. Masses of $\tilde{\chi}_{1}^{ \pm} / \tilde{\chi}_{2}^{0}$ up to $740 \mathrm{GeV}$ are excluded at $95 \%$ confidence level for a massless $\tilde{\chi}_{1}^{0}$.
\end{abstract}

\section{Introduction}

The Standard Model (SM) is a remarkably successful theory, yet it is clear that this theory is not a complete description of nature. The discovery in 2012 of the SM Higgs boson [1-4], by the ATLAS and CMS collaborations, confirmed the mechanism of the electroweak symmetry breaking and highlighted the hierarchy problem [5-8]. Supersymmetry (SUSY) [914], a theoretical extension to the SM, resolves the hierarchy problem by introducing a new fermion (boson) supersymmetric partner for each boson (fermion) in the SM. In SUSY models that conserve $R$-parity [15], the SUSY particles are produced in pairs. Furthermore, the lightest supersymmetric particle (LSP) is stable and weakly interacting, thus constituting a viable dark-matter candidate $[16,17]$.

^e-mail: atlas.publications@ @ern.ch
In SUSY scenarios the partners of the SM Higgs boson (h) and the gauge bosons, known as the higgsinos, winos (partners of the SU(2)L gauge fields), and bino (partner of the $\mathrm{U}(1)$ gauge field) are collectively referred to as electroweakinos. Charginos $\tilde{\chi}_{i}^{ \pm}(i=1,2)$ and neutralinos $\tilde{\chi}_{j}^{0}$ $(j=1,2,3,4)$ are the electroweakino mass eigenstates which are linear superpositions of higgsinos, winos, and bino. For the models considered in this paper, the lightest neutralino $\left(\tilde{\chi}_{1}^{0}\right)$ is a bino-like LSP. The lightest chargino $\left(\tilde{\chi}_{1}^{ \pm}\right)$ and next-to-lightest neutralino $\left(\tilde{\chi}_{2}^{0}\right)$ are wino-like and nearly mass degenerate.

Naturalness considerations $[18,19]$ suggest that the lightest of the electroweakinos have masses near the electroweak scale. In scenarios where the strongly produced SUSY particles are heavier than a few $\mathrm{TeV}$, the direct production of electroweakinos may be the dominant SUSY production mechanism at the Large Hadron Collider (LHC). The lightest chargino and next-to-lightest neutralino can decay via $\tilde{\chi}_{1}^{ \pm} \rightarrow W \tilde{\chi}_{1}^{0}$ and $\tilde{\chi}_{2}^{0} \rightarrow h / Z \tilde{\chi}_{1}^{0}$ respectively [20-22] in scenarios where the lepton superpartners are heavier than the electroweakinos. In this case the decay via the Higgs boson is dominant for many choices of SUSY parameters, as long as $m\left(\tilde{\chi}_{2}^{0}\right)-m\left(\tilde{\chi}_{1}^{0}\right)>m(h)$. Scenarios with light electroweakinos also provide a possible explanation for the discrepancy between the muon anomalous magnetic moment $g-2$ measurement and the SM predictions [23,24].

This paper presents a search for direct production of electroweakinos in proton-proton $(p p)$ collisions produced at the LHC at $\sqrt{s}=13 \mathrm{TeV}$. This analysis is designed to be sensitive to direct production of a chargino and a neutralino that promptly decay as $\tilde{\chi}_{1}^{ \pm} \rightarrow W \tilde{\chi}_{1}^{0}$ and $\tilde{\chi}_{2}^{0} \rightarrow h \tilde{\chi}_{1}^{0}$. The search targets a $W$ boson which decays into an electron or muon (and corresponding neutrino) and a Higgs boson which decays into a pair of $b$-quarks, as shown in Fig. 1. The signature consists of exactly one light lepton $(e$ or $\mu$ ), two jets originating from the fragmentation of $b$-quarks, and missing 


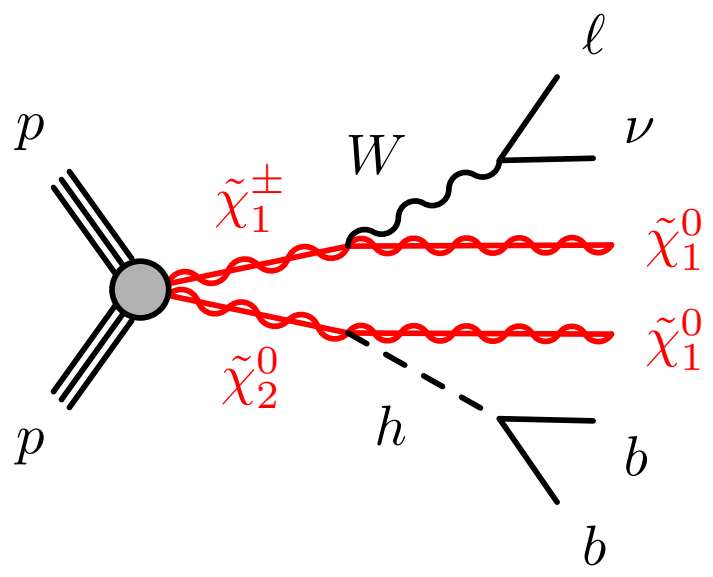

Fig. 1 A diagram illustrating the signal scenario considered for the production of a chargino and a next-to-lightest neutralino

transverse momentum $\left(\boldsymbol{p}_{\mathrm{T}}^{\text {miss }}\right)$ from neutralinos and neutrinos. A set of simplified SUSY models is used to optimise the search and interpret the results. The branching ratios of $\tilde{\chi}_{1}^{ \pm} \rightarrow W \tilde{\chi}_{1}^{0}$ and $\tilde{\chi}_{2}^{0} \rightarrow h \tilde{\chi}_{1}^{0}$ are assumed to be $100 \%$. The branching ratio of $h \rightarrow b \bar{b}$ is taken to be $58.3 \%$ as expected for the SM Higgs boson.

Previous searches for charginos and neutralinos at the LHC targeting decays via the Higgs boson have been reported by the ATLAS [25] and CMS [26] collaborations. Because of increased integrated luminosity and an improved twodimensional fit procedure, the search presented here significantly extends the SUSY parameter space sensitivity beyond that of the previously published 13 TeVATLAS search [25] for the same final state.

\section{ATLAS detector}

The ATLAS detector [27] is a multipurpose particle detector with a nearly $4 \pi$ coverage in solid angle. ${ }^{1}$ It consists of an inner tracking detector surrounded by a thin superconducting solenoid providing a $2 \mathrm{~T}$ axial magnetic field, electromagnetic and hadron calorimeters, and a muon spectrometer. The inner tracking detector covers the pseudorapidity range $|\eta|<2.5$. It consists of silicon pixel, sili-

\footnotetext{
${ }^{1}$ ATLAS uses a right-handed coordinate system with its origin at the nominal interaction point in the centre of the detector. The positive $x$ axis is defined by the direction from the interaction point to the centre of the LHC ring, with the positive $y$-axis pointing upwards, while the beam direction defines the $z$-axis. Cylindrical coordinates $(r, \phi)$ are used in the transverse plane, $\phi$ being the azimuthal angle around the $z$-axis. The pseudorapidity $\eta$ is defined in terms of the polar angle $\theta$ by $\eta=-\ln \tan (\theta / 2)$. Rapidity is defined as $y=0.5 \ln \left[\left(E+p_{z}\right) /\left(E-p_{z}\right)\right]$ where $E$ denotes the energy and $p_{z}$ is the component of the momentum along the beam direction. The angular distance $\Delta R$ is defined as $\sqrt{(\Delta y)^{2}+(\Delta \phi)^{2}}$.
}

con microstrip, and transition radiation tracking detectors. A new inner pixel layer, the insertable B-layer [28,29], was added at a mean radius of $3.3 \mathrm{~cm}$ before the start of 2015 data taking period, improving the identification of $b$-jets. Lead/liquid-argon (LAr) sampling calorimeters provide electromagnetic (EM) energy measurements with high granularity. A steel/scintillator-tile hadron calorimeter covers the central pseudorapidity range $(|\eta|<1.7)$. The endcap and forward regions are instrumented with LAr calorimeters for EM and hadronic energy measurements up to $|\eta|=4.9$. The muon spectrometer surrounds the calorimeters and is based on three large air-core toroidal superconducting magnets with eight coils each. The field integral of the toroids ranges between 2.0 and $6.0 \mathrm{Tm}$ across most of the detector. The muon spectrometer includes a system of precision tracking chambers and fast detectors for triggering. A twolevel trigger system [30] is used to select events. The firstlevel trigger is implemented in hardware and uses a subset of the detector information to keep the accepted rate below $100 \mathrm{kHz}$. This is followed by a software-based trigger that reduces the accepted event rate to $1 \mathrm{kHz}$ on average depending on the data-taking conditions.

\section{Dataset and simulated events}

The results were obtained using $139 \mathrm{fb}^{-1}$ of $p p$ LHC collision data collected between 2015 and 2018 by the ATLAS detector, with a centre-of-mass energy of $13 \mathrm{TeV}$ and a $25 \mathrm{~ns}$ proton bunch crossing interval. In 2015-2016 the average number of interactions per bunch crossing (pile-up) was $\langle\mu\rangle=20$, increasing to $\langle\mu\rangle=38$ in 2017 and to $\langle\mu\rangle=37$ in 2018. The uncertainty in the combined 2015-2018 integrated luminosity is $1.7 \%$ [31], obtained using the LUCID-2 detector [32] for the primary luminosity measurements.

Monte Carlo (MC) simulated datasets are used to model the SM backgrounds and evaluate signal selection efficiency and yields. All simulated samples were produced using the ATLAS simulation infrastructure [33] and GEANT 4 [34], or a faster simulation based on a parameterisation of the calorimeter response and GEANT 4 for the other detector systems. All simulated events were generated with a varying number of inelastic pp interactions overlaid on the hardscattering event to model the multiple proton-proton interactions in the same and nearby bunch crossings. The pileup events are generated with PYTHIA 8.186 [35] using the NNPDF2.3LO set of PDFs [36] and the A3 tune [37]. The simulated events were reconstructed with the same algorithms as those used for data.

The backgrounds considered in this analysis are: $t \bar{t}$ pair production; single-top production ( $s$-channel, $t$-channel, and associated $W t$ production); $W / Z+$ jets production; $t \bar{t}$ production with an electroweak boson $(t t V)$; Higgs boson pro- 
Table 1 Overview of MC generators used for different simulated event samples

\begin{tabular}{|c|c|c|c|c|c|}
\hline Process & Generator & Parton shower and hadronisation & Tune & PDF & Cross-section \\
\hline$t \bar{t}$ & POWHEG- BoX v2 [55-58] & PYTHIA 8.230 [35] & A14 [49] & NNPDF2.3LO [36] & NNLO+NNLL [59] \\
\hline Single top & PowHEG- Box v2 [60-62] & PYTHIA 8.230 & A14 & NNPDF2.3LO & NLO+NNLL [63] \\
\hline$W / Z+$ jets & SHERPA 2.2.1 [64] & SHERPA 2.2 .1 & SHERPA standard & NNPDF3.0NNLO & NNLO [65] \\
\hline Diboson & SHERPA $2.2 .1 \& 2.2 .2$ & SHERPA $2.2 .1 \& 2.2 .2$ & SHERPA standard & NNPDF3.0NNLO & NLO \\
\hline Triboson & SHERPA $2.2 .1 \& 2.2 .2$ & SHERPA $2.2 .1 \& 2.2 .2$ & SHERPA standard & NNPDF3.0NNLO & NLO \\
\hline$t \bar{t}+V$ & MADGRAPH5_aMC@NLO v2.3.3 & PYTHIA 8.210 & A14 & NNPDF2.3LO & NLO [66] \\
\hline tth & POWHEG- Box v2 & РYTHIA 8.230 & AZNLO [67] & CTEQ6L1 [68] & NLO [69] \\
\hline$V h$ & POWHEG- Box v2 & PYTHIA 8.212 & A14 & NNPDF2.3LO & NLO [69] \\
\hline
\end{tabular}

duction $(t t h, V h)$; and diboson $(W W, W Z, Z Z)$ and triboson $(V V V$ where $V=W, Z$ ) production. Background samples were simulated using different $\mathrm{MC}$ event generators depending on the process. All background processes were normalised to the best available theoretical calculation of their respective cross-sections. The SHERPA samples used for $W+$ jets modelling include up to two partons at NLO and four partons at LO using Comix [38] and OpenLoops [39,40] and merged with the SHERPA parton shower [41] according to the ME+PS@NLO prescription [42-45] using the set of tuned parameters developed by the SHERPA authors. The event generators, the parton shower and hadronisation routines, and the underlying-event parameter tunes and parton distribution function (PDF) sets used in simulating the SM background processes, along with the accuracy of the theoretical crosssections, are all summarised in Table 1.

For all samples showered with PYTHIA, the EvtGen v1.2.0 [46] program was used to simulate the properties of the bottom- and charm-hadron decays. Several samples produced without detector simulation were employed to estimate systematic uncertainties associated with the specific configuration of the MC generators used for the nominal SM background samples. They include variations of the renormalisation and factorisation scales, the CKKW-L [47] matching scale, as well as different PDF sets and fragmentation/hadronisation models. Details of the MC modelling uncertainties are discussed in Sect. 7.

The SUSY signal samples were generated using MADGRAPH5_aMC@NLO v2.6.2 [48] and PYTHIA 8.230 with the A14 [49] set of tuned parameters for the modelling of the parton showering (PS), hadronisation and underlying event. The matrix element (ME) calculation is performed at tree level and include the emission of up to two additional partons. The ME-PS matching is done using the CKKW-L prescription, with a matching scale set to one quarter of the chargino and next-to-lightest neutralino mass. The NNPDF2.3LO [36] PDF set was used.

Signal cross-sections are calculated at next-to-leadingorder (NLO) accuracy in the strong coupling constant, adding the resummation of soft gluon emission at next-toleading-logarithm accuracy (NLO+NLL) [50-53]. The nominal cross-section and its uncertainty are taken as the midpoint and half-width of an envelope of cross-section predictions using different PDF sets and factorisation and renormalisation scales, as described in Ref. [54]. The simplified model has two parameters, the first being the mass of the $\tilde{\chi}_{1}^{ \pm}$ and $\tilde{\chi}_{2}^{0}$ (which are assumed to be equal), and the second being the mass of the $\tilde{\chi}_{1}^{0}$. The signal cross-sections decrease as the $\tilde{\chi}_{1}^{ \pm} / \tilde{\chi}_{2}^{0}$ mass increases, ranging from $769 \mathrm{fb}$ for a $250 \mathrm{GeV}$ $\tilde{\chi}_{1}^{ \pm} / \tilde{\chi}_{2}^{0}$ mass to $1.3 \mathrm{fb}$ for a $1000 \mathrm{GeV} \tilde{\chi}_{1}^{ \pm} / \tilde{\chi}_{2}^{0}$ mass.

\section{Event reconstruction}

Events are required to have at least one reconstructed interaction vertex with a minimum of two associated tracks each having $p_{\mathrm{T}}>500 \mathrm{MeV}$. In events with multiple vertices, the one with the highest sum of squared transverse momenta of associated tracks is chosen as the primary vertex (PV) [70]. A set of baseline quality criteria are applied to reject events with non-collision backgrounds or detector noise [71].

Two identification levels are defined for leptons and jets: 'baseline' and 'signal'. Baseline leptons and jets are selected with looser identification criteria, and are used in computing the missing transverse momentum as well as in resolving possible reconstruction ambiguities. Signal leptons and jets are a subset of the baseline objects with tighter quality requirements which are used to define the search regions. Isolation criteria, defined with a list of tracking-based and calorimeter-based variables, are used to select signal leptons by discriminating against semileptonic heavy-flavour decays and jets misidentified as leptons.

Electron candidates are reconstructed from energy deposits in the electromagnetic calorimeter that are matched to charged-particle tracks in the inner detector (ID) [72]. Baseline electrons are required to satisfy $p_{\mathrm{T}}>7 \mathrm{GeV}$ and $|\eta|<2.47$. They are identified using the 'loose' operating point provided by a likelihood-based algorithm, described in 
Ref. [72]. The number of hits in the innermost pixel layer is used to discriminate between electrons and converted photons. The longitudinal impact parameter $z_{0}$ relative to the PV is required to satisfy $\left|z_{0} \sin \theta\right|<0.5 \mathrm{~mm}$. The 'tight' likelihood operating point is applied for signal electron identification and the significance of the transverse impact parameter $d_{0}$ must satisfy $\left|d_{0} / \sigma\left(d_{0}\right)\right|<5$. Signal electron candidates with $p_{\mathrm{T}}<200 \mathrm{GeV}$ are further refined using the FCLoose isolation working point, while those with larger $p_{\mathrm{T}}$ are required to pass the FCHighPtCaloOnly isolation working point, as described in Ref. [72].

Muon candidates are reconstructed from matching tracks in the ID and muon spectrometer, refined through a global fit which uses the hits from both subdetectors [73]. Baseline muons must have $p_{\mathrm{T}}>6 \mathrm{GeV}$ and $|\eta|<2.7$, and satisfy the 'medium' identification criteria. Similarly to electrons, the longitudinal impact parameter $z_{0}$ relative to the PV is required to satisfy $\left|z_{0} \sin \theta\right|<0.5 \mathrm{~mm}$. Signal muon candidates are further defined with tighter pseudorapidity and impact parameter requirements, $|\eta|<2.5$ and $\left|d_{0} / \sigma\left(d_{0}\right)\right|<$ 3 . The FCLoose isolation working point is also required for signal muons [73].

Jets are reconstructed from three-dimensional topological energy clusters in the calorimeters using the anti- $k_{t}$ algorithm [74] with a radius parameter $R=0.4$ [75]. Baseline jets are selected in the region $|\eta|<4.5$ and have $p_{\mathrm{T}}>20 \mathrm{GeV}$. To suppress jets from pile-up interactions, the jets with $|\eta|<2.8$ and $p_{\mathrm{T}}<120 \mathrm{GeV}$ are required to satisfy the 'medium' working point of the jet vertex tagger (JVT), a tagging algorithm that identifies jets originating from the PV using track information [76,77]. The selection of signal jets is further refined by requiring them to be in the region $|\eta|<2.8$ and have $p_{\mathrm{T}}>30 \mathrm{GeV}$.

Jets containing $b$-hadrons are identified as ' $b$-tagged' using the MV2c10 algorithm, a multivariate discriminant based on the track impact parameters and displaced secondary vertices [78]. These $b$-tagged jets are reconstructed in the region $|\eta|<2.5$ and have $p_{\mathrm{T}}>30 \mathrm{GeV}$. The $b$ tagging working point provides an efficiency of $77 \%$ for jets containing $b$-hadrons in simulated $t \bar{t}$ events, with rejection rates of 110 and 4.9 for light-flavour jets and jets containing $c$-hadrons, respectively [79].

To resolve the reconstruction ambiguities between electrons, muons and jets, an overlap removal procedure is applied to baseline objects. First, any electron sharing the same ID track with a muon is rejected. If it shares the same ID track with another electron, the one with lower $p_{\mathrm{T}}$ is discarded. Next, jets are rejected if they lie within $\Delta R=0.2$ of a muon or if the muon is matched to the jet through ghost association [80]. Subsequently, electrons within a cone of size $\Delta R=\min \left(0.4,0.04+10 \mathrm{GeV} / p_{\mathrm{T}}\right)$ around a jet are removed. Last, muons within a cone, defined in the same way as for electrons, around any remaining jet are removed.
The missing transverse momentum $\boldsymbol{p}_{\mathrm{T}}^{\text {miss }}$, with magnitude $E_{\mathrm{T}}^{\mathrm{miss}}$ is calculated as the negative vectorial sum of the transverse momentum of all baseline reconstructed objects (electrons, muons, jets and photons [81]) and the soft term. The soft term includes all tracks associated with the PV but not matched to any reconstructed physics object. Tracks not associated with the PV are not considered in the $E_{\mathrm{T}}^{\mathrm{miss}}$ calculation, improving the $E_{\mathrm{T}}^{\text {miss }}$ resolution by suppressing the effect of pile-up $[82,83]$.

Corrections are applied to simulated events in order to account for the trigger, particle identification, and reconstruction efficiency differences between data and simulation.

\section{Event selection}

Events are recorded with the lowest-threshold $E_{\mathrm{T}}^{\text {miss }}$ trigger available, which is fully efficient for selecting events when the offline requirement of $E_{\mathrm{T}}^{\text {miss }}>240 \mathrm{GeV}$ is applied. To target the signal events, which have a leptonically decaying $W$ boson and a Higgs boson decaying into a $b \bar{b}$ pair, events are required to have exactly one signal electron or muon (but not both) and either two or three signal jets, two of which must be $b$-tagged. The signal regions (SR) are defined using variables which suppress background contributions and increase the sensitivity for signal. These variables are based on the kinematic properties of the $b$-jets, the lepton and the missing transverse momentum, and are defined as follows:

- The invariant mass of the two $b$-jets, $m_{b \bar{b}}$, is required to be in the range $100<m_{b \bar{b}}<140 \mathrm{GeV}$, in order to preferentially select $b$-jets from the Higgs boson decays.

- The invariant mass of the lepton and the leading $b$-jet is denoted by $m\left(\ell, b_{1}\right)$. For $t \bar{t}$ or single-top (particularly the $W t$-channel) backgrounds, if the lepton and the leading $b$-jet originate from the same top-quark, the $m\left(\ell, b_{1}\right)$ distribution has an endpoint at $\sqrt{m^{2}(t)-m^{2}(W)}$. For signal events, the lepton and $b$-jet are produced from the $\tilde{\chi}_{1}^{ \pm}$and $\tilde{\chi}_{2}^{0}$ decay chains, respectively. The distribution of the invariant mass depends on the mass of the SUSY particles. For signal events with high-mass $\tilde{\chi}_{1}^{ \pm} / \tilde{\chi}_{2}^{0}$, this observable provides good discrimination against background events.

- The transverse mass, $m_{\mathrm{T}}$, is defined from the lepton transverse momentum $\boldsymbol{p}_{\mathrm{T}}^{\ell}$ and $\boldsymbol{p}_{\mathrm{T}}^{\text {miss }}$ as

$m_{\mathrm{T}}=\sqrt{2 p_{\mathrm{T}}^{\ell} E_{\mathrm{T}}^{\mathrm{miss}}\left(1-\cos \left[\Delta \phi\left(\boldsymbol{p}_{\mathrm{T}}^{\ell}, \boldsymbol{p}_{\mathrm{T}}^{\mathrm{miss}}\right)\right]\right)}$,

where $\Delta \phi\left(\boldsymbol{p}_{\mathrm{T}}^{\ell}, \boldsymbol{p}_{\mathrm{T}}^{\text {miss }}\right)$ is the azimuthal angle between $\boldsymbol{p}_{\mathrm{T}}^{\ell}$ and $\boldsymbol{p}_{\mathrm{T}}^{\mathrm{miss}}$. For $W+$ jets and semileptonic $t \bar{t}$ events, in which one on-shell $W$ boson decays leptonically, the observable has an upper endpoint at the $W$ boson mass. 
Table 2 Overview of the selection criteria for the signal regions. Each of the three 'excl.' SRs is binned in three $m_{\mathrm{CT}}$ regions for a total of nine 'excl.' bins

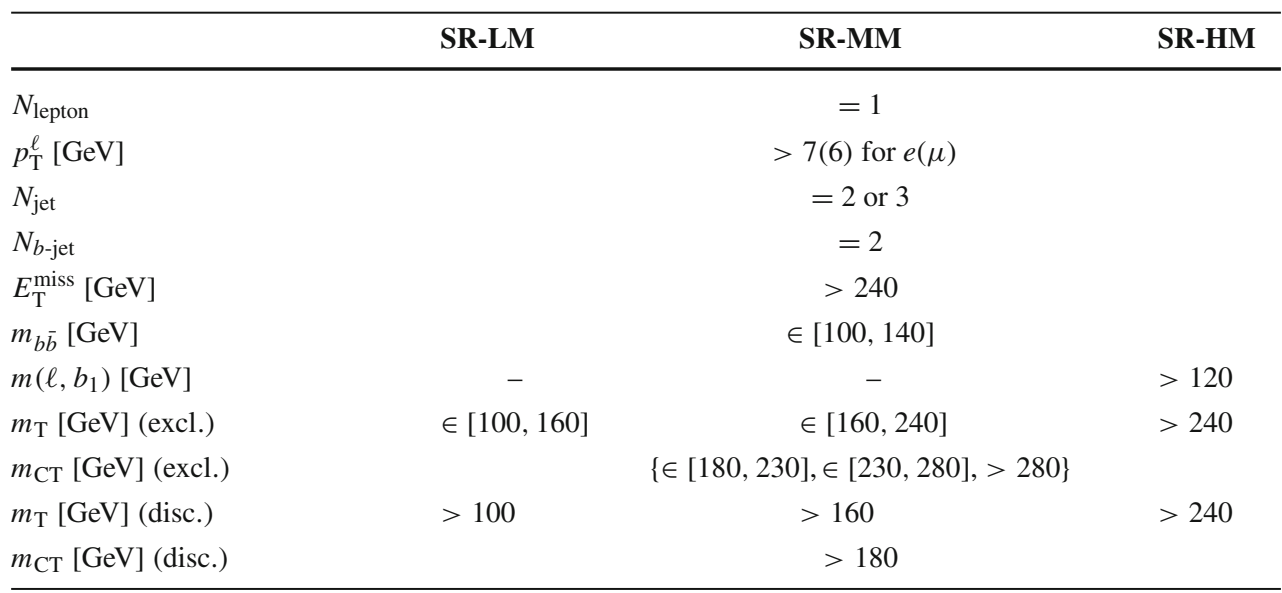

The $m_{\mathrm{T}}$ distribution for signal events extends significantly above $m(W)$.

- The contransverse mass $[84,85]$ of two $b$-jets, $m_{\mathrm{CT}}$, is defined as:

$m_{\mathrm{CT}}=\sqrt{2 p_{\mathrm{T}}^{b_{1}} p_{\mathrm{T}}^{b_{2}}\left(1+\cos \Delta \phi_{b b}\right)}$,

where $p_{\mathrm{T}}^{b_{1}}$ and $p_{\mathrm{T}}^{b_{2}}$ are the transverse momenta of the two leading $b$-jets and $\Delta \phi_{b b}$ is the azimuthal angle between them. For the $t \bar{t}$ background, the observable has an upper endpoint at $\left(m^{2}(t)-m^{2}(W)\right) / m(t)$. A requirement that $m_{\mathrm{CT}}$ be larger than $180 \mathrm{GeV}$ efficiently suppresses the $t \bar{t}$ background.

An overview of the signal region definitions is provided in Table 2. Three separate classes of signal regions are defined, progressively targeting increasing mass differences between the $\tilde{\chi}_{1}^{ \pm}$(and its mass-degenerate $\tilde{\chi}_{2}^{0}$ wino partner) and the $\tilde{\chi}_{1}^{0}$. These regions are labelled SR-LM, SR-MM and SR-HM to indicate low (LM), medium (MM) and high (HM) mass differences respectively. Requirements on $m_{\mathrm{T}}$ make the three regions mutually exclusive. Of the three signal regions, SRLM selects the smallest values of $m_{\mathrm{T}}$. It targets signal models with a mass-splitting between the $\tilde{\chi}_{2}^{0}$ (and hence the $\tilde{\chi}_{1}^{ \pm}$) and the $\tilde{\chi}_{1}^{0}$ that is similar to the Higgs boson mass. The other two signal regions select progressively larger mass differences by requiring larger values of $m_{\mathrm{T}}$. The signal region with the highest requirement on $m_{\mathrm{T}}$, SR-HM, also requires $m\left(\ell, b_{1}\right)>120 \mathrm{GeV}$ in order to further suppress $t \bar{t}$ and single-top background events. The three signal regions otherwise share a common set of selections on $E_{\mathrm{T}}^{\mathrm{miss}}, m_{b \bar{b}}$ and $m_{\mathrm{CT}}$.

When setting model-dependent exclusion limits ('excl.'), each of the three SRs is binned in three $m_{\mathrm{CT}}$ regions, thus providing nine bins in total for a simultaneous two-dimensional fit in $m_{\mathrm{CT}}$ and $m_{\mathrm{T}}$ across the three SRs. This multi-bin approach enhances the sensitivity to a range of SUSY scenarios with different properties. For model-independent limits and null-hypothesis tests ('disc.' for discovery), the various $m_{\mathrm{CT}}$ bins are merged for each of the three SRs. The requirement of $m\left(\ell, b_{1}\right)>120 \mathrm{GeV}$ is only applied in SR-HM. Furthermore, the upper bound on $m_{\mathrm{T}}$ is removed for SRLM and SR-MM. The fit strategy is detailed in Sect. 6. The systematic uncertainties, fit and results discussed in the following sections are based on the exclusion SRs, while the model-independent results are based on the discovery SRs.

\section{Background estimation}

The expected backgrounds in each signal region are determined in a profile likelihood fit, referred to as a 'backgroundonly fit'. In this fit, the normalisation of the backgrounds is adjusted to match the data in control regions with negligible signal contamination. The resulting normalisation factors are then used to correct the expected yields of the corresponding backgrounds in the various signal regions. The control regions - as detailed in the following - are designed to be enriched in the major background processes: $t \bar{t}$, singletop and $W+$ jets processes. The control region for singletop has a similar composition in single-top processes as the signal regions, therefore a single scale factor is used. All CRs are designed to be non-overlapping with the signal regions and also mutually exclusive. A probability density function is defined for each of the control regions. The inputs are the observed event yield and the predicted background yield from simulation with Poisson statistical uncertainties as well as with systematic uncertainties as nuisance parameters. The nuisance parameters are constrained by Gaussian distributions with widths corresponding to the sizes of the uncertainties. The uncertainties do not only vary the scales, but also account for bin-to-bin transitions. The systematic uncertainties are detailed in Sect. 7. The prod- 
Table 3 Overview of the CR and VR definitions. All regions partially share the same selection as the SR for all variables except $m\left(\ell, b_{1}\right)$, which is not used in the CR and VR definitions

\begin{tabular}{l|lll|lll}
\hline $\mathbf{C R}$ & TR-LM & TR-MM & TR-HM & WR & STR \\
\hline$m_{b \bar{b}}[\mathrm{GeV}]$ & & $<100$ or $>140$ & & $\in[50,80]$ & $>195$ \\
$m_{\mathrm{T}}[\mathrm{GeV}]$ & $\in[100,160]$ & $\in[160,240]$ & $>240$ & $\in[50,100]$ & $>100$ & $>180$ \\
$m_{\mathrm{CT}}[\mathrm{GeV}]$ & & $<180$ & & $>180$ & VR-offMM & VR-offHM \\
\hline $\mathbf{V R}$ & VR-onLM & VR-onMM & VR-onHM & VR-offLM & $\in[50,80] \cup[160,195]$ & $\in[50,75] \cup[165,195]$ \\
$m_{b \bar{b}}[\mathrm{GeV}]$ & & $\in[100,140]$ & & $\in[50,80] \cup[160,195]$ & $\in[160,240]$ & $>240$ \\
$m_{\mathrm{T}}[\mathrm{GeV}]$ & $\in[100,160]$ & $\in[160,240]$ & $>240$ & $\in[100,160]$ & $>180$ & \\
$m_{\mathrm{CT}}[\mathrm{GeV}]$ & & $<180$ & & & & \\
\hline
\end{tabular}

uct of all the probability density functions forms the likelihood. Normalisation and nuisance parameters are correlated in all regions participating in the fit. The likelihood is maximised by adjusting the normalisation and nuisance parameters. The extrapolation of the adjusted normalisation and nuisance parameters to the signal regions is checked in validation regions (VR), as defined below, which kinematically resemble the signal regions but are expected to have less signal. The VRs do not overlap with the CRs or SRs.

Subdominant background processes, such as $Z+$ jets, diboson and multiboson, $t \bar{t}+V, t \bar{t}+h$ and $V h$, which have no dedicated control regions, are normalised to the cross-sections indicated in Table 1. In the same way as for the dominant backgrounds, their expected yields in the SRs are subject to statistical and systematic uncertainties. Backgrounds with fake leptons such as jets misreconstructed as a lepton, and events with leptons originating from a jet produced by heavyflavour quarks or from photon conversions are estimated using a matrix method as described in Ref. [86], and found to be negligible in all regions.

The $t \bar{t}$ background estimation relies on a set of three CRs (labelled TR-LM, TR-MM, TR-HM), each with an $m_{\mathrm{T}}$ selection the same as in the SRs. In order to obtain samples enriched in $t \bar{t}$ events, the requirement on $m\left(\ell, b_{1}\right)$ is removed and the selection criteria for $m_{\mathrm{CT}}$ and $m_{b \bar{b}}$ are inverted relative to the SRs. These three control regions are fit simultaneously to obtain a single normalisation factor. The $W+$ jets contributions in the SRs are constrained by a single CR (labelled WR) defined similarly to the SRs but with less stringent lower bounds on $m_{\mathrm{T}}$ and an off-peak region for $m_{b \bar{b}}$. The fraction of events with heavy flavor hadrons in simulated $W+$ jet events was found similar between the WR and the SRs. Events in the single-top CR (labelled STR) must satisfy the SR requirements except that this CR requires $m_{\mathrm{T}}>100 \mathrm{GeV}$ and $m_{b \bar{b}}>195 \mathrm{GeV}$. The $t \bar{t}$ purity varies from $79 \%$ in TR-LM to $86 \%$ in TR-MM.
The purity of the single-top ( $W+$ jets) is $52 \%(53 \%)$ in STR (WR).

Two sets of VRs are defined for each SR, including the offpeak $\left(m_{b \bar{b}}<100\right.$ or $\left.>140 \mathrm{GeV}\right)$ and the on-peak $m_{b \bar{b}}$ regions, with the same $m_{\mathrm{T}}$ as in the SR. The on-peak VRs validate the extrapolation from the CRs to the SRs in $m_{b \bar{b}}$, and the offpeak VRs validate the extrapolation in $m_{\mathrm{CT}}$. The validation regions share the same $m_{\mathrm{T}}$ binning as the signal regions, denoted as LM, MM and HM. The background modelings are validated in low, medium and high $m_{\mathrm{T}}$ regions separately. A summary of all CR and VR selection criteria is reported in Table 3.

\section{Systematic uncertainties}

Systematic uncertainties are evaluated for all simulated signal and background events. For the dominant backgrounds with dedicated control regions, the systematic uncertainties impact the extrapolation from the control regions to the corresponding signal regions. For all other backgrounds estimated from simulation, the uncertainties affect the overall cross-section normalisation and the acceptance of the analysis selection. Uncertainties arising from theoretical modelling and detector effects are estimated and discussed below. A breakdown of the dominant systematic uncertainties in background estimates in the various exclusion signal regions is summarised in Table 4. The uncertainties in the scale factor fits to the control regions are listed as 'Normalisation of dominant backgrounds'.

Several uncertainties in the theoretical modelling of the single-top and $t \bar{t}$ backgrounds are considered. Uncertainties due to the choice of hard-scatter generation program are estimated by comparing POWHEG- BOX generated events, showered using PYTHIA 8, with events generated by aMC@NLO and showered with PYTHIA 8, while those due to the choice of parton shower model are evaluated by comparing POWHEGBoX generated samples showered using PYTHIA 8 with 
Table 4 Breakdown of the dominant systematic uncertainties in background estimates in the various exclusion signal regions. The individual uncertainties can be correlated, and do not necessarily add up in quadrature to the total background uncertainty. The percentages show the size of the uncertainty relative to the total expected background

\begin{tabular}{llll}
\hline Signal region & SR-LM & SR-MM & SR-HM \\
\hline Total background expectation & 27 & 8.6 & 8.1 \\
Total uncertainty & $\pm 4[15 \%]$ & $\pm 2.2[25 \%]$ & $\pm 2.7[34 \%]$ \\
\hline Theoretical systematic uncertainties & & & \\
\hline$t \bar{t}$ & $\pm 2.6[10 \%]$ & $\pm 0.6[7 \%]$ & $\pm 0.33[4 \%]$ \\
Single top & $\pm 0.8[2.7 \%]$ & $\pm 1.1[12 \%]$ & $\pm 1.9[23 \%]$ \\
$W+$ jets & $\pm 0.23[0.9 \%]$ & $\pm 0.07[0.8 \%]$ & $\pm 0.19[2.3 \%]$ \\
Other backgrounds & $\pm 0.13[0.5 \%]$ & $\pm 0.15[1.7 \%]$ & $\pm 0.08[1.0 \%]$ \\
\hline MC statistical uncertainties & & & \\
\hline MC statistics & $\pm 1.7[6 \%]$ & $\pm 1.1[13 \%]$ & $\pm 1.2[14 \%]$ \\
\hline Uncertainties in the background normalisation & & & \\
\hline Normalisation of dominant backgrounds & $\pm 1.3[5 \%]$ & $\pm 1.6[18 \%]$ & $\pm 1.3[16 \%]$ \\
\hline Experimental systematic uncertainties & & & \\
\hline$E_{\mathrm{T}}^{\text {miss } / J V T / p i l e-u p / t r i g g e r ~}$ & $\pm 1.8[7 \%]$ & $\pm 0.4[4 \%]$ & $\pm 0.4[5 \%]$ \\
Jet energy resolution & $\pm 1.6[6 \%]$ & $\pm 0.5[6 \%]$ & $\pm 0.4[5 \%]$ \\
$b$-tagging & $\pm 1.1[4 \%]$ & $\pm 0.29[3.4 \%]$ & $\pm 0.13[1.5 \%]$ \\
Jet energy scale & $\pm 0.9[3.2 \%]$ & $\pm 0.9[10 \%]$ & $\pm 0.29[4 \%]$ \\
Lepton uncertainties & $\pm 0.32[1.2 \%]$ & $\pm 0.09[1.0 \%]$ & $\pm 0.19[2.3 \%]$ \\
\hline
\end{tabular}

\begin{tabular}{|c|c|c|c|c|}
\hline & All $m_{\mathrm{CT}}$ bins & Low $m_{\mathrm{CT}}$ & Medium $m_{\mathrm{CT}}$ & High $m_{\mathrm{CT}}$ \\
\hline \multicolumn{5}{|l|}{ SR-LM } \\
\hline Observed & 34 & 16 & 11 & 7 \\
\hline Expected & $27 \pm 4$ & $8.8 \pm 2.8$ & $11.3 \pm 3.1$ & $7.3 \pm 1.5$ \\
\hline$t \bar{t}$ & $16.2 \pm 3.4$ & $4.4 \pm 2.2$ & $7.3 \pm 2.5$ & $4.6 \pm 1.2$ \\
\hline Single top & $2.7 \pm 1.8$ & $1.3 \pm 1.1$ & $0.9_{-0.9}^{+1.0}$ & $0.6 \pm 0.6$ \\
\hline$W+$ jets & $5.5 \pm 2.0$ & $2.0 \pm 0.9$ & $2.4 \pm 1.3$ & $1.1 \pm 0.5$ \\
\hline Di-/Multiboson & $0.67 \pm 0.19$ & $0.39 \pm 0.13$ & $0.09_{-0.09}^{+0.11}$ & $0.18 \pm 0.04$ \\
\hline Others & $2.23 \pm 0.29$ & $0.81 \pm 0.25$ & $0.64 \pm 0.15$ & $0.77 \pm 0.12$ \\
\hline \multicolumn{5}{|l|}{ SR-MM } \\
\hline Observed & 13 & 4 & 7 & 2 \\
\hline Expected & $8.6 \pm 2.2$ & $4.6 \pm 1.7$ & $2.6 \pm 1.3$ & $1.4 \pm 0.6$ \\
\hline$t \bar{t}$ & $2.7 \pm 1.4$ & $1.6 \pm 0.9$ & $0.8 \pm 0.7$ & $0.30 \pm 0.24$ \\
\hline Single top & $2.7 \pm 1.9$ & $1.6 \pm 1.5$ & $1.0_{-1.0}^{+1.1}$ & $0.15_{-0.15}^{+0.19}$ \\
\hline$W+$ jets & $1.5 \pm 0.7$ & $0.6 \pm 0.4$ & $0.3_{-0.3}^{+0.4}$ & $0.57 \pm 0.26$ \\
\hline Di-/Multiboson & $0.29 \pm 0.08$ & $0.09 \pm 0.04$ & $0.065 \pm 0.028$ & $0.14 \pm 0.06$ \\
\hline Others & $1.33 \pm 0.27$ & $0.69 \pm 0.20$ & $0.40 \pm 0.13$ & $0.24 \pm 0.09$ \\
\hline \multicolumn{5}{|l|}{ SR-HM } \\
\hline Observed & 14 & 6 & 5 & 3 \\
\hline Expected & $8.1 \pm 2.7$ & $4.1 \pm 1.9$ & $2.9 \pm 1.3$ & $1.1 \pm 0.5$ \\
\hline$t \bar{t}$ & $1.4 \pm 0.5$ & $0.8 \pm 0.4$ & $0.36 \pm 0.25$ & $0.22 \pm 0.15$ \\
\hline Single top & $2.0_{-2.0}^{+2.4}$ & $0.9_{-0.9}^{+1.5}$ & $0.9 \pm 0.9$ & $0.16_{-0.16}^{+0.26}$ \\
\hline$W+$ jets & $3.7 \pm 1.0$ & $1.9 \pm 0.8$ & $1.4 \pm 0.8$ & $0.45 \pm 0.19$ \\
\hline Di-/Multiboson & $0.21 \pm 0.06$ & $0.057 \pm 0.025$ & $0.075 \pm 0.027$ & $0.08 \pm 0.04$ \\
\hline Others & $0.74 \pm 0.16$ & $0.34 \pm 0.09$ & $0.19 \pm 0.08$ & $0.21 \pm 0.08$ \\
\hline
\end{tabular}

Table 5 Background fit results for the exclusion $\mathrm{SR}$ regions.

The errors shown are the statistical plus systematic uncertainties. Uncertainties in the fitted yields are symmetric by construction, except where the negative error is truncated at an event yield of zero 

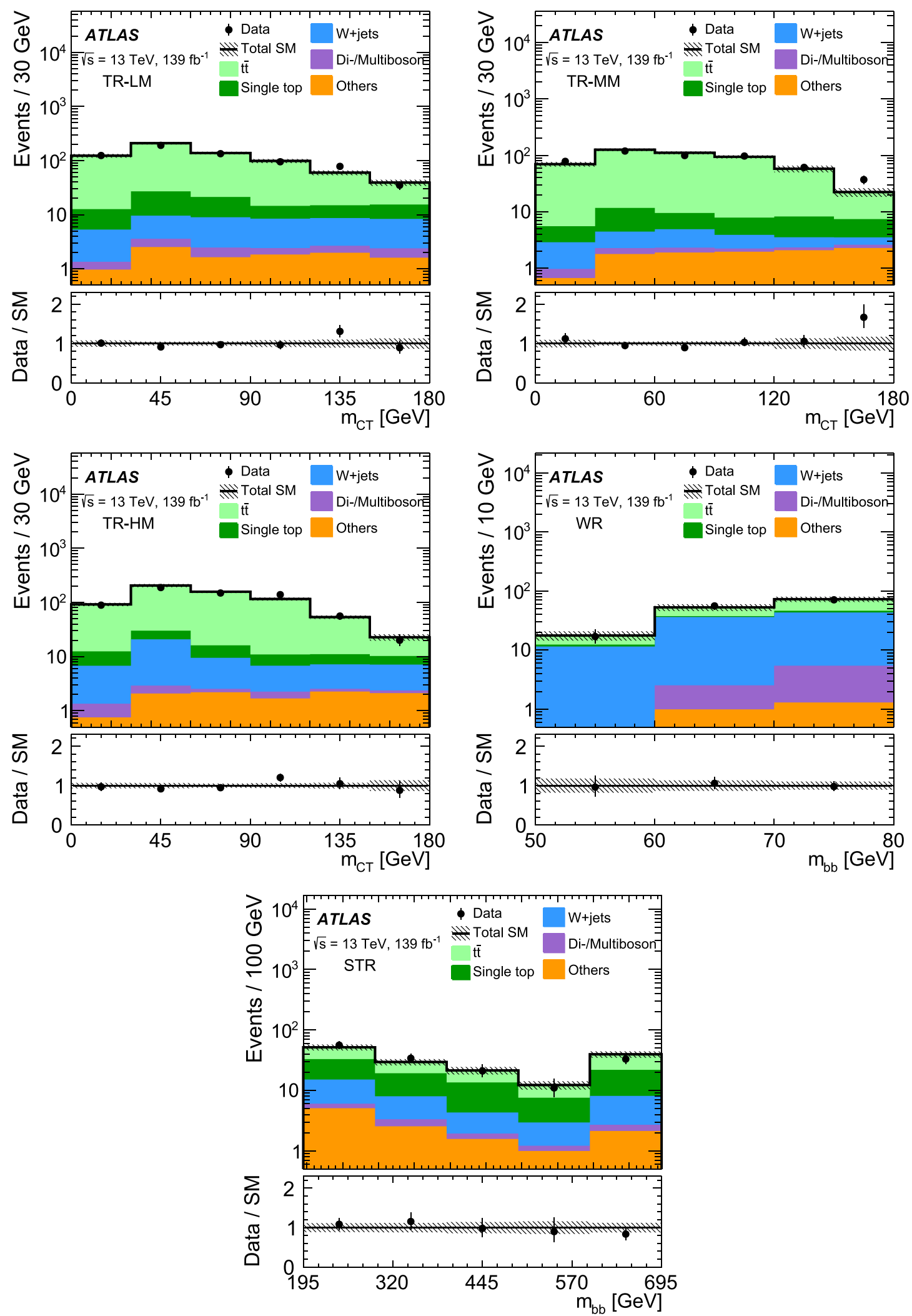

Fig. 2 The post-fit $m_{\mathrm{CT}}$ distributions in TR-LM, TR-MM, and TR-HM are shown as well as the post-fit $m_{b \bar{b}}$ distributions in WR and STR. The uncertainty bands plotted include all statistical and systematic uncertainties. The overflow events, where present, are included in the last bin 

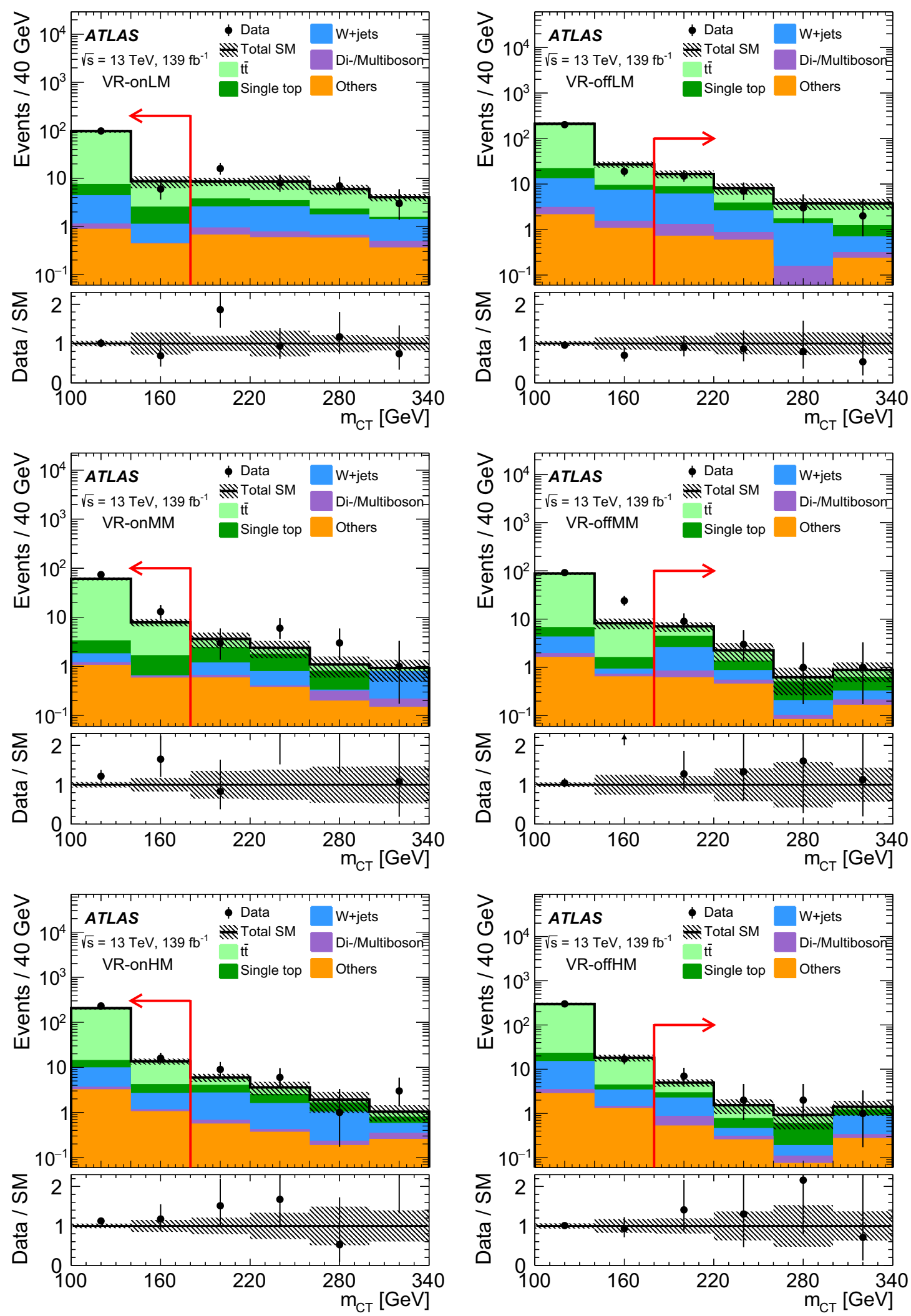

Fig. 3 The post-fit $m_{\mathrm{CT}}$ distributions are shown in each of the validation regions (VR-onLM, VR-onMM, VR-onHM, VR-offLM, VRoffMM, and VR-offHM) after all the selection requirements are applied other than the $m_{\mathrm{CT}}$ cut. The uncertainty bands plotted include all sta-

tistical and systematic uncertainties. The overflow (underflow) events, where present, are included in the last (first) bin. The line with an arrow indicates the $m_{\mathrm{CT}}$ cut used in VR selection 


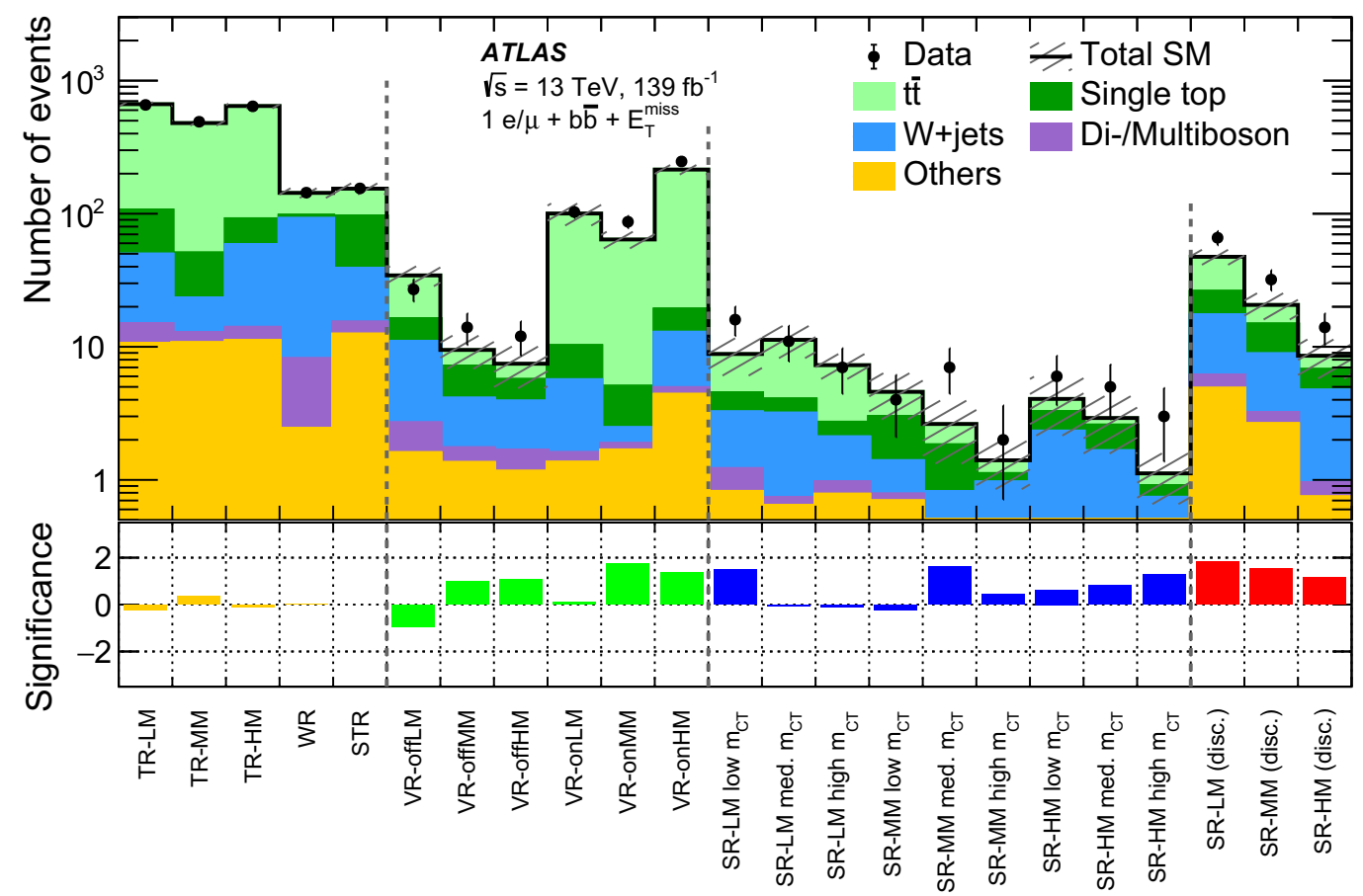

Fig. 4 Comparison of the observed and expected event yields in control, validation, exclusion, and discovery signal regions. Uncertainties in the background estimates include both the statistical (in the simulated

POWHEG- BoX samples showered using HERwIG 7 [87]. The uncertainties from the modelling of initial- and final-state radiation are assessed by varying the renormalisation and factorisation scales up and down by a factor of two, with the radiation setting varied as well [88]. For single-top $W t$ production, the impact of interference between single-resonant and double-resonant top-quark production is estimated by comparing the nominal sample generated using the diagram removal method with a sample generated using the diagram subtraction method. For the different signal regions, the dominant uncertainty sources are the $t \bar{t}$ parton shower in SR-LM $(10 \%)$, and the single-top generator uncertainties in SR-MM $(10 \%)$ and SR-HM (21\%).

Theory uncertainties affecting the generator predictions for $W / Z+$ jets, diboson, triboson and $t \bar{t}+W / Z$ samples are estimated by taking the envelope of the seven-point variations of the renormalisation and factorisation scales. For $W / Z+$ jets, the uncertainties from the PDF variations, as well as from the variations of matching and resummation scales are also considered. Additionally, an overall 6\% (20\%) systematic uncertainty in the inclusive cross-section is assigned for the diboson (triboson) sample [89]. Similar cross-section uncertainties are also assigned for other small background contributions. event yields) and systematic uncertainties. The bottom panel shows the significance [91] of the differences between the observed and expected yields. Not all regions shown here are statistically independent

Theory uncertainties in the expected yields for SUSY signals are estimated by varying by a factor of two the parameters corresponding to the factorisation, renormalisation, and CKKW-L matching scales, as well as the PYTHIA 8 shower tune parameters. The overall uncertainties range from about $10 \%$ in the region with a large splitting between the $\tilde{\chi}_{2}^{0} / \tilde{\chi}_{1}^{ \pm}$ and $\tilde{\chi}_{1}^{0}$ masses to about $25 \%$ in the mass spectra with small mass splitting.

The dominant detector systematic effects are the uncertainties associated with the jet energy scale (JES) and jet energy resolution (JER), the $E_{\mathrm{T}}^{\text {miss }}$ modelling, and pile-up. The jet uncertainties are measured as a function of the $p_{\mathrm{T}}$ and $\eta$ of the jet, the pile-up conditions and the jet flavour composition. They are determined using a combination of data and simulation, through measurements of the jet $p_{\mathrm{T}}$ balance in dijet, $Z+$ jets and $\gamma+$ jets events [90]. The systematic uncertainties in the $E_{\mathrm{T}}^{\text {miss }}$ modelling are derived by propagating the uncertainties in the energy and momentum scale of each of the objects entering the calculation, and the uncertainties in the soft term's resolution and scale [83]. A pile-up reweighting procedure is applied to simulation to match the distribution of the number of reconstructed vertices observed in data. The corresponding uncertainty is derived by a reweighting in which $\langle\mu\rangle$ is varied by $\pm 4 \%$. The experimental uncertainties have a less significant impact than the theoretical ones in all 

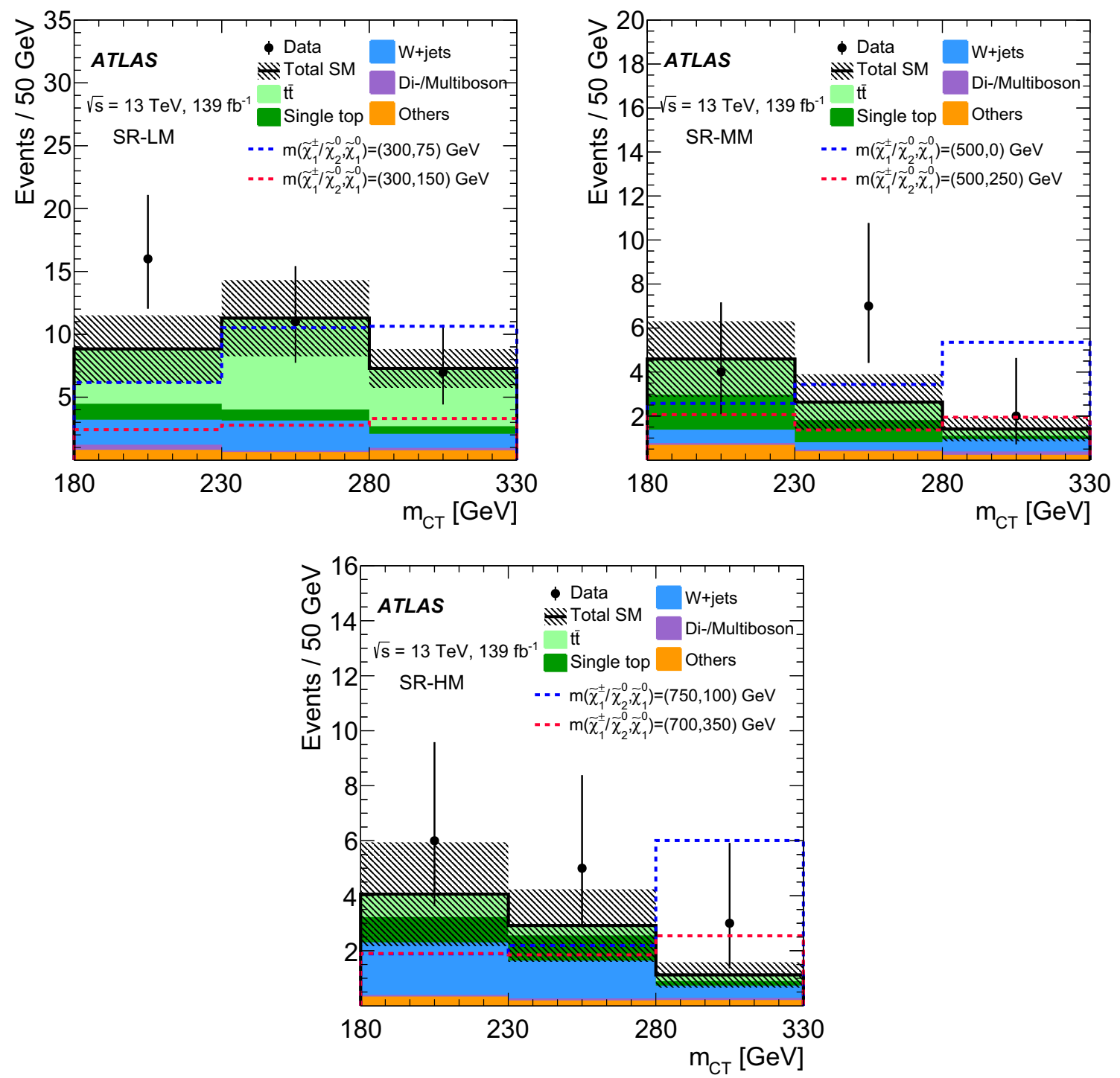

Fig. 5 The post-fit $m_{\mathrm{CT}}$ distributions in the exclusion signal regions (SR-LM, SR-MM, and SR-HM). The uncertainty bands plotted include all statistical and systematic uncertainties. The dashed lines represent the benchmark signal samples. The overflow events, where present, are included in the last bin

signal regions: the largest experimental source contributes 5$10 \%$ depending on the SR. The MC statistical uncertainties contribute $5-18 \%$ depending on the SR.

\section{Results}

The observed event yield in each of the exclusion signal regions is summarised in Table 5 along with the corresponding Standard Model predictions obtained from the background-only fit. The background normalisation factors are $1.02_{-0.09}^{+0.07}$ for $t \bar{t}, 0.6_{-0.25}^{+0.5}$ for single top, and $1.22_{-0.24}^{+0.26}$ for $W+$ jets.
In Fig. 2 the post-fit $m_{\mathrm{CT}}$ distributions in the $t \bar{t}$ control regions TR-LM, TR-MM, and TR-HM are compared with the data. For the $W$ boson and single-top control regions the $m_{b \bar{b}}$ distribution is shown. Figure 3 shows the post-fit $m_{\mathrm{CT}}$ distributions after all of the validation region selection requirements are applied except the $m_{\mathrm{CT}}$ cut. The data and the background expectation in all validation regions agree well within two standard deviations. Therefore no further systematic uncertainty is applied on the background estimation in the signal regions.

The compatibility of the observed and expected event yields in control, validation, exclusion, and discovery signal regions is illustrated in Fig. 4. No significant excess over 
Table 6 Left to right: 95\% CL upper limits on the visible cross-section $\left(\langle\epsilon \sigma\rangle_{\mathrm{obs}}^{95}\right)$ and on the number of signal events $\left(S_{\mathrm{obs}}^{95}\right)$. The third column ( $\left.S_{\exp }^{95}\right)$ shows the expected $95 \%$ CL upper limit (and its $\pm 1 \sigma$ excursions) on the number of signal events if no BSM signal is present. The last three columns indicate the $\mathrm{CL}_{\mathrm{B}}$ value, i.e. the confidence level observed for the background-only hypothesis, the discovery $p$-value $\left(p_{0}\right)$ and the significance $Z$ [91]

\begin{tabular}{lllllr}
\hline Signal Region & $\langle\epsilon \sigma\rangle_{\text {obs }}^{95}[\mathrm{fb}]$ & $S_{\text {obs }}^{95}$ & $S_{\text {exp }}^{95}$ & CL $_{\mathrm{B}}$ & $p_{0}$ \\
\hline SR-LM(disc.) & 0.26 & 36.8 & $20.0_{-5.4}^{+8.0}$ & 0.97 & 0.03 \\
SR-MM(disc.) & 0.18 & 24.8 & $15.3_{-4.6}^{+6.2}$ & 0.94 & 0.06 \\
SR-HM(disc.) & 0.11 & 14.7 & $9.7_{-2.7}^{+3.3}$ & 0.89 & 0.10 \\
\hline
\end{tabular}

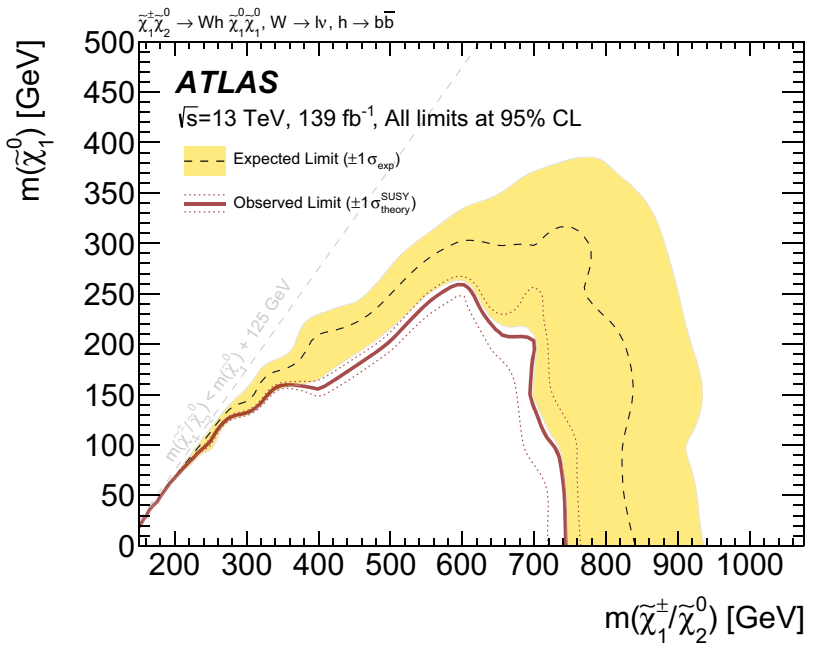

Fig. 6 Model-dependent exclusion contour at 95\% CL on the production of a chargino and a next-to-lightest neutralino. The observed limit is given by the solid line with the signal cross-section uncertainties shown by the dotted lines as indicated in the text. Expected limits are given by the dashed line with uncertainties shown by the shaded band

the SM prediction is observed in data. Figure 5 shows the post-fit $m_{\mathrm{CT}}$ distributions in SR-LM, SR-MM, and SR-HM. The uncertainty bands include all statistical and systematic uncertainties. The dashed lines represent the benchmark signal points.

Model-dependent exclusion limits at $95 \%$ confidence level (CL) are placed on the signal model. These limits are shown as a function of the masses of the supersymmetric particles in Fig. 6. They are determined interpolating between the simulated mass points, but no smoothing procedure is applied so that local fluctuations in the limit can be present. A likelihood similar to the one used in the background-only fit, but with additional terms for the SRs, is used for the calculation. The exclusion SRs thus participate in the fit and are used to constrain normalisation and nuisance parameters. A signal is allowed in this likelihood in both the CRs and SRs. The VRs are not used in the fit. The $\mathrm{CL}_{\mathrm{s}}$ method [92] is used to derive the confidence level of the exclusion for a particular signal model; signal models with a $\mathrm{CL}_{\mathrm{s}}$ value below 0.05 are excluded at $95 \% \mathrm{CL}$. The uncertainties in the observed limit are calculated by varying the cross-section for the signal up and down by its uncertainty. Due to a modest excess observed in some bins of the exclusion signal regions, the observed limit is weaker than the expected limit and extends up to about $740 \mathrm{GeV}$ in $m\left(\tilde{\chi}_{1}^{ \pm} / \tilde{\chi}_{2}^{0}\right)$ for massless $\tilde{\chi}_{1}^{0}$ and up to $m\left(\tilde{\chi}_{1}^{ \pm} / \tilde{\chi}_{2}^{0}\right)=600 \mathrm{GeV}$ for $m\left(\tilde{\chi}_{1}^{0}\right)=250 \mathrm{GeV}$. Benefiting from the increased integrated luminosity and the improved two-dimensional fit procedure, the current observed limit exceeds the previous ATLAS limit by about $200 \mathrm{GeV}$ in $m\left(\tilde{\chi}_{1}^{ \pm} / \tilde{\chi}_{2}^{0}\right)$ for a massless $\tilde{\chi}_{1}^{0}$.

Table 6 summarises the observed $\left(S_{\text {obs }}\right)$ and expected $\left(S_{\exp }\right) 95 \%$ CL upper limits on the number of signal events and on the observed visible cross-section, $\langle\epsilon \sigma\rangle_{\mathrm{obs}}^{95}$, for each of the three cumulative discovery signal regions. These cumulative signal regions are those defined to test for the presence of any beyond-the-Standard-Model (BSM) physics processes, where in every case the upper bound on $m_{\mathrm{T}}$ is also removed. Upper limits on contributions from new physics processes are estimated using the so-called 'model-independent fit', where a generic BSM process is assumed to contribute only to the SR and not to the CRs, thus giving a conservative background estimate in the SR. When normalised to the integrated luminosity of the data sample, the results can be interpreted as corresponding to observed upper limits $\langle\epsilon \sigma\rangle_{\mathrm{obs}}^{95}$, defined as the product of the production cross-section, the acceptance, and the selection efficiency of a BSM signal. The $p_{0}$ values, which represent the probability of the SM background alone to fluctuate to the observed number of events or higher, are also provided. All numbers are calculated from pseudoexperiments.

\section{Conclusion}

The results of a search for electroweakino pair production $p p \rightarrow \tilde{\chi}_{1}^{ \pm} \tilde{\chi}_{2}^{0}$ in which the chargino $\left(\tilde{\chi}_{1}^{ \pm}\right)$decays into a $W$ boson and the lightest neutralino $\left(\tilde{\chi}_{1}^{0}\right)$, while the heavier neutralino $\left(\tilde{\chi}_{2}^{0}\right)$ decays into the Standard Model $125 \mathrm{GeV}$ Higgs boson and a second $\tilde{\chi}_{1}^{0}$ are presented. The analysis is performed using $p p$ collisions provided by the LHC at a centreof-mass energy of $13 \mathrm{TeV}$. Data collected with the ATLAS detector between 2015 and 2018 are used, corresponding to 
an integrated luminosity of $139 \mathrm{fb}^{-1}$. No significant deviation from the expected Standard Model background is observed. Limits are set on the direct production of the electroweakino in simplified models. Masses of $\tilde{\chi}_{1}^{ \pm} / \tilde{\chi}_{2}^{0}$ up to $740 \mathrm{GeV}$ are excluded at $95 \%$ confidence level for a massless $\tilde{\chi}_{1}^{0}$. The current search improves on the previous ATLAS limit by about $200 \mathrm{GeV}$ in $m\left(\tilde{\chi}_{1}^{ \pm} / \tilde{\chi}_{2}^{0}\right)$ for a massless $\tilde{\chi}_{1}^{0}$.

Acknowledgements We thank CERN for the very successful operation of the LHC, as well as the support staff from our institutions without whom ATLAS could not be operated efficiently. We acknowledge the support of ANPCyT, Argentina; YerPhI, Armenia; ARC, Australia; BMWFW and FWF, Austria; ANAS, Azerbaijan; SSTC, Belarus; $\mathrm{CNPq}$ and FAPESP, Brazil; NSERC, NRC and CFI, Canada; CERN; CONICYT, Chile; CAS, MOST and NSFC, China; COLCIENCIAS, Colombia; MSMT CR, MPO CR and VSC CR, Czech Republic; DNRF and DNSRC, Denmark; IN2P3-CNRS and CEA-DRF/IRFU, France; SRNSFG, Georgia; BMBF, HGF and MPG, Germany; GSRT, Greece; RGC and Hong Kong SAR, China; ISF and Benoziyo Center, Israel; INFN, Italy; MEXT and JSPS, Japan; CNRST, Morocco; NWO, Netherlands; RCN, Norway; MNiSW and NCN, Poland; FCT, Portugal; MNE/IFA, Romania; MES of Russia and NRC KI, Russia Federation; JINR; MESTD, Serbia; MSSR, Slovakia; ARRS and MIZŠ, Slovenia; DST/NRF, South Africa; MINECO, Spain; SRC and Wallenberg Foundation, Sweden; SERI, SNSF and Cantons of Bern and Geneva, Switzerland; MOST, Taiwan; TAEK, Turkey; STFC, United Kingdom; DOE and NSF, United States of America. In addition, individual groups and members have received support from BCKDF, CANARIE, Compute Canada and CRC, Canada; ERC, ERDF, Horizon 2020, Marie Skłodowska-Curie Actions and COST, European Union; Investissements d'Avenir Labex, Investissements d'Avenir Idex and ANR, France; DFG and AvH Foundation, Germany; Herakleitos, Thales and Aristeia programmes co-financed by EU-ESF and the Greek NSRF, Greece; BSF-NSF and GIF, Israel; CERCA Programme Generalitat de Catalunya and PROMETEO Programme Generalitat Valenciana, Spain; Göran Gustafssons Stiftelse, Sweden; The Royal Society and Leverhulme Trust, United Kingdom. The crucial computing support from all WLCG partners is acknowledged gratefully, in particular from CERN, the ATLAS Tier-1 facilities at TRIUMF (Canada), NDGF (Denmark, Norway, Sweden), CC-IN2P3 (France), KIT/GridKA (Germany), INFN-CNAF (Italy), NL-T1 (Netherlands), PIC (Spain), ASGC (Taiwan), RAL (UK) and BNL (USA), the Tier-2 facilities worldwide and large non-WLCG resource providers. Major contributors of computing resources are listed in Ref. [93].

Data Availability Statement This manuscript has no associated data or the data will not be deposited. [Authors' comment: All ATLAS scientific output is published in journals, and preliminary results are made available in Conference Notes. All are openly available, without restriction on use by external parties beyond copyright law and the standard conditions agreed by CERN. Data associated with journal publications are also made available: tables and data from plots (e.g. cross section values, likelihood profiles, selection efficiencies, cross section limits, ...) are stored in appropriate repositories such as HEPDATA (http:// hepdata.cedar.ac.uk/). ATLAS also strives to make additional material related to the paper available that allows a reinterpretation of the data in the context of new theoretical models. For example, an extended encapsulation of the analysis is often provided for measurements in the framework of RIVET (http://rivet.hepforge.org/).]

Open Access This article is licensed under a Creative Commons Attribution 4.0 International License, which permits use, sharing, adaptation, distribution and reproduction in any medium or format, as long as you give appropriate credit to the original author(s) and the source, pro- vide a link to the Creative Commons licence, and indicate if changes were made. The images or other third party material in this article are included in the article's Creative Commons licence, unless indicated otherwise in a credit line to the material. If material is not included in the article's Creative Commons licence and your intended use is not permitted by statutory regulation or exceeds the permitted use, you will need to obtain permission directly from the copyright holder. To view a copy of this licence, visit http://creativecomm ons.org/licenses/by/4.0/.

Funded by $\mathrm{SCOAP}^{3}$.

\section{References}

1. ATLAS Collaboration, Observation of a new particle in the search for the Standard Model Higgs boson with the ATLAS detector at the LHC, Phys. Lett. B 716 (2012) 1, arXiv:1207.7214 [hep-ex]

2. CMS Collaboration, Observation of a new boson at a mass of 125 $\mathrm{GeV}$ with the CMS experiment at the LHC. Phys. Lett. B 716, 30 (2012). arXiv:1207.7235 [hep-ex]

3. ATLAS and CMS Collaborations, Combined measurement of the higgs boson mass in pp collisions at $\sqrt{s}=7$ and 8 TeVwith the ATLAS and CMS experiments, Phys. Rev. Lett. 114(2015) 191803, arXiv:1503.07589 [hep-ex]

4. ATLAS and CMS Collaborations, Measurements of the Higgs boson production and decay rates and constraints on its couplings from a combined ATLAS and CMS analysis of the LHC pp collision data at $\sqrt{s}=7$ and $8 \mathrm{TeV}$. JHEP 08, 045 (2016). arXiv:1606.02266 [hep-ex]

5. N. Sakai, Naturalness in supersymmetric GUTS. Z. Phys. C 11, 153 (1981)

6. S. Dimopoulos, S. Raby, F. Wilczek, Supersymmetry and the scale of unification. Phys. Rev. D 24, 1681 (1981)

7. L.E. Ibanez, G.G. Ross, Low-energy predictions in supersymmetric grand unified theories. Phys. Lett. B 105, 439 (1981)

8. S. Dimopoulos, H. Georgi, Softly broken supersymmetry and SU(5). Nucl. Phys. B 193, 150 (1981)

9. Y.A. Golfand, E.P. Likhtman, Extension of the Algebra of Poincare Group Generators and Violation of P Invariance. JETP Lett. 13, 323 (1971). http://www.jetpletters.ac.ru/ps/1584/article_ 24309.pdf [Pisma Zh. Eksp. Teor. Fiz. 13 (1971) 452]

10. D.V. Volkov, V.P. Akulov, Is the neutrino a goldstone particle? Phys. Lett. B 46, 109 (1973)

11. J. Wess, B. Zumino, Supergauge transformations in fourdimensions. Nucl. Phys. B 70, 39 (1974)

12. J. Wess, B. Zumino, Supergauge invariant extension of quantum electrodynamics. Nucl. Phys. B 78, 1 (1974)

13. S. Ferrara, B. Zumino, Supergauge invariant Yang-Mills theories. Nucl. Phys. B 79, 413 (1974)

14. A. Salam, J.A. Strathdee, Super-symmetry and non-Abelian gauges. Phys. Lett. B 51, 353 (1974)

15. G.R. Farrar, P. Fayet, Phenomenology of the production, decay, and detection of new hadronic states associated with supersymmetry. Phys. Lett. B 76, 575 (1978)

16. H. Goldberg, Constraint on the photino mass from cosmology, Phys. Rev. Lett. 50 1419, (1983), [Erratum: Phys. Rev. Lett. 103 (2009) 099905]

17. J.R. Ellis, J.S. Hagelin, D.V. Nanopoulos, K.A. Olive, M. Srednicki, Supersymmetric relics from the big bang. Nucl. Phys. B 238, 453 (1984)

18. R. Barbieri, G.F. Giudice, Upper bounds on supersymmetric particle masses. Nucl. Phys. B 306, 63 (1988)

19. B. de Carlos, J.A. Casas, One loop analysis of the electroweak breaking in supersymmetric models and the fine tuning problem. Phys. Lett. B 309, 320 (1993). arXiv:hep-ph/9303291 [hep-ph] 
20. P. Fayet, Supersymmetry and weak, electromagnetic and strong interactions. Phys. Lett. B 64, 159 (1976)

21. P. Fayet, Spontaneously broken supersymmetric theories of weak, electromagnetic and strong interactions. Phys. Lett. B 69, 489 (1977)

22. J. Gunion et al., Calculation and phenomenology of two body decays of neutralinos and charginos to W, Z, and Higgs Bosons. Int. J. Mod. Phys. A 2, 1145 (1987)

23. T. Moroi, Muon anomalous magnetic dipole moment in the minimal supersymmetric standard model, Phys. Rev. D 53, 6565, [Erratum: Phys. Rev. D 56(1997), 4424 (1996)]

24. J.L. Feng, T. Moroi, Supernatural supersymmetry: phenomenological implications of anomaly-mediated supersymmetry breaking. Phys. Rev. D 61, 095004 (2000)

25. ATLAS Collaboration, Search for chargino and neutralino production in final states with a Higgs boson and missing transverse momentum at $\sqrt{s}=13 \mathrm{TeV}$ with the ATLAS detector, Phys. Rev. D 100 (2019) 012006, arXiv:1812.09432 [hep-ex]

26. CMS Collaboration, Combined search for electroweak production of charginos and neutralinos in proton-proton collisions at $\sqrt{s}=$ 13 TeV. JHEP 03, 160 (2018). arXiv:1801.03957 [hep-ex]

27. ATLAS Collaboration, The ATLAS experiment at the CERN large Hadron Collider, JINST 3 (2008) S08003

28. ATLAS Collaboration, ATLAS Insertable B-Layer Technical Design Report, ATLAS-TDR-19, 2010, https://cds.cern.ch/record/ 1291633, ATLAS Insertable B-Layer Technical Design Report Addendum, ATLAS-TDR-19-ADD-1, 2012, https://cds.cern.ch/ record $/ 1451888$

29. B. Abbott et al., Production and integration of the ATLAS Insertable B-Layer. JINST 13, T05008 (2018). arXiv:1803.00844 [physics.ins-det]

30. ATLAS Collaboration, Performance of the ATLAS trigger system in 2015, Eur. Phys. J. C 77 (2017) 317, arXiv:1611.09661 [hep-ex]

31. ATLAS Collaboration, Luminosity determination in pp collisions at $\sqrt{s}=13 \mathrm{TeV}$ using the ATLAS detector at the LHC, ATLASCONF-2019-021, 2019, https://cds.cern.ch/record/2677054

32. G. Avoni et al., The new LUCID-2 detector for luminosity measurement and monitoring in ATLAS. JINST 13, P07017 (2018)

33. ATLAS Collaboration, The ATLAS Simulation Infrastructure, Eur. Phys. J. C 70 (2010) 823, arXiv:1005.4568 [physics.ins-det]

34. S. Agostinelli et al., GEANT4-a simulation toolkit. Nucl. Instrum. Meth. A 506, 250 (2003)

35. T. Sjöstrand, S. Mrenna, P.Z. Skands, A brief introduction to PYTHIA 8.1. Comput. Phys. Commun. 178, 852 (2008). arXiv:0710.3820 [hep-ph]

36. R.D. Ball et al., Parton distributions with LHC data. Nucl. Phys. B 867, 244 (2013). arXiv:1207.1303 [hep-ph]

37. ATLAS Collaboration, The Pythia 8 A3 tune description of ATLAS minimum bias and inelastic measurements incorporating the Donnachie-Landshoff diffractive model, ATL-PHYS-PUB-2016017, 2016, https://cds.cern.ch/record/2206965

38. T. Gleisberg, S. Höche, Comix, a new matrix element generator. JHEP 12, 039 (2008). arXiv:0808.3674 [hep-ph]

39. F. Cascioli, P. Maierhofer, S. Pozzorini, Scattering amplitudes with open loops. Phys. Rev. Lett. 108, 111601 (2012). arXiv:1111.5206 [hep-ph]

40. A. Denner, S. Dittmaier, L. Hofer, Collier: A fortran-based complex one-loop library in extended regularizations. Comput. Phys. Commun. 212, 220 (2017). arXiv: 1604.06792 [hep-ph]

41. S. Schumann, F. Krauss, A parton shower algorithm based on Catani-Seymour dipole factorisation. JHEP 03, 038 (2008). arXiv:0709.1027 [hep-ph]

42. S. Höche, F. Krauss, M. Schönherr, F. Siegert, A critical appraisal of NLO+PS matching methods. JHEP 09, 049 (2012). arXiv:1111.1220 [hep-ph]
43. S. Höche, F. Krauss, M. Schönherr, F. Siegert, QCD matrix elements + parton showers: tUhe NLO case. JHEP 04, 027 (2013). arXiv:1207.5030 [hep-ph]

44. S. Catani, F. Krauss, R. Kuhn, B.R. Webber, QCD matrix elements + parton showers. JHEP 11, 063 (2001). arXiv:hep-ph/0109231

45. S. Höche, F. Krauss, S. Schumann, F. Siegert, QCD matrix elements and truncated showers. JHEP 05, 053 (2009). arXiv:0903.1219 [hep-ph]

46. D.J. Lange, The EvtGen particle decay simulation package. Nucl. Instrum. Meth. A 462, 152 (2001)

47. L. Lönnblad, S. Prestel, Merging multi-leg NLO matrix elements with parton showers. JHEP 03, 166 (2013). arXiv:1211.7278 [hep$\mathrm{ph}]$

48. J. Alwall et al., The automated computation of tree-level and nextto-leading order differential cross sections, and their matching to parton shower simulations. JHEP 07, 079 (2014). arXiv: 1405.0301 [hep-ph]

49. ATLAS Collaboration, ATLAS Pythia 8 tunes to $7 \mathrm{TeV}$ data, ATL-PHYS-PUB-2014-021, 2014, URL:https://cds.cern.ch/ record/1966419

50. J. Debove, B. Fuks, M. Klasen, Threshold resummation for gaugino pair production at hadron colliders. Nucl. Phys. B 842, 51 (2011). arXiv:1005.2909 [hep-ph]

51. B. Fuks, M. Klasen, D.R. Lamprea, M. Rothering, Gaugino production in proton-proton collisions at a center-of-mass energy of 8 TeV. JHEP 1210, 081 (2012). arXiv:1207.2159 [hep-ph]

52. B. Fuks, M. Klasen, D.R. Lamprea, M. Rothering, Precision predictions for electroweak superpartner production at hadron colliders with Resummino. Eur. Phys. J. C 73, 2480 (2013). arXiv:1304.0790 [hep-ph]

53. J. Fiaschi, M. Klasen, Neutralino-chargino pair production at NLO+NLL with resummation-improved parton density functions for LHC Run II. Phys. Rev. D 98, 055014 (2018). arXiv:1805.11322 [hep-ph]

54. C. Borschensky et al., Squark and gluino production cross sections in pp collisions at $\sqrt{s}=13,14,33$ and 100 TeV. Eur. Phys. J. C 74, 3174 (2014). arXiv:1407.5066 [hep-ph]

55. S. Alioli, P. Nason, C. Oleari, E. Re, A general framework for implementing NLO calculations in shower Monte Carlo programs: the POWHEG BOX. JHEP 06, 043 (2010). arXiv:1002.2581 [hep$\mathrm{ph}]$

56. S. Frixione, P. Nason, C. Oleari, Matching NLO QCD computations with parton shower simulations: the POWHEG method. JHEP 11, 070 (2007). arXiv:0709.2092 [hep-ph]

57. S. Frixione, P. Nason, G. Ridolfi, A positive-weight next-toleading-order Monte Carlo for heavy flavour hadroproduction. JHEP 09, 126 (2007). arXiv:0707.3088 [hep-ph]

58. P. Nason, A new method for combining NLO QCD with shower Monte Carlo algorithms. JHEP 11, 040 (2004). arXiv:hep-ph/0409146

59. M. Czakon, A. Mitov, Top ++ : A program for the calculation of the top-pair cross-section at hadron colliders. Comput. Phys. Commun. 185, 2930 (2014). arXiv:1112.5675 [hep-ph]

60. E. Re, Single-top Wt-channel production matched with parton showers using the POWHEG method. Eur. Phys. J. C 71, 1547 (2011). arXiv:1009.2450 [hep-ph]

61. R. Frederix, E. Re, P. Torrielli, Single-top t-channel hadroproduction in the four-flavour scheme with POWHEG and aMC@NLO. JHEP 09, 130 (2012). arXiv:1207.5391 [hep-ph]

62. S. Alioli, P. Nason, C. Oleari and E. Re, NLO single-top production matched with shower in POWHEG: $s$ - and t-channel contributions, JHEP 09 (2009) 111, [Erratum: JHEP02,011(2010)], arXiv:0907.4076 [hep-ph]

63. M. Aliev et al., HATHOR - HAdronic Top and Heavy quarks crOss section calculatoR. Comput. Phys. Commun. 182, 1034 (2011). arXiv:1007.1327 [hep-ph] 
75. M. Cacciari, G.P. Salam, G. Soyez, The anti- $\mathrm{k}_{t}$ jet clustering algorithm. JHEP 04, 063 (2008). arXiv:0802.1189 [hep-ph]

76. ATLAS Collaboration, Performance of pile-up mitigation techniques for jets in pp collisions at $\sqrt{s}=8 \mathrm{TeV}$ using the ATLAS detector, Eur. Phys. J. C 76 (2016) 581, arXiv:1510.03823 [hep-ex]

77. M. Cacciari, G.P. Salam, G. Soyez, FastJet user manual. Eur. Phys. J. C 72, 1896 (2012). arXiv:1111.6097 [hep-ph]

78. ATLAS Collaboration, ATLAS b-jet identification performance and efficiency measurement with $t \bar{t}$ events in pp collisions at $\sqrt{s}=13$ TeV, Eur. Phys. J. C 79 (2019) 970, arXiv:1907.05120 [hep-ex]

79. ATLAS Collaboration, Optimisation and performance studies of the ATLAS b-tagging algorithms for the 2017-18 LHC run, ATL-PHYS-PUB-2017-013, 2017, URL:https://cds.cern.ch/ record/2273281

80. M. Cacciari, G.P. Salam, G. Soyez, The catchment area of jets. JHEP 04, 005 (2008). arXiv: 0802.1188 [hep-ph]

81. ATLAS Collaboration, Measurement of the photon identification efficiencies with the ATLAS detector using LHC Run 2 data collected in 2015 and 2016, Eur. Phys. J. C 79 (2019) 205, arXiv:1810.05087 [hep-ex]

82. ATLAS Collaboration, Performance of missing transverse momentum reconstruction with the ATLAS detector using proton-proton collisions at $\sqrt{s}=13$ TeV, Eur. Phys. J. C 78 (2018) 903, arXiv: 1802.08168 [hep-ex]

83. ATLAS Collaboration, $E_{T}^{\text {miss }}$ performance in the ATLAS detector using 2015-2016 LHC pp collisions, ATLAS-CONF-2018-023, 2018, URL:https://cds.cern.ch/record/2625233

\section{ATLAS Collaboration}

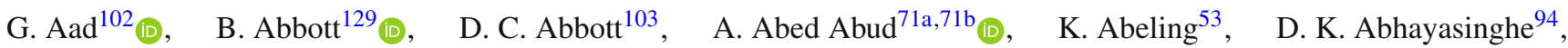

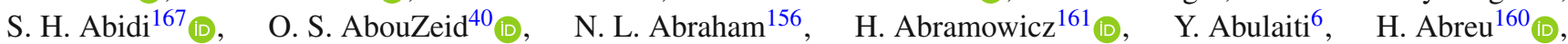

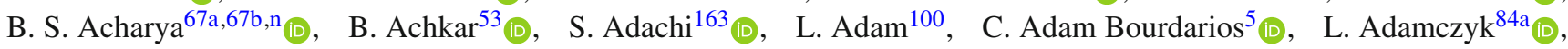

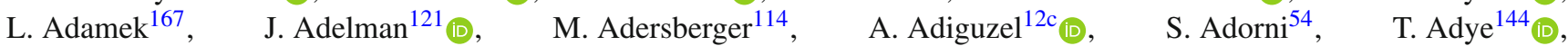
A. A. Affolder ${ }^{146}$ (D), Y. Afik ${ }^{160}$ (D), C. Agapopoulou ${ }^{65}$ (D), M. N. $\operatorname{Agaras}^{38}$ (D), A. Aggarwal ${ }^{119}$ (D), C. $\operatorname{Agheorghiesei~}^{27 c}$ (D),

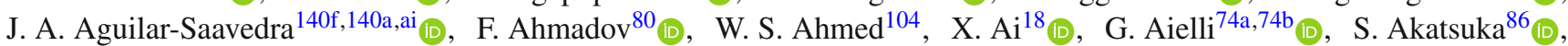

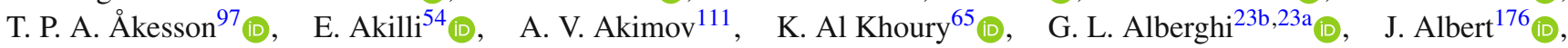

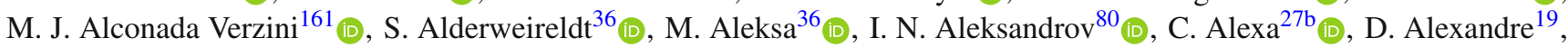

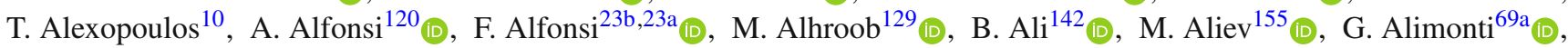

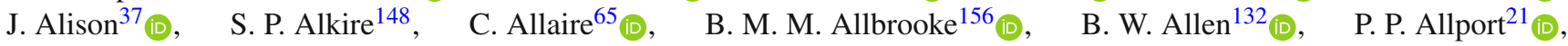

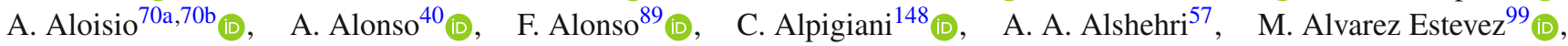

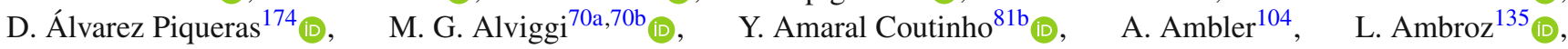

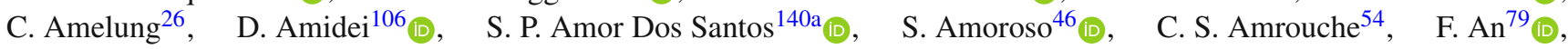

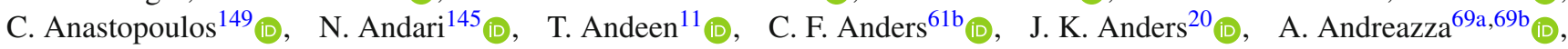

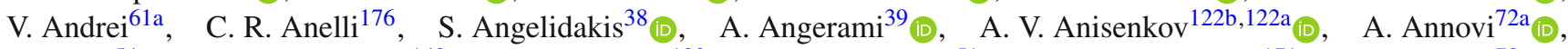

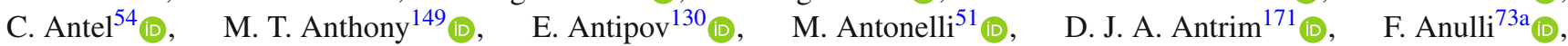

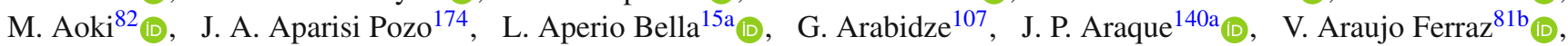

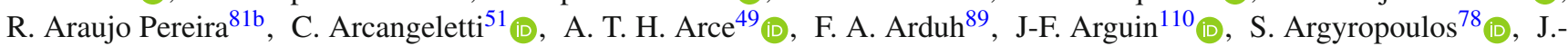

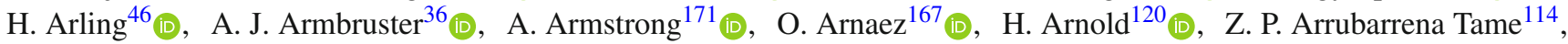

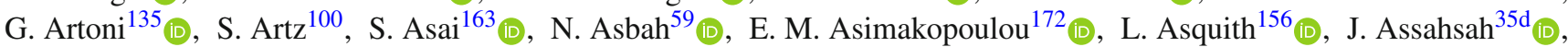

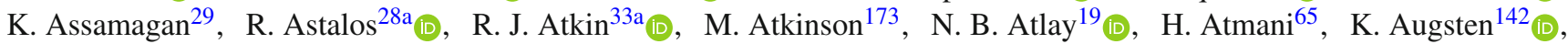

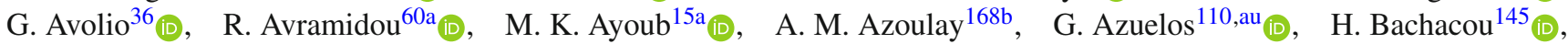

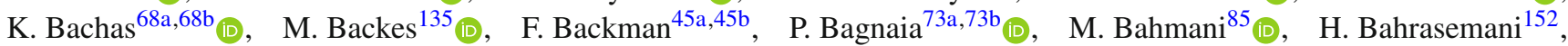
A. J. Bailey ${ }^{174}$ (D), V. R. Bailey ${ }^{173}$ (D)，J. T. Baines ${ }^{144}$ (D), M. Bajic ${ }^{40}$ (D) C. Bakalis ${ }^{10}$ ，O. K. Baker ${ }^{183}$ ， P. J. Bakker ${ }^{120}{ }^{10}$,

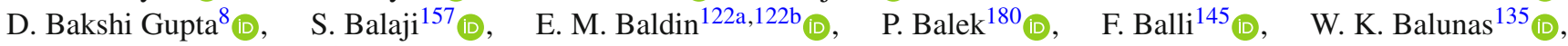

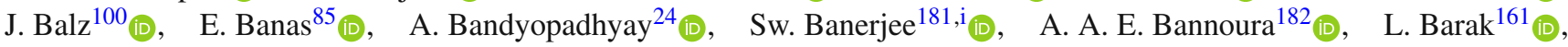




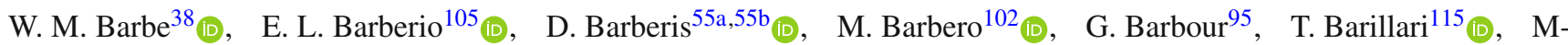
S. Barisits ${ }^{36}$ (D) J. Barkeloo ${ }^{132}$ (I) , T. Barklow ${ }^{153}$ (D), R. Barnea ${ }^{160}$, S. L. Barnes ${ }^{60 c}$, B. M. Barnett ${ }^{144}$ (D), R. M. Barnett ${ }^{18}$ (D),

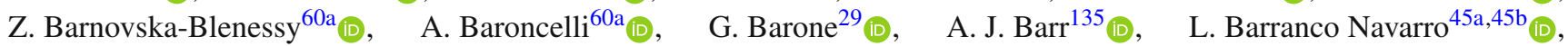

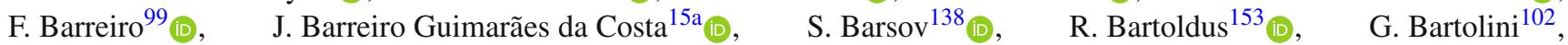

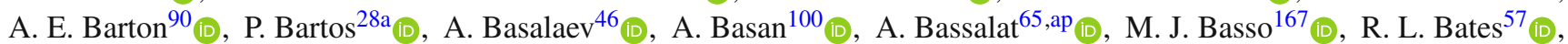

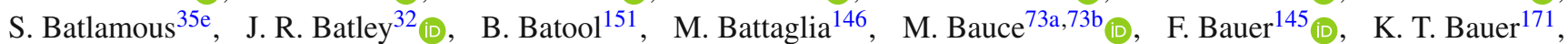

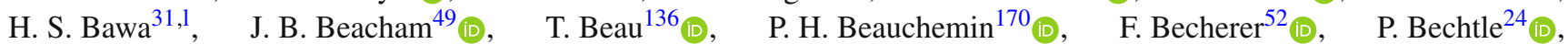

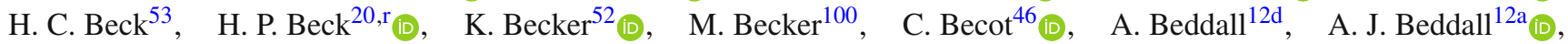
V. A. Bednyakov ${ }^{80}$ (I) M. Bedognetti ${ }^{120}$ (D) C. P. Bee ${ }^{155}$ (D) T. A. Beermann ${ }^{182}$, M. Begalli $^{81 b}$, M. Begel $^{29}$ (D) A. Behera ${ }^{155}$ (D),

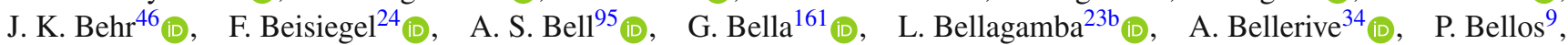

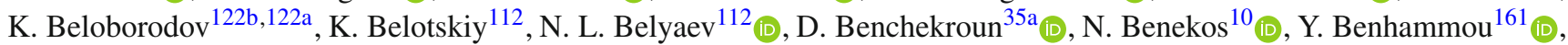
D. P. Benjamin ${ }^{6}$ (D), M. Benoit ${ }^{54}$ (D), J. R. Bensinger ${ }^{26}$ (D), S. Bentvelsen ${ }^{120}, \quad$ L. Beresford ${ }^{135}$ (D), M. Beretta ${ }^{51}$ (D),

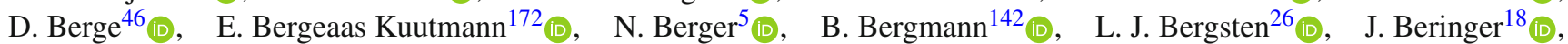

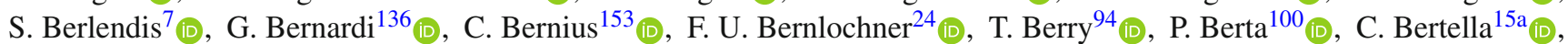

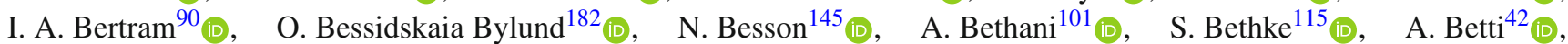

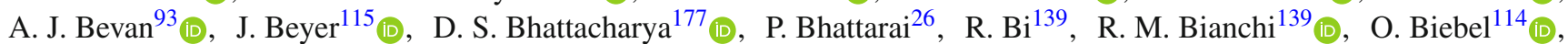

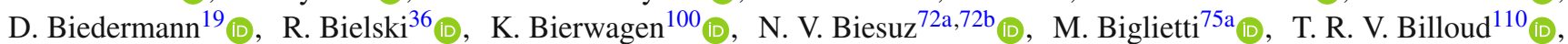

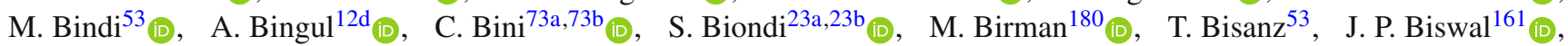

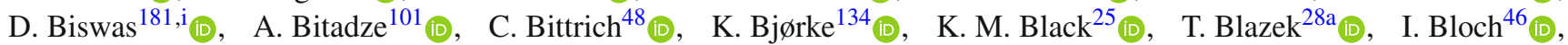

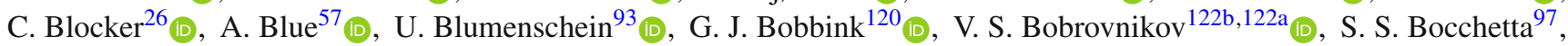

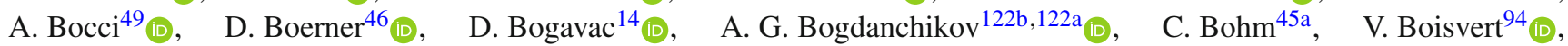

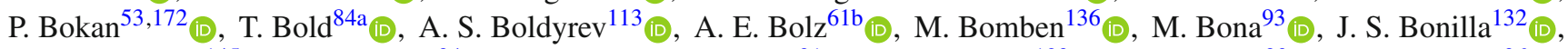
M. Boonekamp ${ }^{145}$, C. D. Booth ${ }^{94}$, H. M. Borecka-Bielska ${ }^{91}$ (D), A. Borisov ${ }^{123}$, G. Borissov ${ }^{90}$ (D), J. Bortfeldt ${ }^{36}{ }_{(\mathbb{D})}$,

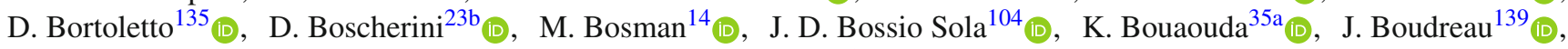

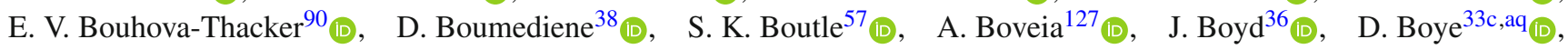

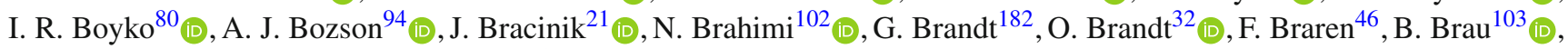

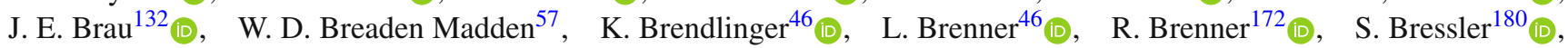
B. Brickwedde ${ }^{100}$ (D), D. L. Briglin ${ }^{21}$ (D), D. Britton ${ }^{57}$ (D) D. Britzger ${ }^{115}$ (D) I. Brock ${ }^{24}$ (D), R. Brock ${ }^{107}$, G. Brooijmans ${ }^{39}$ (D),

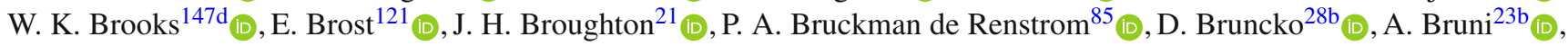

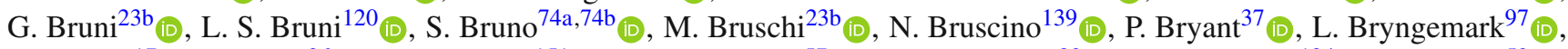

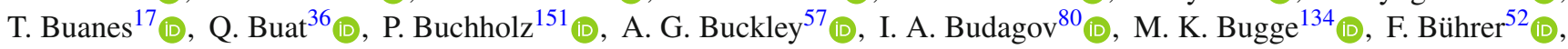

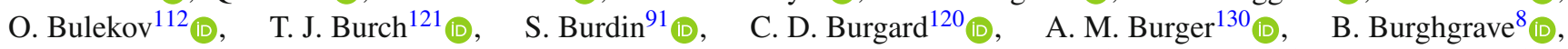
J. T. P. Burr ${ }^{46}$ (D) C. D. Burton ${ }^{11}$ (D) J. C. Burzynski ${ }^{103}$, V. Büscher ${ }^{100}$ (D), E. Buschmann ${ }^{53}$, P. J. Bussey ${ }^{57}$ (D) J. M. Butler ${ }^{25}$ (D), C. M. Buttar ${ }^{57}$ (D), J. M. Butterworth ${ }^{95}$ (D), P. Butti ${ }^{36}, \quad$ W. Buttinger ${ }^{36}$ (I),$\quad$ C. J. Buxo Vazquez ${ }^{107}, \quad$ A. Buzatu ${ }^{158}$ (D),

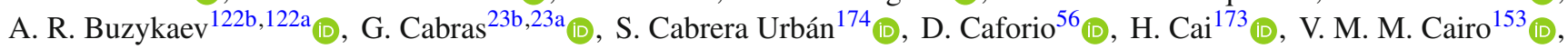

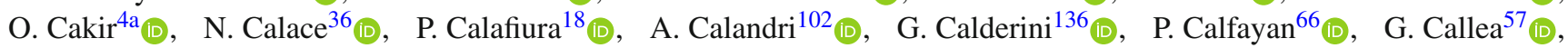
L. P. Caloba ${ }^{81 b}$, A. Caltabiano ${ }^{74 a, 74 b}, \quad$ S. Calvente Lopez $^{99}{ }^{(1)}, \quad$ D. Calvet ${ }^{38}$ (D), S. Calvet ${ }^{38}$ (D), T. P. Calvet ${ }^{155}$ (D),

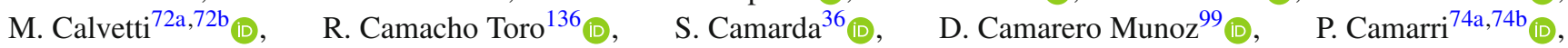
D. Cameron ${ }^{134}$ (D), R. Caminal Armadans ${ }^{103}$ (D) C. Camincher ${ }^{36}$ (D) S. Campana ${ }^{36}$, M. Campanelli ${ }^{95}$ (D), A. Camplani ${ }^{40}{ }^{(D)}$, A. Campoverde ${ }^{151}$ (D) , V. Canale ${ }^{70 a, 70 b}$ (D) A. Canesse ${ }^{104}$ (D), M. Cano Bret ${ }^{60 \mathrm{c}}$ (D), J. Cantero ${ }^{130}$ (D), T. Cao ${ }^{161}$ (D), Y. Cao ${ }^{173}$ (D),

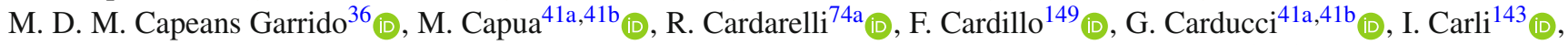

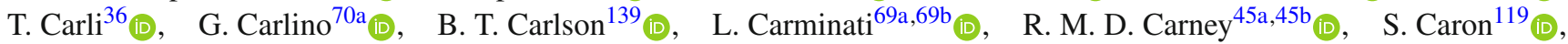

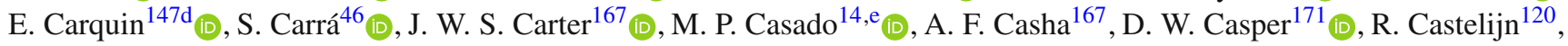

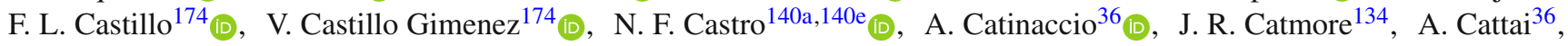

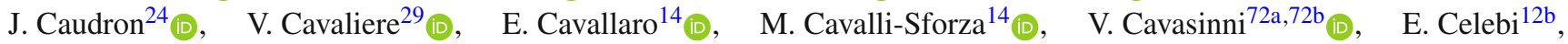

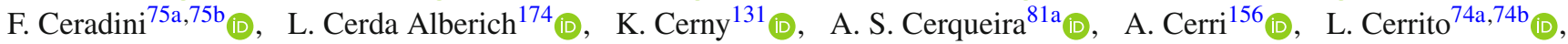

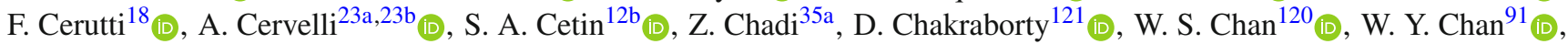
J. D. Chapman ${ }^{32}$ (D), B. Chargeishvili ${ }^{159 b}$ (D), D. G. Charlton ${ }^{21}$ (D), T. P. Charman ${ }^{93}$ (D), C. C. Chau ${ }^{34}$ (D), S. Che ${ }^{127}$ (D),

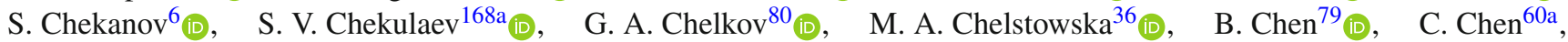
C. H. Chen ${ }^{79}$ (D) H. Hen ${ }^{29}$ (D), J. Chen ${ }^{60 a}$ (D) J. Chen $^{39}$ (D) S. Chen ${ }^{137}$ (D) S. J. Chen ${ }^{15 \mathrm{c}}$ (D) X. Chen ${ }^{15 b}$, Y. Chen ${ }^{83}$ (D), YH. Chen $^{46}$ (D) H. C. Cheng ${ }^{63}$ a (D) H. J. Cheng ${ }^{15 a}$ (D) A. Cheplakov ${ }^{80}$ (D) E. Cheremushkina ${ }^{123}$, R. Cherkaoui El Moursli ${ }^{35 e}$ (D),

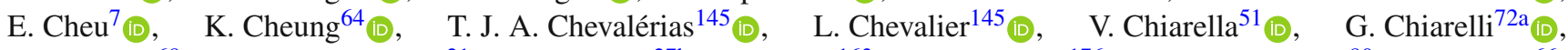

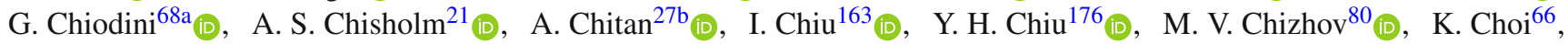




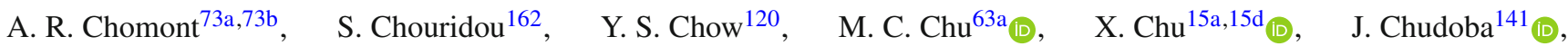
A. J. Chuinard ${ }^{104}$ (I) J. J. Chwastowski ${ }^{85}$ (D) L. Chytka ${ }^{131}$, D. Cieri ${ }^{115}$ (D), K. M. Ciesla $^{85}$ (D), D. Cinca ${ }^{47}$ (D), V. Cindro ${ }^{92}$ (D),

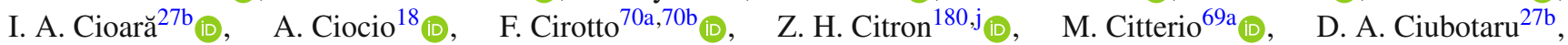

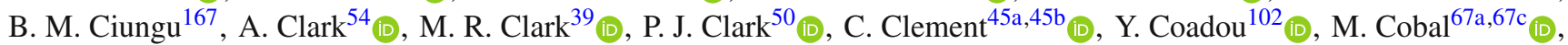
A. Coccaro ${ }^{55 b}$ (D), J. Cochran ${ }^{79}$, H. Cohen ${ }^{161}$, A. E. C. Coimbra ${ }^{36}$ (D) L. Colasurdo ${ }^{119}$ (D) B. Cole ${ }^{39}$ (D), A. P. Colijn ${ }^{120}$,

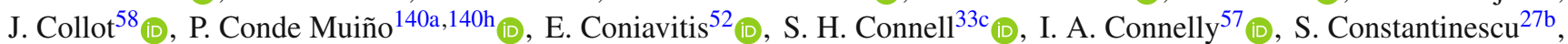
F. Conventi ${ }^{70 a, a v}(\mathbb{D})$, A. M. Cooper-Sarkar ${ }^{135}$ (D) F. Cormier ${ }^{175}$, K. J. R. Cormier ${ }^{167}$, L. D. Corpe ${ }^{95}$ (D) M. Corradi ${ }^{73 a, 73 b}{ }^{(0)}$, E. E. Corrigan ${ }^{97}$ (D), F. Corriveau ${ }^{104, a^{(D)}}$, A. Cortes-Gonzalez ${ }^{36}$ (D), M. J. Costa $^{174}$ (D), F. $\operatorname{Costanza}^{5}$ (D), D. $\operatorname{Costanzo}^{149}$ (D), G. Cowan ${ }^{94}$ (D), J. W. Cowley ${ }^{32}$ (D), J. Crane ${ }^{101}$ (D), K. Cranmer ${ }^{125}$ (D), S. J. Crawley ${ }^{57}$, R. A. Creager ${ }^{137}$ (D), S. Crépé-Renaudin ${ }^{58}$ (D), F. Crescioli ${ }^{136}{ }_{(\mathbb{D})}, \quad$ M. Cristinziani ${ }^{24}$ (D), V. Croft ${ }^{120}{ }_{(\mathbb{D})}, \quad$ G. Crosetti ${ }^{41 \mathrm{a}, 41 \mathrm{~b}}$ (D), A. Cueto ${ }^{5}$ (D), T. Cuhadar Donszelmann ${ }^{149}$ (D), A. R. Cukierman ${ }^{153}$ (D) , W. R. Cunningham ${ }^{57}$ ， S. Czekierda ${ }^{85}$ (D) , P. Czodrowski ${ }^{36}$ (D),

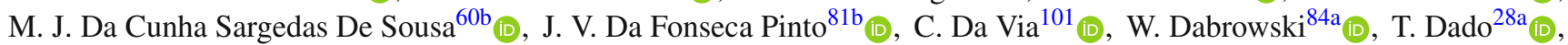

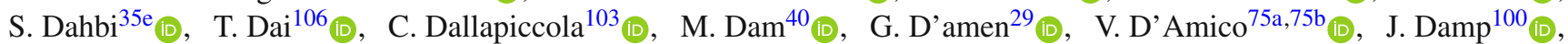

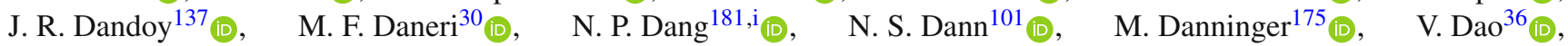

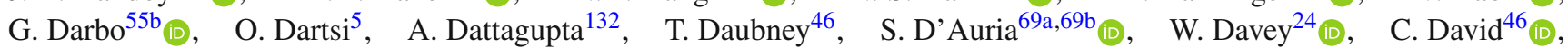
T. Davidek ${ }^{143}$ (D) D. R. Davis ${ }^{49}$ (D) I. Dawson ${ }^{149}$ (D) K. De $^{8}$, R. De Asmundis ${ }^{70 a}$ (D), M. De Beurs ${ }^{120}$, S. De Castro ${ }^{23 a}, 23 b$ (D),

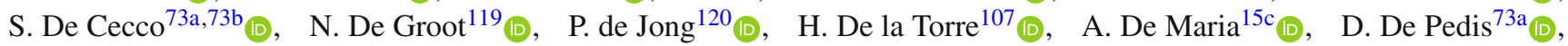

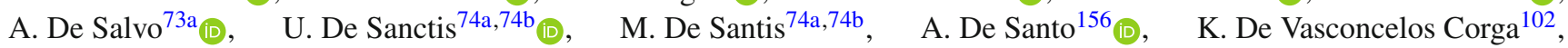

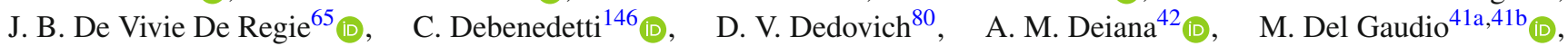

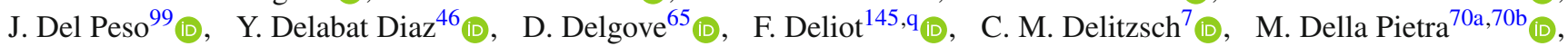

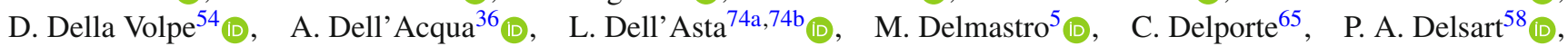
D. A. DeMarco ${ }^{167}$ (D), S. Demers ${ }^{183}$ (1) , M. Demichev $^{80}$ (D), G. Demontigny ${ }^{110}$, S. P. Denisov ${ }^{123}$, D. Denysiuk ${ }^{120}$ (D) $_{\text {, }}$

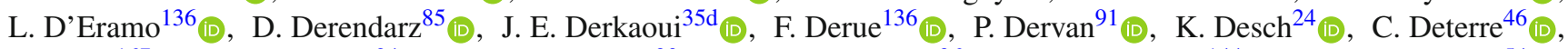

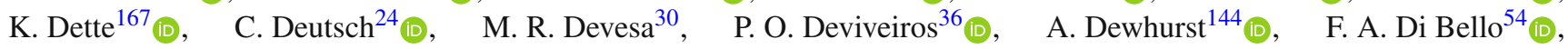

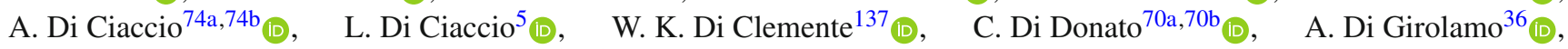

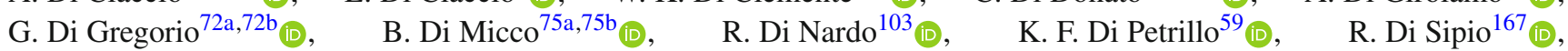
D. Di Valentino ${ }^{34}$, C. Diaconu ${ }^{102}$ (i), F. A. $\operatorname{Dias}^{40}$ (i), T. Dias Do Vale ${ }^{140 a}$ (i), M. A. Diaz ${ }^{147 a}, \quad$ J. Dickinson ${ }^{18}$ (D),

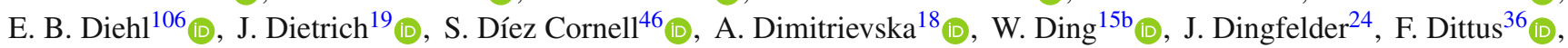

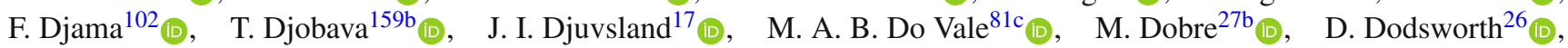

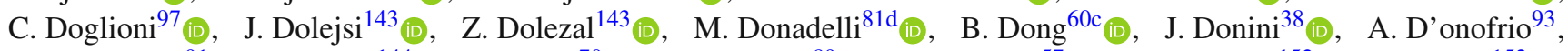
M. D’Onofrio ${ }^{91}$ (D), J. Dopke ${ }^{144}$ (D), A. Doria ${ }^{70 a}$ (D) M. T. Dova $^{89}$ (D), A. T. Doyle ${ }^{57}$ (D), E. Drechsler ${ }^{152}$ (D), E. Dreyer ${ }^{152}$ (D),

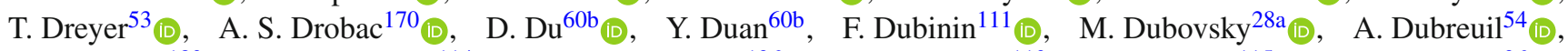

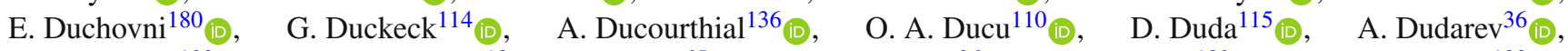

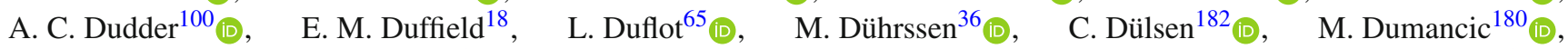

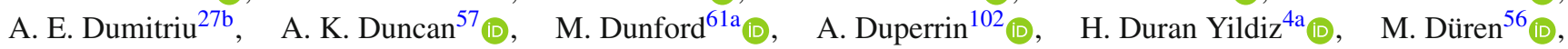
A. Durglishvili1 ${ }^{159 b}$ (D), D. Duschinger ${ }^{48}$, B. Dutta ${ }^{46}$ (D), D. Duvnjak ${ }^{1}$, G. I. Dyckes ${ }^{137}$ (D), M. Dyndal ${ }^{36}$ (D), S. Dysch ${ }^{101}$ (D), B. S. Dziedzic ${ }^{85}$ (D) K. M. Ecker $^{115}$, R. C. Edgar ${ }^{106}$, M. G. Eggleston ${ }^{49}$, T. $\operatorname{Eifert}^{36} \mathbb{D}_{\text {D }}$, G. Eigen ${ }^{17}$ (D) K. Einsweiler ${ }^{18}$ (D),

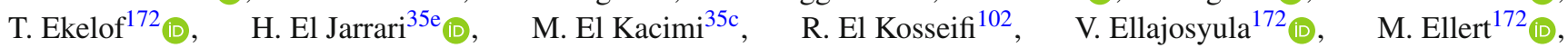

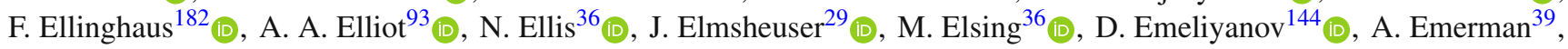
Y. Enari ${ }^{163}$ (D), M. B. Epland ${ }^{49}$ (D) J. Erdmann $^{47}$ (D), A. Ereditato ${ }^{20}$ (D) M. Errenst $^{36}$ (D) M. Escalier ${ }^{65}$ (D), C. Escobar $^{174}$ (D),

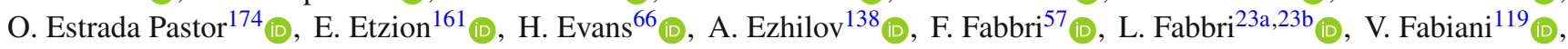
G. Facini ${ }^{95}$ (D) R. M. Faisca Rodrigues Pereira ${ }^{140} \mathrm{a}_{\mathbb{C}}$, R. M. Fakhrutdinov ${ }^{123}$, S. Falciano ${ }^{73 a}$ (D), P. J. Falke ${ }^{5}$, S. Falke ${ }^{5}$ (D),

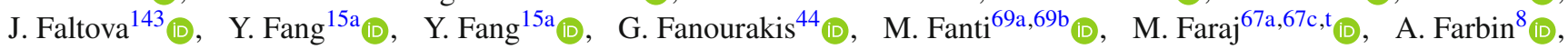

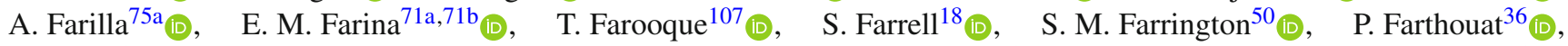

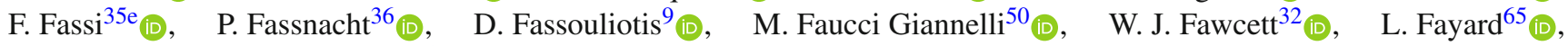
O. L. Fedin ${ }^{138, o}$, W. Fedorko ${ }^{175}$ (D), A. Fehr ${ }^{20}$ (D),$\quad$ M. Feickert ${ }^{42}$ (D) , L. Feligioni ${ }^{102}$ (D), A. Fell ${ }^{149}$ (D), C. Feng ${ }^{60 b}$ (D),

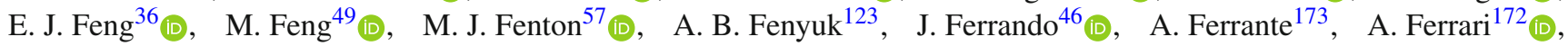

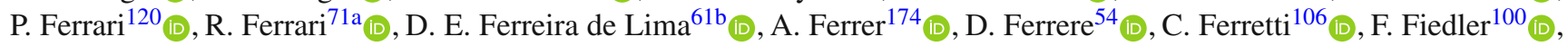

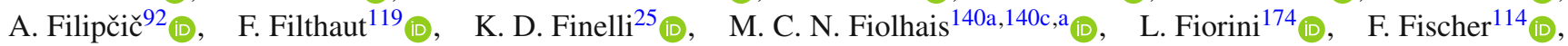
W. C. Fisher ${ }^{107}$ (D) I. Fleck ${ }^{151}$ (D), P. Fleischmann ${ }^{106}$ (D), R. R. M. Fletcher ${ }^{137}$, T. Flick ${ }^{182}$ (D), B. M. Flierl $1^{114}$ (D) , L. Flores ${ }^{137}$ (D), L. R. Flores Castillo ${ }^{63 a}$ (D) F. M. Follega ${ }^{76 a, 76 b}$ (I) N. Fomin ${ }^{17}$ (D), J. H. Foo ${ }^{167}$ (D), G. T. Forcolin ${ }^{76 a, 76 b}$ (I) , A. Formica ${ }^{145}$ (D),

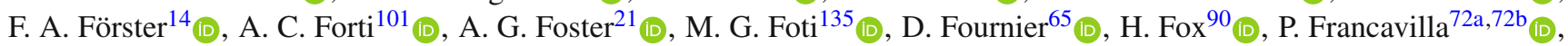

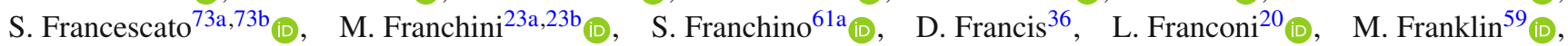




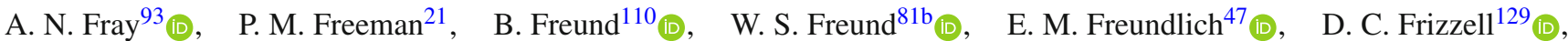

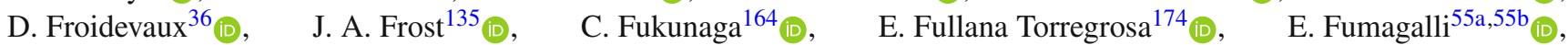

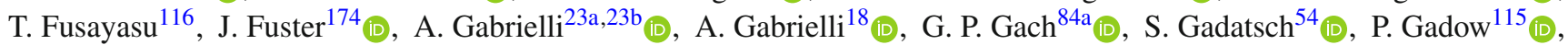

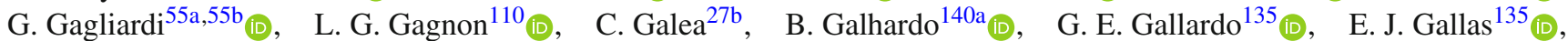
B. J. Gallop ${ }^{144}$ (D), G. Galster ${ }^{40}$, R. Gamboa Goni ${ }^{93}$ (D), K. K. Gan ${ }^{127}$ (D), S. Ganguly ${ }^{180}$ (D), J. Gao ${ }^{60 a}$ (D), Y. Gao ${ }^{50}$ (D),

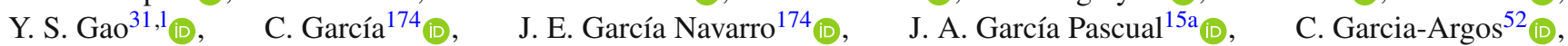

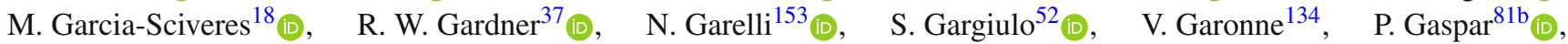

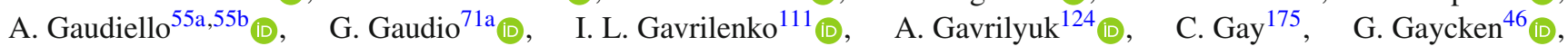

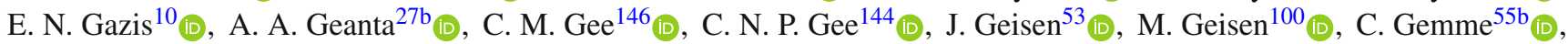
M. H. Genest ${ }^{58}$ (D) , C. Geng ${ }^{106}$, S. Gentile ${ }^{73 a, 73 b}$ (D), S. George ${ }^{94}$ (I) T. Geralis ${ }^{44}$ (D) L. O. Gerlach ${ }^{53}$, P. Gessinger-Befurt ${ }^{100}$ (D), G. Gessner ${ }^{47}$ (1), S. Ghasemi ${ }^{151}$ (D), M. Ghasemi Bostanabad ${ }^{176}$ (D), M. Ghneimat ${ }^{151}$ (D), A. Ghosh ${ }^{65}$ (D), A. Ghosh ${ }^{78}$ (D),

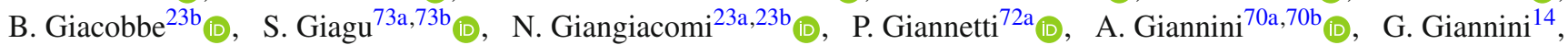

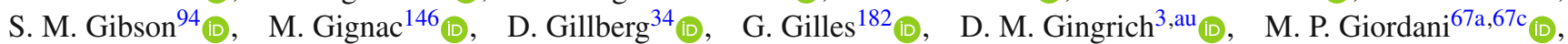

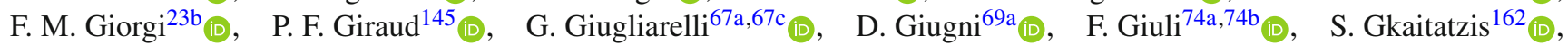

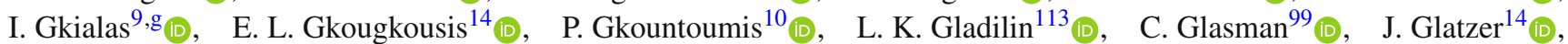
P. C. F. Glaysher ${ }^{46}$ (D), A. Glazov ${ }^{46}, \quad$ G. R. Gledhill ${ }^{132}$ (D),$\quad$ M. Goblirsch-Kolb ${ }^{26}$ (D), D. Godin ${ }^{110}, \quad$ S. Goldfarb ${ }^{105}$ (D), T. Golling ${ }^{54}(\mathbb{D})$, D. Golubkov ${ }^{123}$ (D), A. Gomes ${ }^{140 a, 140 b}{ }_{(D)}$, R. Goncalves Gama ${ }^{53}$ (D), R. Gonçalo ${ }^{140 a}$ (D), G. Gonella ${ }^{52}$ (D), L. Gonella ${ }^{21}$ (i) , A. Gongadze ${ }^{80}$ (i) F. Gonnella ${ }^{21}$ (D) J. L. Gonski ${ }^{59}$ (i) , S. González de la Hoz $^{174}$ (I) , S. Gonzalez-Sevilla ${ }^{54}$ (D),

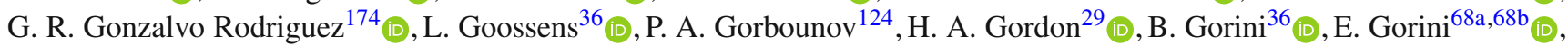

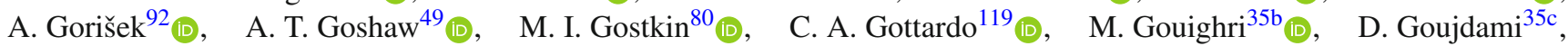

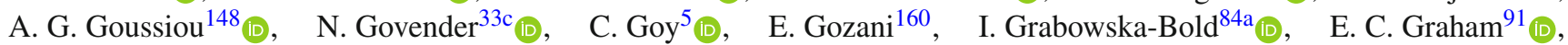

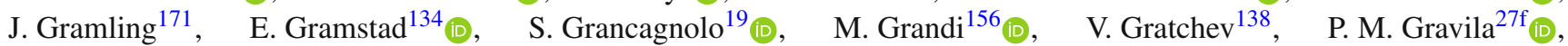
F. G. Gravili68a,68b (D) C. Gray ${ }^{57}$ (D) H. M. Gray ${ }^{18}$ (D) C. Grefe ${ }^{24}$ (D) K. Gregersen ${ }^{97}$ (D), I. M. Gregor ${ }^{46}$ (D), P. Grenier ${ }^{153}$ (D),

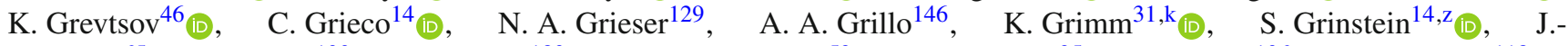
F. Grivaz ${ }^{65}$ (D), S. Groh ${ }^{100}{ }_{(D)}$, E. Gross ${ }^{180}$, J. Grosse-Knetter ${ }^{53}$ (D), Z. J. Grout ${ }^{95}$ (D), C. Grud ${ }^{106}, \quad$ A. Grummer ${ }^{118}{ }_{(\text {D }}$, L. Guan ${ }^{106}$ (D), W. Guan ${ }^{181}$ (D), C. Gubbels ${ }^{175}$ (D), J. Guenther ${ }^{36}$ (D), A. Guerguichon ${ }^{65}$ (D), J. G. R. Guerrero Rojas ${ }^{174}$ (D),

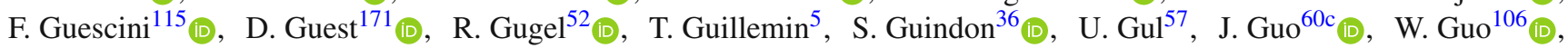

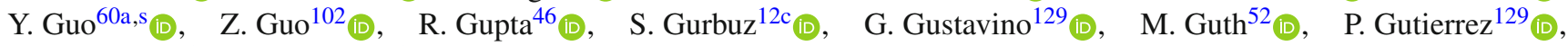
C. Gutschow ${ }^{95}$ (D) C. Guyot ${ }^{145}$, C. Gwenlan ${ }^{135}$ (D), C. B. Gwilliam ${ }^{91}$ (D) A. Haas ${ }^{125}$ (D), C. Haber ${ }^{18}$ (D) H. K. Hadavand ${ }^{8}$,

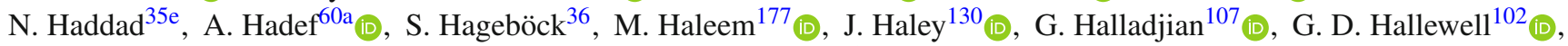

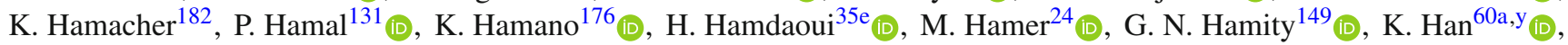

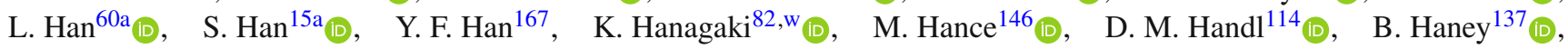

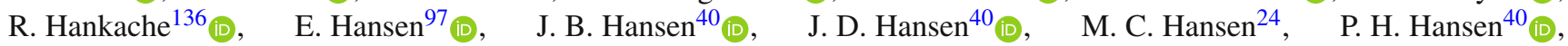
E. C. Hanson ${ }^{101}$ (D), K. Hara ${ }^{169}$ (D), T. Harenberg ${ }^{182}$ (D), S. Harkusha ${ }^{108}$ (D), P. F. Harrison ${ }^{178,}$, N. M. Hartmann ${ }^{114}$ (D),

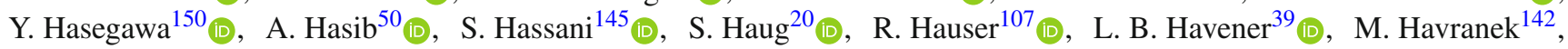

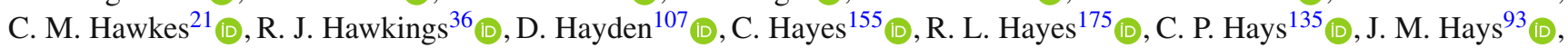

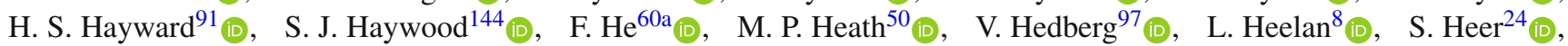

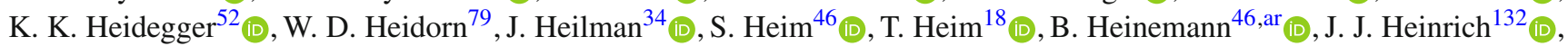

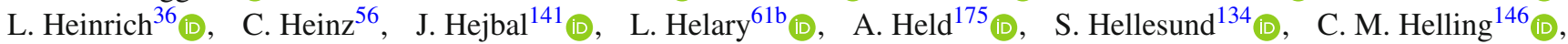

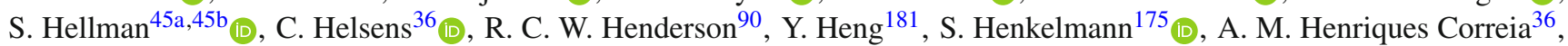
G. H. Herbert ${ }^{19}$, H. Herde ${ }^{26}$ (D) V. Herget ${ }^{177}$ (D), Y. Hernández Jiménez ${ }^{33 e}$, H. Herr ${ }^{100}$, M. G. Herrmann ${ }^{114}$ (1) , T. Herrmann ${ }^{48}$,

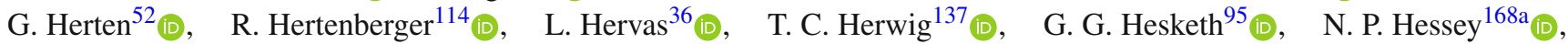

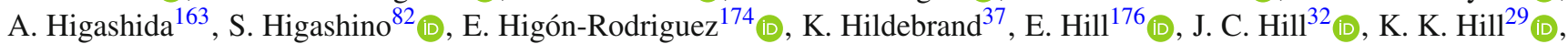

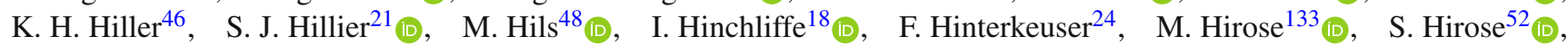

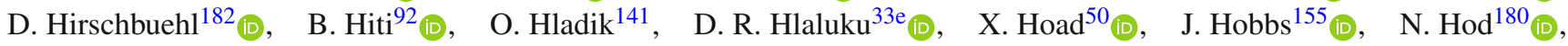
M. C. Hodgkinson ${ }^{149}$ (D), A. Hoecker ${ }^{36}$ (D) F. Hoenig ${ }^{114}$, D. Hohn ${ }^{52}$ (D) D. Hohov ${ }^{65}$, T. Holm ${ }^{24}$ (D) T. R. Holmes ${ }^{37}$ (D), M. Holzbock ${ }^{114}$ (D), L. B. A. H. Hommels ${ }^{32}$ (D), S. Honda ${ }^{169}$ (D), T. M. Hong ${ }^{139}$ (D), J. C. Honig ${ }^{52}$ (D), A. Hönle ${ }^{115}$ (D),

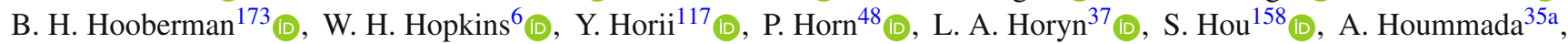
J. Howarth ${ }^{101}$ (D), J. Hoya ${ }^{89}$ (D), M. Hrabovsky ${ }^{131}$ (D), J. Hrdinka ${ }^{77}$, I. Hristova ${ }^{19}$ (D), J. Hrivnac ${ }^{65}$, A. Hrynevich ${ }^{109}$ (D),

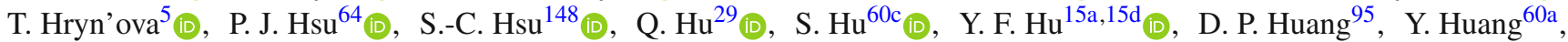

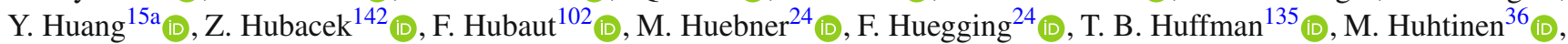
R. F. H. Hunter ${ }^{34}$ (i), P. Huo ${ }^{155}$, A. M. Hupe ${ }^{34}$, N. Huseynov ${ }^{80, a g}$, J. Huston ${ }^{107}$ (D), J. Huth ${ }^{59}$ (D), R. Hyneman ${ }^{106}$ (D), $_{\text {, }}$

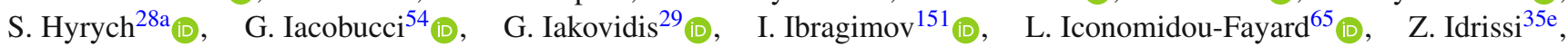




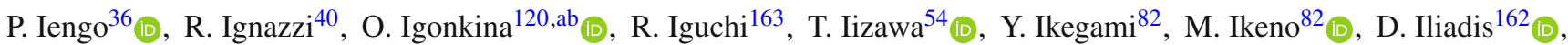

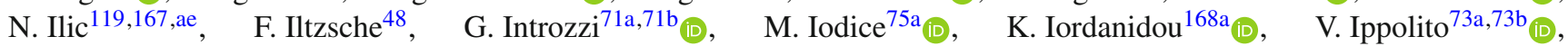

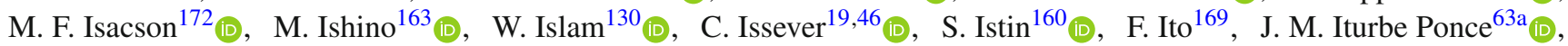

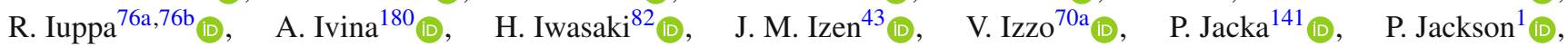

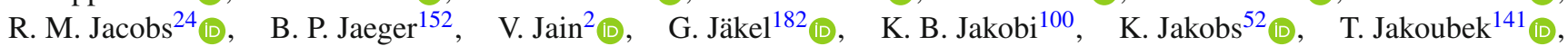
J. Jamieson ${ }^{57}$ (D), K. W. Janas ${ }^{84 a}$, R. Jansky ${ }^{54}$ (D), J. Janssen ${ }^{24}$ (D), M. Janus ${ }^{53}$ (D), P. A. Janus ${ }^{84 a}$ (D), G. Jarlskog ${ }^{97}$ (D),

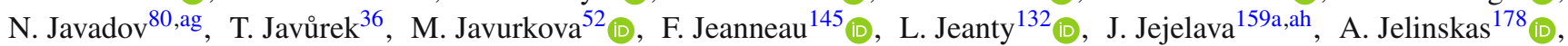

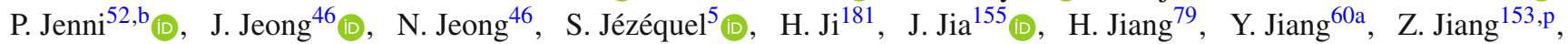

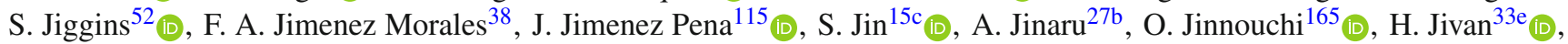

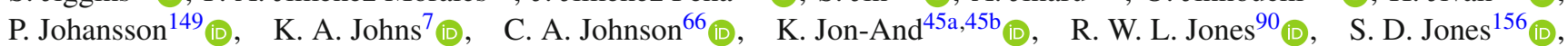

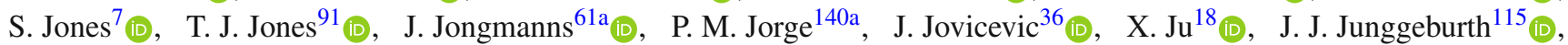
A. Juste Rozas ${ }^{14,2}$ (D), A. Kaczmarska ${ }^{85}$ (D), M. Kado ${ }^{73 a, 73 b}$, H. Kagan ${ }^{127}$, M. Kagan ${ }^{153}$ (D), A. Kahn ${ }^{39}$, C. Kahra ${ }^{100}$ (D),

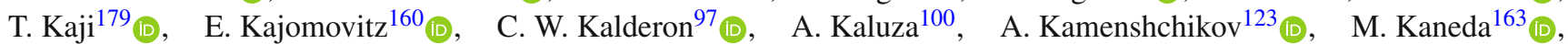

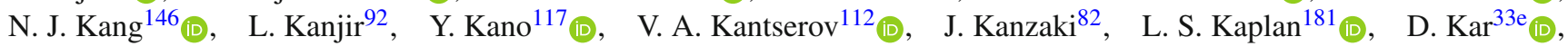

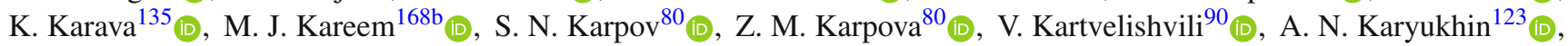

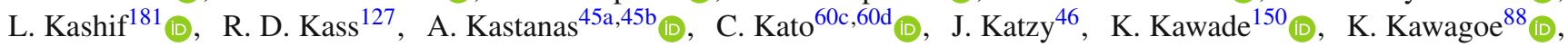

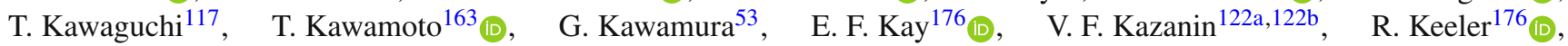

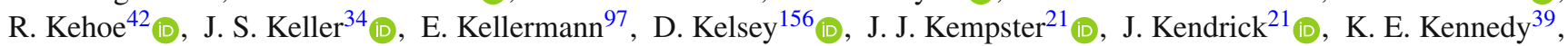
O. Kepka ${ }^{141}$ (1), S. Kersten ${ }^{182}$, B. P. Kerševan ${ }^{92}$ (D), S. Ketabchi Haghighat ${ }^{167}$ (i), M. Khader ${ }^{173}$ (i), F. Khalil-Zada ${ }^{13}$,

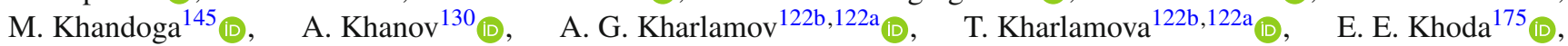

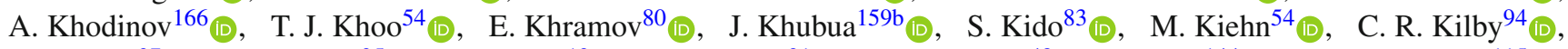
Y. K. $\operatorname{Kim}^{37}$ (D), N. Kimura ${ }^{95}$, O. M. Kind ${ }^{19}$ (D), B. T. King ${ }^{91}$, D. $\operatorname{Kirchmeier}^{48}$ (D), J. Kirk ${ }^{144}$ (D), A. E. Kiryunin ${ }^{115}$ (D), T. Kishimoto ${ }^{163}$ (D), D. P. Kisliuk ${ }^{167}$ ， V. Kitali ${ }^{46}$ (D), O. Kivernyk ${ }^{5}$ (D), T. Klapdor-Kleingrothaus ${ }^{52}$ (D), M. Klassen ${ }^{61 \mathrm{a}}$ (D), M. H. Klein ${ }^{106}{ }_{(\mathbb{D})}$, M. Klein $^{91}$ (D), U. Klein ${ }^{91}$ (D), K. Kleinknecht ${ }^{100}$, P. Klimek ${ }^{121}$ (D), A. Klimentov ${ }^{29}$ (D), T. Klingl ${ }^{24}$ (D),

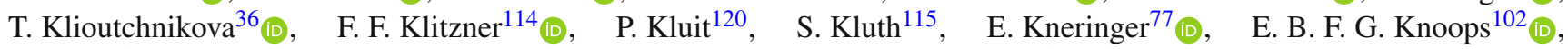
A. Knue ${ }^{52}$ (D), D. Kobayashi ${ }^{88}$, T. Kobayashi ${ }^{163}, \quad$ M. Kobel ${ }^{48}$ (D), M. Kocian ${ }^{153}$ (D), P. Kodys ${ }^{143}$ (D), P. T. Koenig ${ }^{24}$ (D),

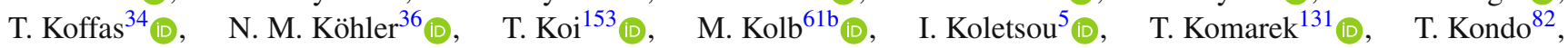
N. Kondrashova ${ }^{60 c}$, K. Köneke ${ }^{52}$ (D), A. C. König ${ }^{119}$ (D), T. Kono ${ }^{126}$ (D), R. Konoplich ${ }^{125, \text { am }}$ (D), V. Konstantinides ${ }^{95}$, N. Konstantinidis ${ }^{95}$ (D) B. Konya ${ }^{97}$ (D) R. Kopeliansky ${ }^{66}$ (D) S. Koperny ${ }^{84 a}$ (D) K. Korcy ${ }^{85}$ (D) K. Kordas ${ }^{162}$ (D) G. Koren ${ }^{161}$, A. Korn ${ }^{95}$ (D), I. Korolkov ${ }^{14}$ (D), E. V. Korolkova ${ }^{149}$, N. Korotkova ${ }^{113}$, O. Kortner ${ }^{115}$ (D), S. Kortner ${ }^{115}$ (D), T. Kosek ${ }^{143}$ (D),

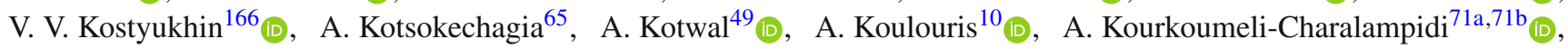
C. Kourkoumelis ${ }^{9}$ (D) E. Kourlitis ${ }^{149}$ (D), V. Kouskoura ${ }^{29}$ (D), A. B. Kowalewska ${ }^{85}$ (D), R. Kowalewski176 (D) C. Kozakai ${ }^{163}$ (D),

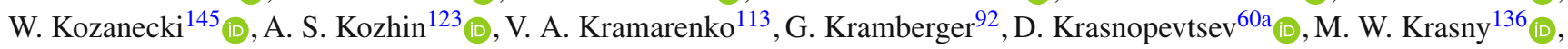
A. $K_{\text {Krasznahorkay }}^{36}$ (D), D. Krauss ${ }^{115}$ (D), J. A. Kremer ${ }^{84 a}$ (D), J. Kretzschmar ${ }^{11}$ (D), P. Krieger ${ }^{167}$ (D), F. Krieter ${ }^{114}$ (D),

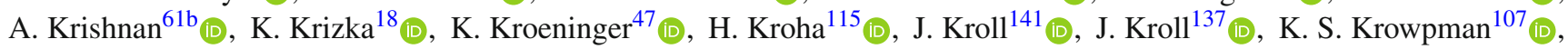

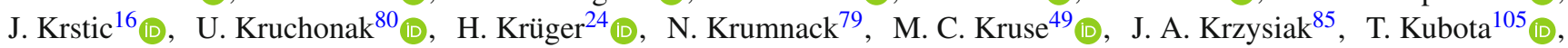

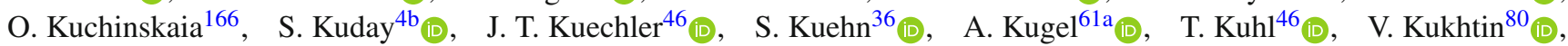

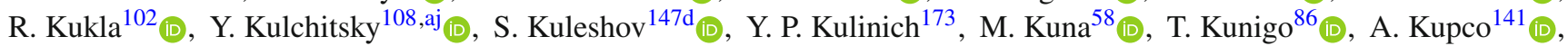
T. Kupfer ${ }^{47}$, O. Kuprash ${ }^{52}$ (D), H. Kurashige ${ }^{83}$ (D), L. L. Kurchaninov ${ }^{168 a}{ }_{(\mathbb{D})}, \quad$ Y. A. Kurochkin ${ }^{108}, \quad$ A. Kurova ${ }^{112}$ (D),

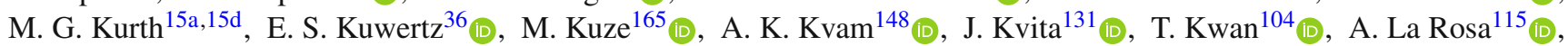

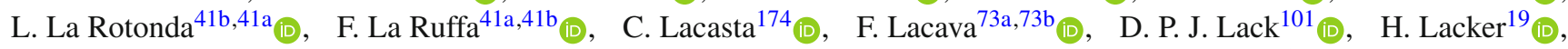

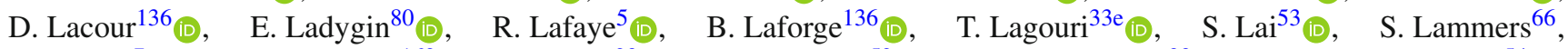

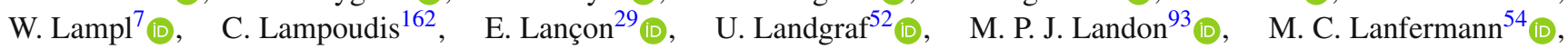

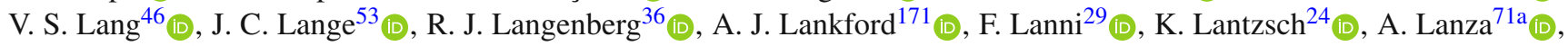

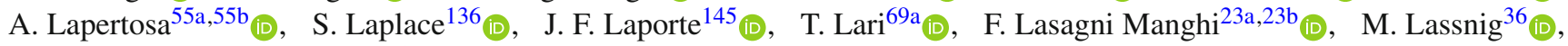

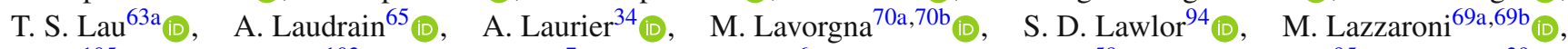
B. Le ${ }^{105}$, E. Le Guirriec ${ }^{102}$ (D), M. LeBlanc ${ }^{7}$ (D), T. LeCompte $^{6}$ (D), F. Ledroit-Guillon ${ }^{58}$ (D), A. C. A. Lee ${ }^{95}$, C. A. Lee ${ }^{29}$ (D),

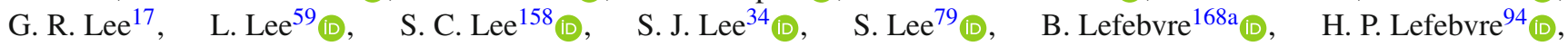
M. Lefebvre ${ }^{176}$ (D), F. Legger ${ }^{114}$ (D), C. Leggett ${ }^{18}$ (D), K. Lehmann ${ }^{152}$ (D), N. Lehmann ${ }^{182}$ (D), G. Lehmann Miotto ${ }^{36}$ (D),

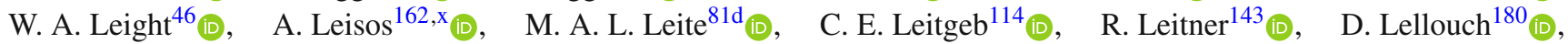

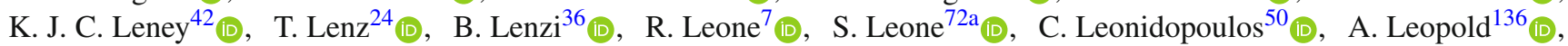

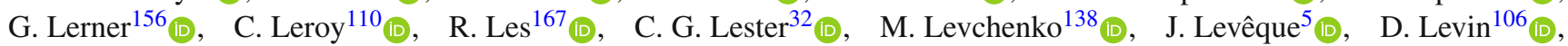

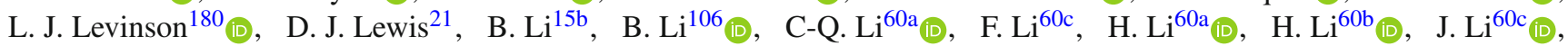




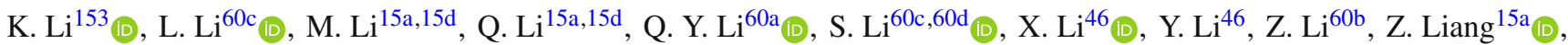

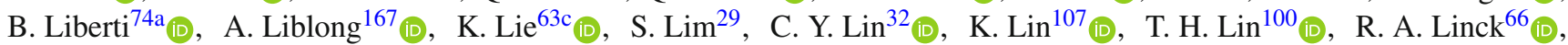

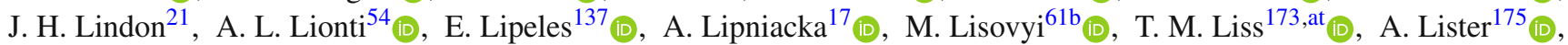

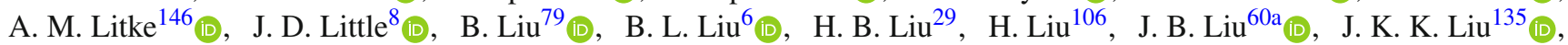

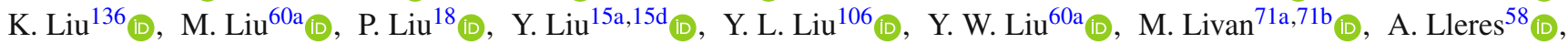

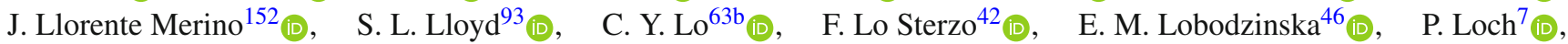

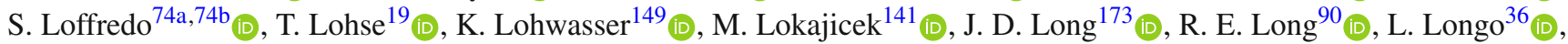
K. A. Looper ${ }^{127}$ (D), J. A. Lopez ${ }^{147 d}$ (D), I. Lopez Paz ${ }^{101}$, A. Lopez Solis ${ }^{149}$ (D) J. Lorenz ${ }^{114}$ (C), N. Lorenzo Martinez ${ }^{5}$ (D),

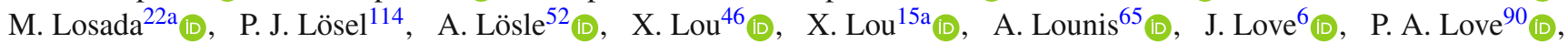

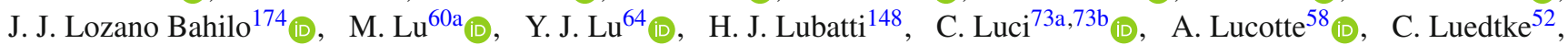
F. Luehring ${ }^{66}$ (D), I. Luise ${ }^{136}$ (D), L. Luminari ${ }^{73 a}$, B. Lund-Jensen ${ }^{154}$ (D), M. S. Lutz ${ }^{103}$ (D), D. Lynn ${ }^{29}$ (D), H. Lyons $^{91}$,

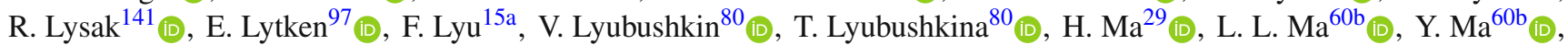

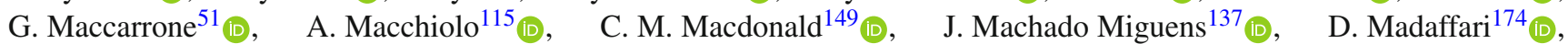

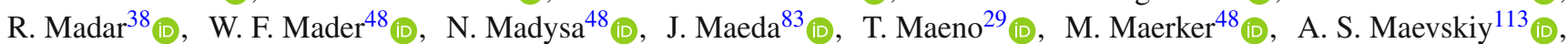

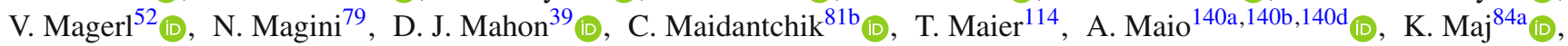
O. Majersky ${ }^{28 a}$ (D) S. Majewski ${ }^{132}$ (D) , Y. Makida ${ }^{82}$, N. Makovec $^{65}$ (D) B. Malaescu ${ }^{136}$ (D) Pa. Malecki ${ }^{85}$ (D) , V. P. Maleev ${ }^{138}$ (D), F. Malek ${ }^{58}$ (D) U. Mallik ${ }^{78}$ (D) D. Malon ${ }^{6}$ (D) , C. Malone ${ }^{32}$, S. Maltezos ${ }^{10}$, S. Malyukov ${ }^{80}$, J. Mamuzic ${ }^{174}$ (D) , G. Mancini ${ }^{51}$ (D), I. Mandić ${ }^{92}$ (D) L. Manhaes de Andrade Filho ${ }^{81 \mathrm{a}}$ (D), I. M. Maniatis ${ }^{162}$ (D), J. Manjarres $\operatorname{Ramos}^{48}$ (D), K. H. Mankinen ${ }^{97}$ (D), A. Mann ${ }^{114}$ (D), A. Manousos ${ }^{77}$ (D), B. Mansoulie ${ }^{145}$ (D), I. Manthos ${ }^{162}$ (D), S. Manzoni ${ }^{120}$ (D), A. Marantis ${ }^{162}$ (D),

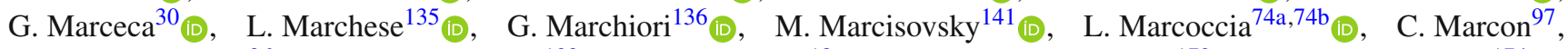

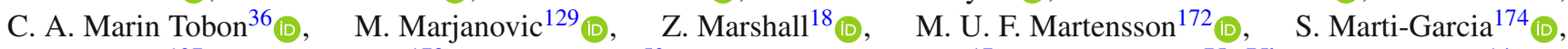
C. B. Martin ${ }^{127}$ (D) T. A. $\operatorname{Martin}^{178}$ (D) V. J. Martin ${ }^{50}$ (D) B. Martin dit $\operatorname{Latour}^{17}$ (D), L. Martinelli ${ }^{75 a, 75 b}$ (D), M. Martinez ${ }^{14, z}$ (D),

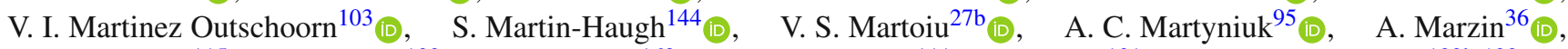

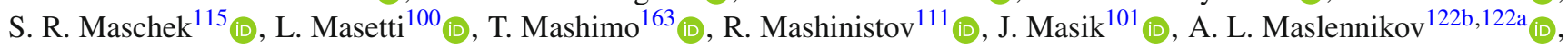
L. Massa ${ }^{74 a, 74 b}$ (D), P. Massarotti ${ }^{70 a, 70 b}$ (D), P. Mastrandrea ${ }^{72 a, 72 b}$ (D), A. Mastroberardino ${ }^{41 a, 41 b}$ (D), T. Masubuchi ${ }^{163}$ (D), D. Matakias ${ }^{10}$, A. Matic ${ }^{14}$ (D) N. Matsuzawa ${ }^{163}$, P. Mättig ${ }^{24}$ (D), J. Maurer ${ }^{27 b}$ (D), B. Maček ${ }^{92}$, D. A. Maximov ${ }^{122 a, 122 b}$ (D), R. Mazini ${ }^{158}$ (D), I. Maznas ${ }^{162}$ (D), S. M. Mazza ${ }^{146}$ (D), S. P. Mc Kee ${ }^{106}$ (D), T. G. McCarthy ${ }^{115}$ (D), W. P. McCormack ${ }^{18}$,

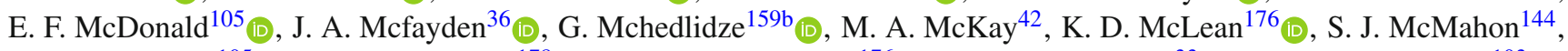

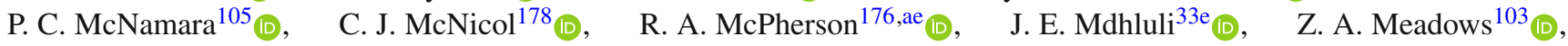
S. Meehan ${ }^{36}$ (D), T. Megy ${ }^{52}$ (D), S. Mehlhase ${ }^{114}$ (D), A. Mehta ${ }^{91}$ (D), T. Meideck ${ }^{58}$ (D) B. Meirose ${ }^{43}$ (D), D. Melini ${ }^{174}$ (D),

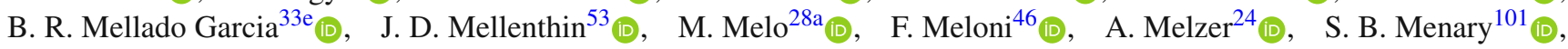

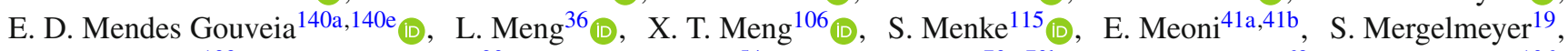

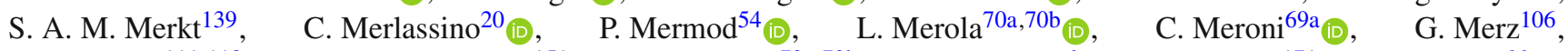

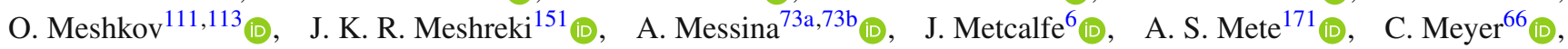

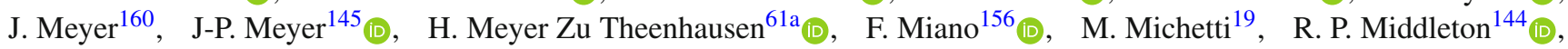

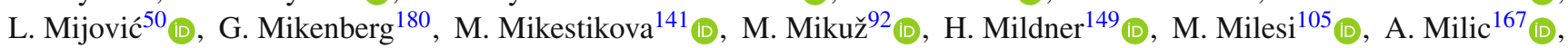
D. A. Millar ${ }^{93}$ (D), D. W. Miller ${ }^{37}$ (D) A. Milov $^{180}$ (D), D. A. Milstead ${ }^{45 a, 45 b}$, R. A. Mina ${ }^{153}$ (D), A. A. Minaenko ${ }^{123}$ (D),

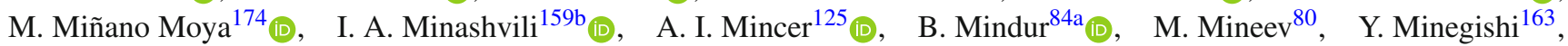
L. M. Mir $^{14}$ (D), A. Mirto ${ }^{68 \mathrm{a}, 68 \mathrm{~b}}$ (D), K. P. Mistry ${ }^{137}$ (D), T. Mitani ${ }^{179}$ (D), J. Mitrevski ${ }^{114}$, V. A. Mitsou ${ }^{174}$ (D), M. Mittal ${ }^{60 \mathrm{c}}$, O. Miu $^{167}$ (D), A. Miucci ${ }^{20}$ (D), P. S. Miyagawa ${ }^{149}$ (D, $\quad$ A. Mizukami ${ }^{82}$ (D), J. U. Mjörnmark ${ }^{97}, \quad$ T. Mkrtchyan ${ }^{184}$ (D) $^{\text {, }}$

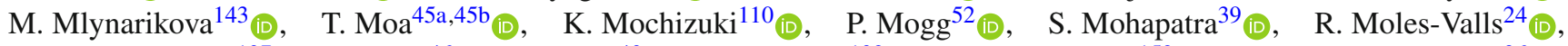
M. C. Mondragon ${ }^{107}$, K. Mönig ${ }^{46}$, J. Monk $^{40}{ }_{(\mathbb{D}}$, E. Monnier ${ }^{102}$ (D) , A. Montalbano ${ }^{152}$ (D), J. Montejo Berlingen ${ }^{36}$ (D),

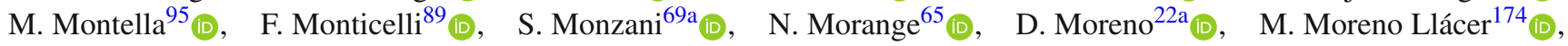
C. Moreno Martinez ${ }^{14}$ (D), P. Morettini ${ }^{55 b}$ (D), M. Morgenstern ${ }^{120}$ (D), S. Morgenstern ${ }^{48}$ (D), D. Mori ${ }^{152}$ (D), M. Morii ${ }^{59}$ (D),

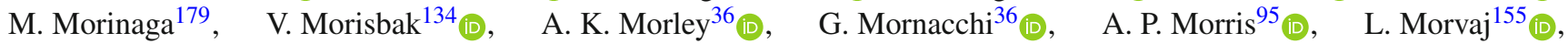

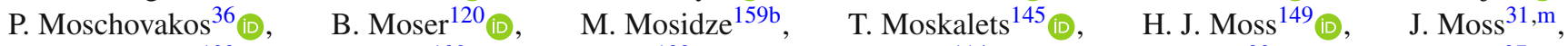
E. J. W. Moyse ${ }^{103}$ (D), S. Muanza ${ }^{102}$ (D), J. Mueller ${ }^{139}$ (D), R. S. P. Mueller ${ }^{114}$, D. Muenstermann ${ }^{90}$ (D), G. A. Mullier ${ }^{97}$ (D), $\begin{array}{lll}\text { D. P. Mungo } & 69 \mathrm{a}, 69 \mathrm{~b} \\ \mathbb{D}\end{array}$

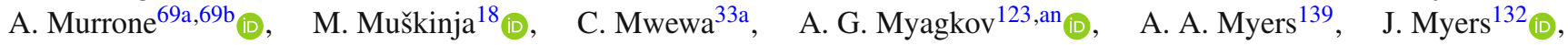

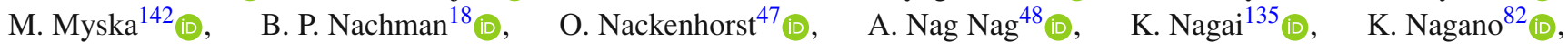

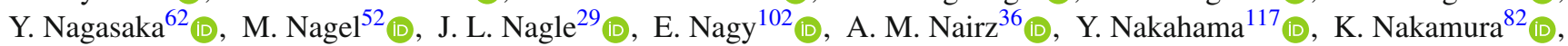

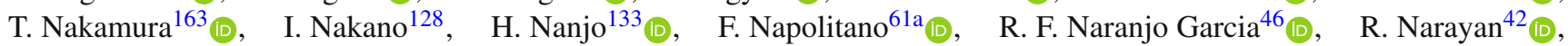

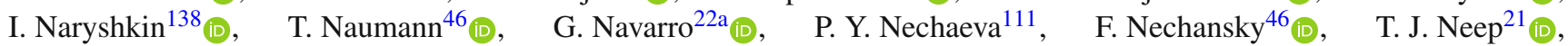




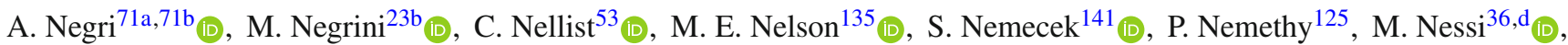
M. S. Neubauer ${ }^{173}$ (D) M. Neumann ${ }^{182}$, R. Newhouse ${ }^{175}$ (I) , P. R. Newman ${ }^{21}$ (D) Y. S. Ng ${ }^{19}$, Y. W. Y. Ng$^{171}$, B. Ngair $^{35 e}$ (D),

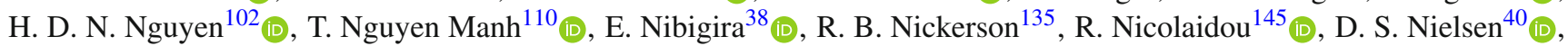

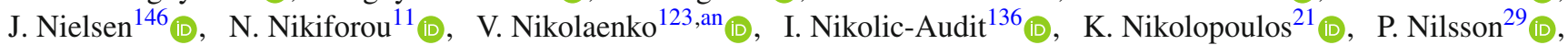

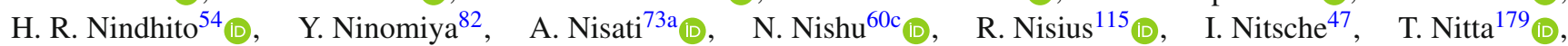
T. Nobe ${ }^{163}$ (1), Y. Noguchi ${ }^{86}$, I. Nomidis ${ }^{136}$ (D), M. A. Nomura ${ }^{29}$, M. Nordberg ${ }^{36}$, N. Norjoharuddeen ${ }^{135}$ (D), T. Novak ${ }^{92}$ (D),

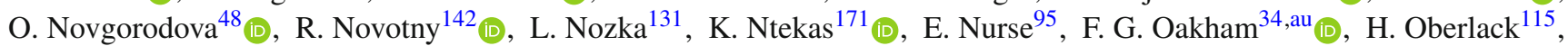

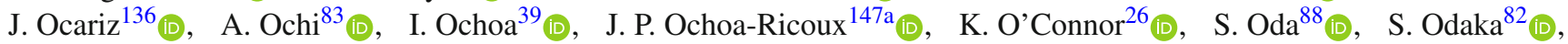

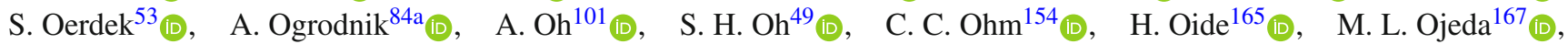
H. Okawa ${ }^{169}$ (D), Y. Okazaki ${ }^{86}$ (D) M. W. O’ Keefe ${ }^{91}$, Y. Okumura ${ }^{163}$ (D), T. Okuyama ${ }^{82}$, A. Olariu ${ }^{27 b}$, L. F. Oleiro Seabra ${ }^{140 a}$, S. A. Olivares Pino ${ }^{147 a}$, D. Oliveira Damazio ${ }^{29}$, J. L. Oliver ${ }^{1}$, M. J. R. Olsson ${ }^{171}$ (D), A. Olszewski ${ }^{85}$ (I) J. Olszowska ${ }^{85}$ (D),

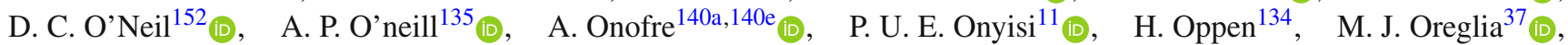

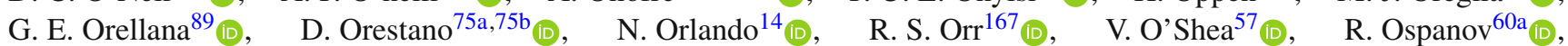

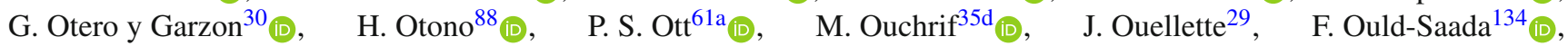

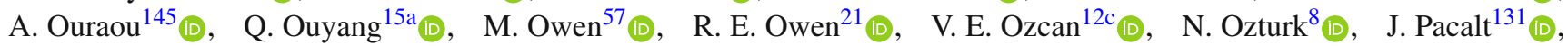
H. A. Pacey ${ }^{32}$ (D) K. Pachal ${ }^{49}$ (D), A. Pacheco Pages ${ }^{14}$ (D), C. Padilla Aranda ${ }^{14}$ (D), S. Pagan Griso ${ }^{18}$ (D), M. Paganini ${ }^{183}$ (D),

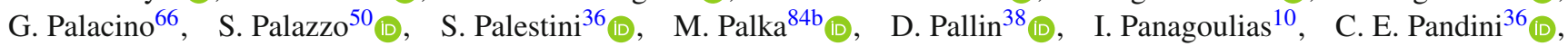

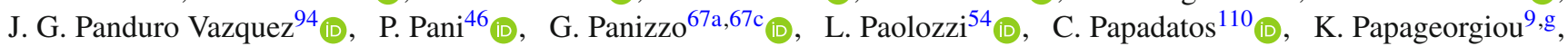

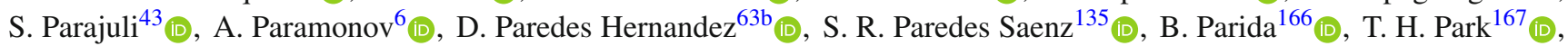

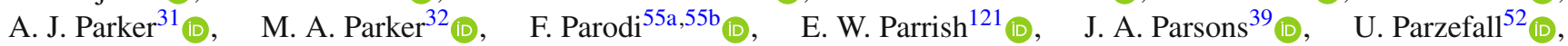

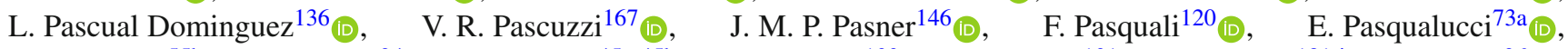

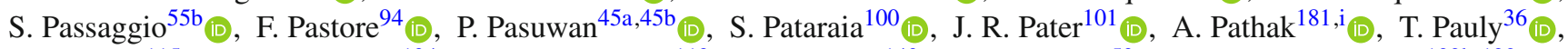

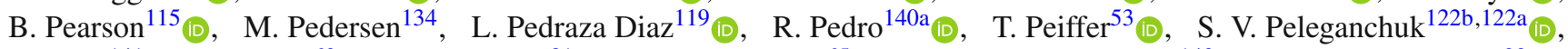

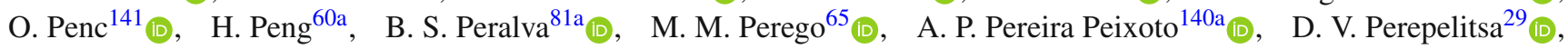

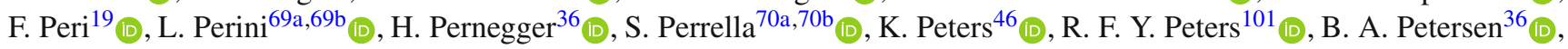

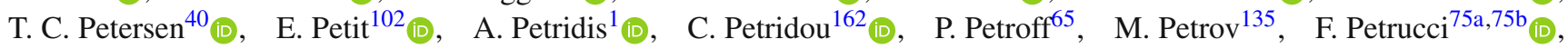

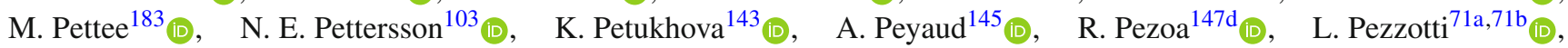
T. Pham ${ }^{105}$ (D), F. H. Phillips ${ }^{107}$ (D), P. W. Phillips ${ }^{144}$ (D), M. W. Phipps ${ }^{173}$ (D), G. Piacquadio ${ }^{155}$ (D), $\quad$ E. Pianori ${ }^{18}$ (D),

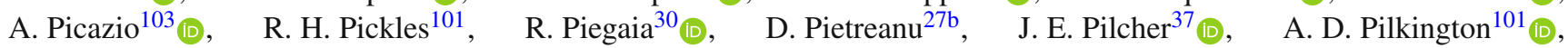

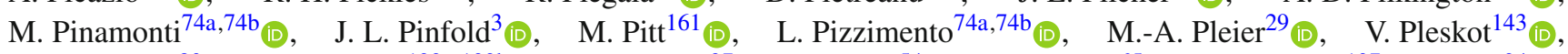
E. Plotnikova ${ }^{80}$, P. Podberezko ${ }^{122 a, 122 b}$ (D), R. Poettgen ${ }^{97}$ (D), R. Poggi ${ }^{54}$ (D) L. Poggioli ${ }^{65}$, I. Pogrebnyak ${ }^{107}$, D. Pohl ${ }^{24}$ (D),

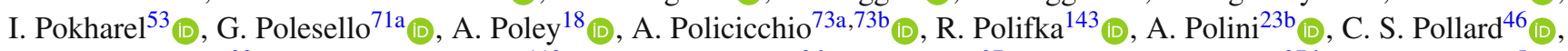

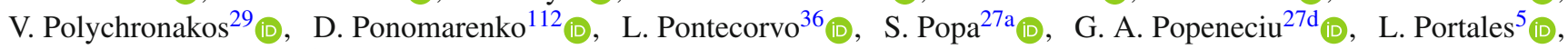

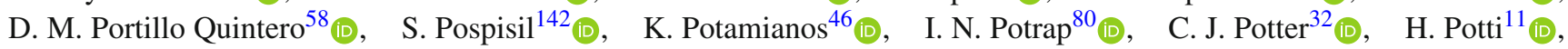

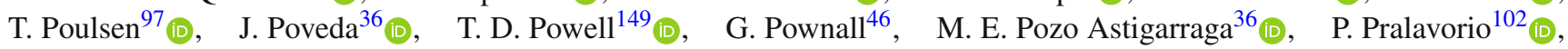

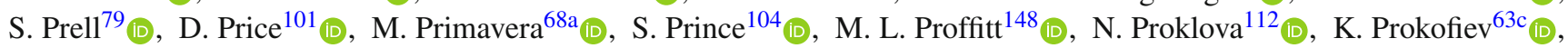
F. Prokoshin ${ }^{80}$ (D), S. Protopopescu ${ }^{29}, \quad$ J. Proudfoot $^{6}$ (D) $\quad$ M. Przybycien ${ }^{84 a}$ (D), D. Pudzha ${ }^{138}$ (D), A. Puri ${ }^{173}$ (D),

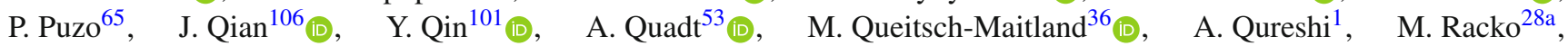
P. Rados $^{105}$, F. Ragusa ${ }^{69 a, 69 b}{ }_{(\mathbb{D})}, \quad$ G. Rahal ${ }^{98}$ (D), J. A. Raine ${ }^{54}$ (D), S. Rajagopalan ${ }^{29}$ (D), A. Ramirez Morales ${ }^{93}$,

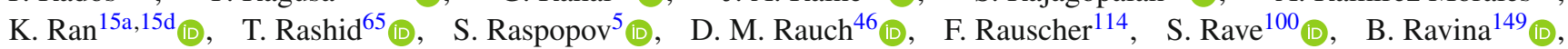

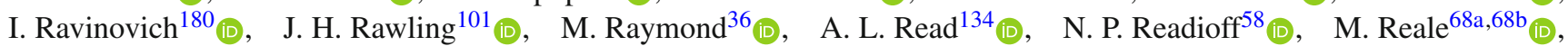
D. M. Rebuzzi ${ }^{71 a, 71 b}$ (D), A. Redelbach ${ }^{177}$ (D), G. Redlinger ${ }^{29}$ (D), K. Reeves ${ }^{43}$ (D), L. Rehnisch ${ }^{19}$ (D), J. Reichert ${ }^{137}$ (D),

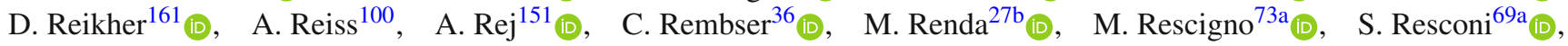
E. D. Resseguie ${ }^{137}$ (D), S. Rettie ${ }^{175}$ (D), B. Reynolds ${ }^{127}$, E. Reynolds ${ }^{21}$ (D), O. L. Rezanova ${ }^{122 a, 122 b}$ (D), P. Reznicek ${ }^{143}$ (D),

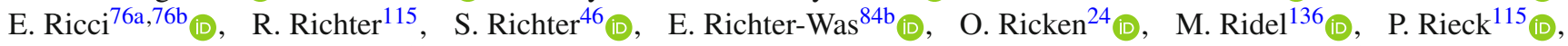

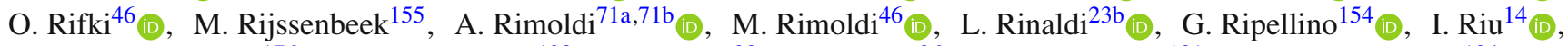

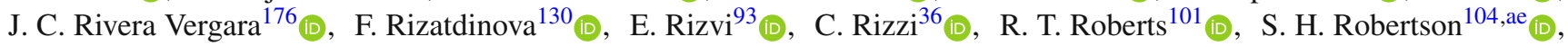

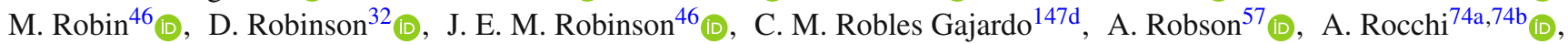

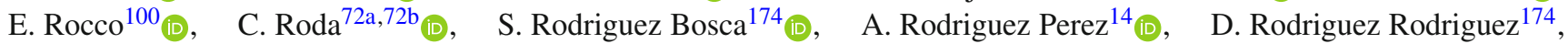

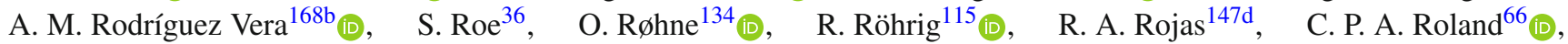
J. Roloff ${ }^{29}$ (D) A. Romaniouk ${ }^{112}$ (D), M. Romano ${ }^{23 a, 23 b}$ (D) N. Rompotis ${ }^{91}$ (D), M. Ronzani ${ }^{125}$, L. Roos ${ }^{136}$ (D), S. Rosati ${ }^{73 a}$ (D),

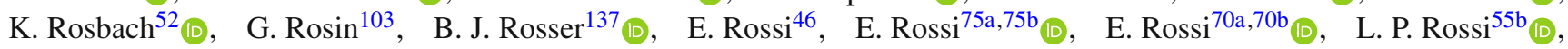

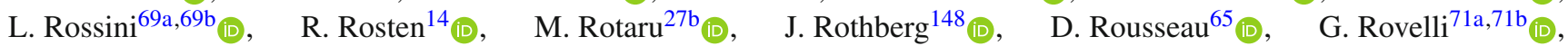




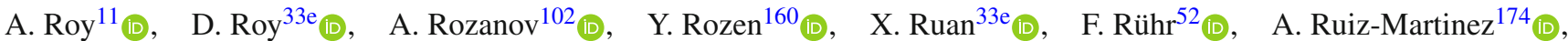

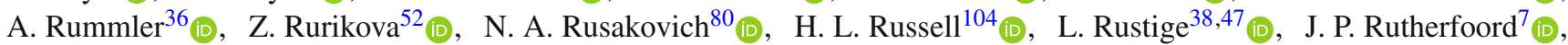

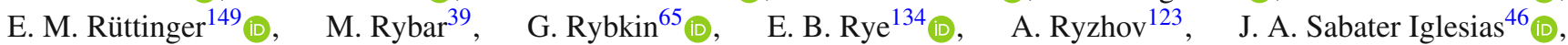

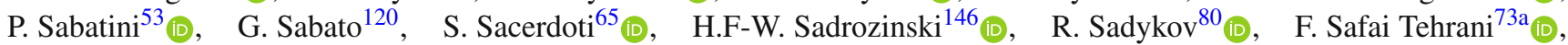
B. Safarzadeh Samani ${ }^{156}$ (D) , P. Saha ${ }^{121}$ (D), S. Saha ${ }^{104}$ (D), M. Sahinsoy ${ }^{61 a}$ (D), A. Sahu ${ }^{182}$ (D), M. Saimpert ${ }^{46}$ (D), M. Saito ${ }^{163}$ (D), T. Saito ${ }^{163}$ (D) H. Hakamoto ${ }^{163}$ (D), A. Sakharov ${ }^{125, a_{\text {(D) }} \text {, D. Salamani }}{ }^{54}$, G. Salamanna ${ }^{15 a, 75 b}$ (D), J. E. Salazar Loyola ${ }^{147 d}$,

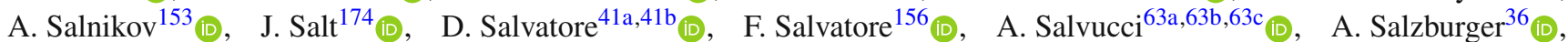
J. Samarati ${ }^{36}$, D. Sammel ${ }^{52}$ (D), D. Sampsonidis ${ }^{162}$, D. Sampsonidou ${ }^{162}$ (D) J. Sánchez ${ }^{174}{ }_{(D)}$, A. Sanchez Pineda ${ }^{36,67 a, 67 c}{ }_{\mathbb{D}}$,

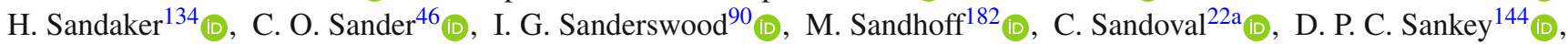

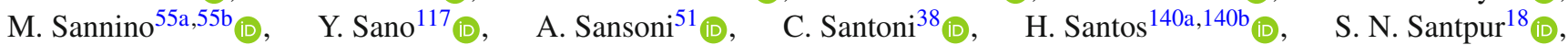

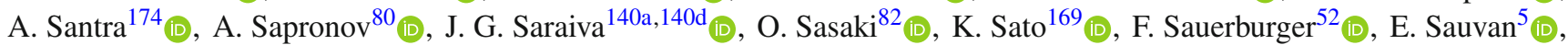

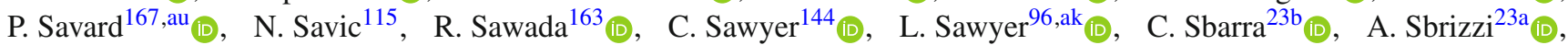
T. Scanlon ${ }^{95}$ (D), J. Schaarschmidt ${ }^{148}$ (D), P. Schacht ${ }^{115}$ (D), B. M. Schachtner ${ }^{114}$ (D), D. Schaefer ${ }^{37}$ (D),$\quad$ L. Schaefer ${ }^{137}$ (D),

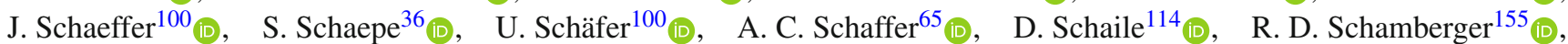
E. Schanet ${ }^{114}$, N. Scharmberg ${ }^{101}$ (D), V. A. Schegelsky ${ }^{138}$ (D), D. Scheirich ${ }^{143}$ (D), F. Schenck ${ }^{19}$ (D), M. Schernau ${ }^{171}$ (D),

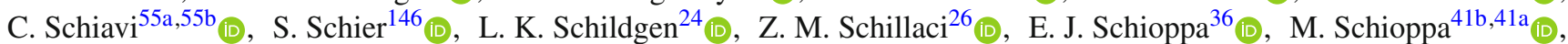

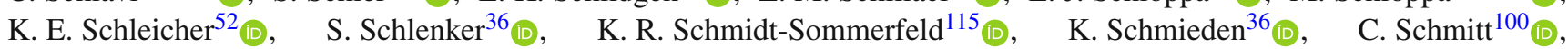

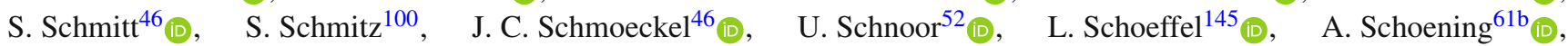
P. G. Scholer ${ }^{52}$ (i), E. Schopf ${ }^{135}$ (i), M. Schott ${ }^{100}$ (i), J. F. P. Schouwenberg ${ }^{119}$ (i) , J. Schovancova ${ }^{36}$ (i), S. Schramm ${ }^{54}$ (i), F. Schroeder ${ }^{182}$ (1) A. Schulte ${ }^{100}$ (1) H-C. Schultz-Coulon ${ }^{61 \mathrm{a}}$ (D), M. Schumacher ${ }^{52}$ (I) B. A. Schumm ${ }^{146}$ (D), Ph. Schune ${ }^{145}$,

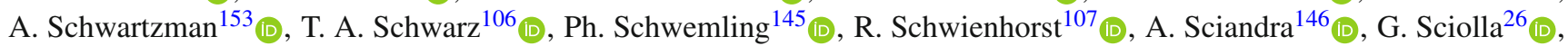

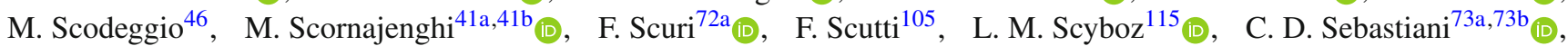

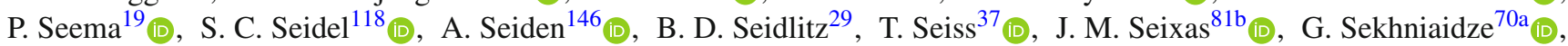

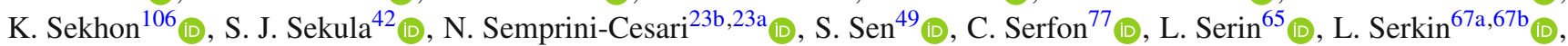

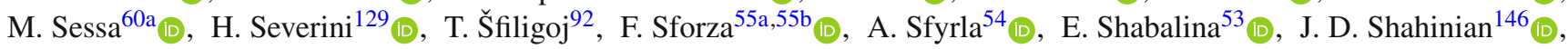

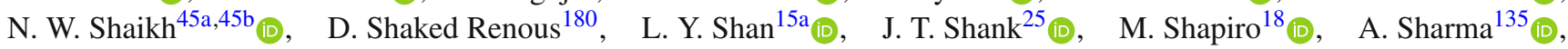

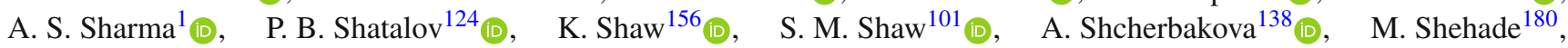
Y. Shen ${ }^{129}$, A. D. Sherman ${ }^{25}$, P. Sherwood ${ }^{95}$ (D) L. Shi ${ }^{158}$,as $\mathbb{C}$, S. Shimizu ${ }^{82}$ (D) C. O. Shimmin ${ }^{183}$ (D), Y. Shimogama ${ }^{179}$,

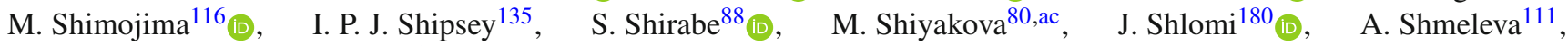

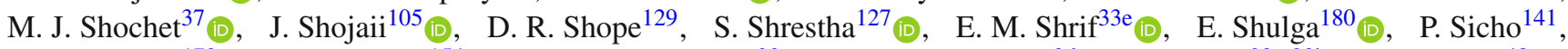

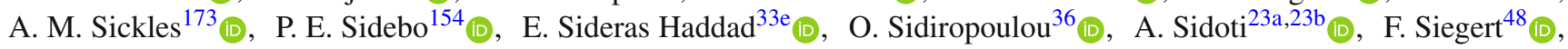

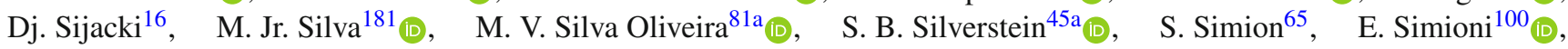

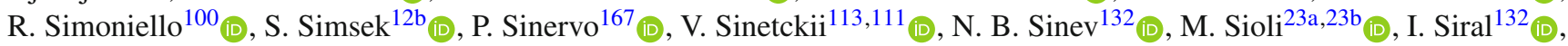

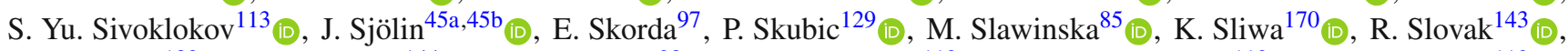

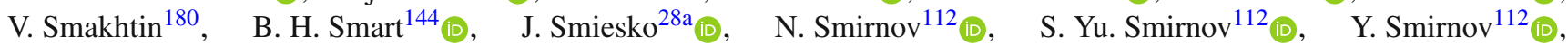

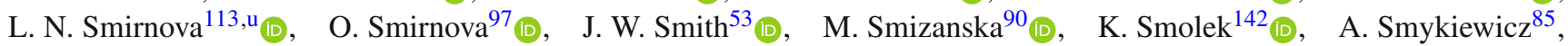

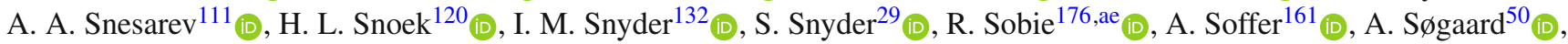
F. Sohns ${ }^{53}$ (D) C. A. Solans Sanchez ${ }^{36}$ (D) E. Yu. Soldatov ${ }^{112}$ (D), U. Soldevila ${ }^{174}$ (D), A. A. Solodkov ${ }^{123}$ (D), A. Soloshenko ${ }^{80}$ (D), O. V. Solovyanov ${ }^{123}$ (D), V. Solovyev ${ }^{138}$ (D) $\quad$ P. Sommer $^{149}$ (D), H. Son ${ }^{170}$ (D), W. Song ${ }^{144}$ (D), W. Y. Song ${ }^{168 b}$ (D),

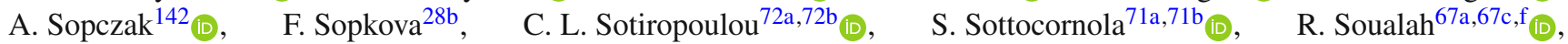

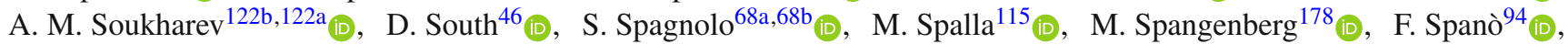

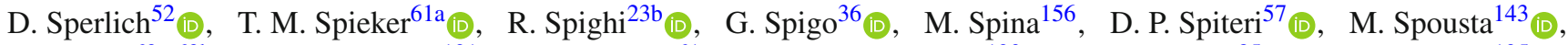

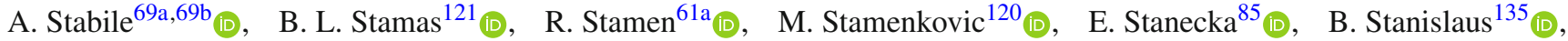

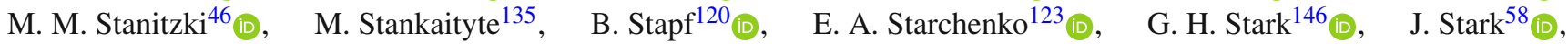

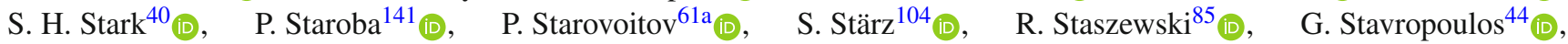

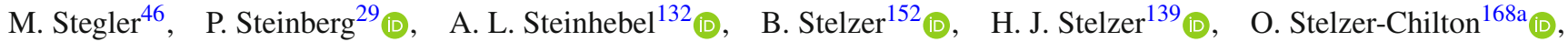

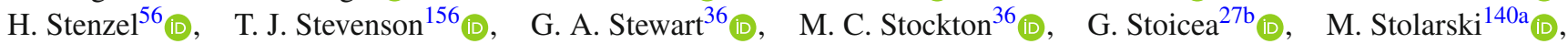

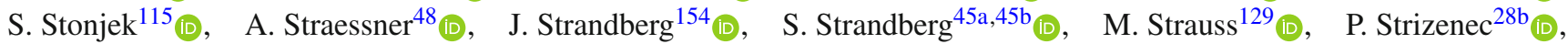

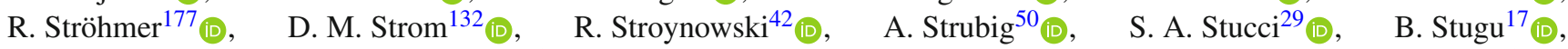

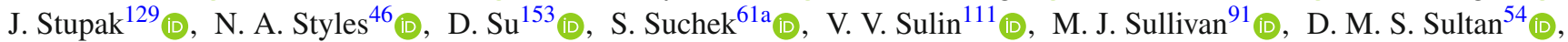

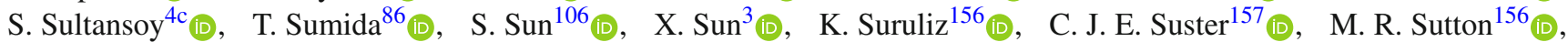

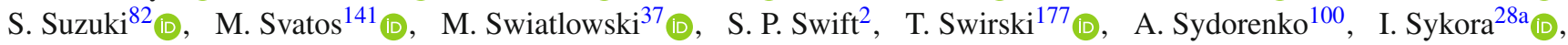

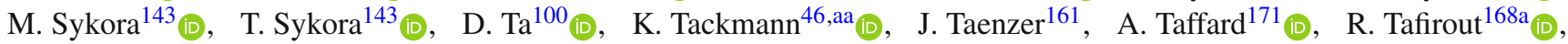


H. Takai ${ }^{29}$ (D), R. Takashima ${ }^{87}, \quad$ K. Takeda ${ }^{83}$ (D), T. Takeshita ${ }^{150}$, E. P. Takeva ${ }^{50}$ (D), Y. Takubo ${ }^{82}$ (D), M. Talby ${ }^{102}$ (D), A. A. Talyshev122b,122a, N. M. Tamir ${ }^{161}$, J. Tanaka ${ }^{163}$ (D), M. Tanaka ${ }^{165}, \quad$ R. Tanaka ${ }^{65}$ (D), S. Tapia Araya ${ }^{173}$ (D),

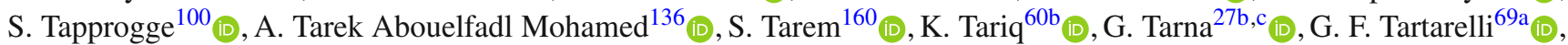
P. Tas ${ }^{143}$ (D), M. Tasevsky ${ }^{141}$ (D), T. Tashiro ${ }^{86}$, E. Tassi ${ }^{41 b, 41 a}$ (D), A. Tavares Delgado ${ }^{140 a, 140 b}$, Y. Tayalati ${ }^{35 e}$, A. J. Taylor ${ }^{50}$ (D), G. N. Taylor ${ }^{105}$ (D), W. Taylor ${ }^{168 b}$ (D), A. S. Te ${ }^{90}, \quad$ R. Teixeira De Lima ${ }^{153}$ (D), P. Teixeira-Dias ${ }^{94}$ (D), H. Ten Kate ${ }^{36}$,

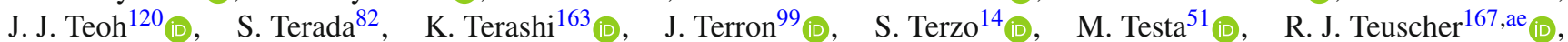
S. J. Thais ${ }^{183}$ (D), T. Theveneaux-Pelzer ${ }^{46}$ (D), F. Thiele ${ }^{40}$ (D), D. W. Thomas ${ }^{94}$, J. O. Thomas ${ }^{42}$, J. P. Thomas ${ }^{21}$ (D), A. S. Thompson ${ }^{57}$, P. D. Thompson ${ }^{21}$ (D) L. A. Thomsen ${ }^{183}$ (D), E. Thomson ${ }^{137}$ (I) , E. J. Thorpe ${ }^{93}$ (D), R. E. Ticse Torres ${ }^{53}$ (D), V. O. Tikhomirov ${ }^{111, a 0}\left(\mathbb{D}, \quad\right.$ Yu. A. Tikhonov ${ }^{122 a, 122 b}{ }_{(\mathbb{D})}, \quad$ S. Timoshenko ${ }^{112}, \quad$ P. Tipton $^{183}$ (D), S. Tisserant ${ }^{102}$ (D),

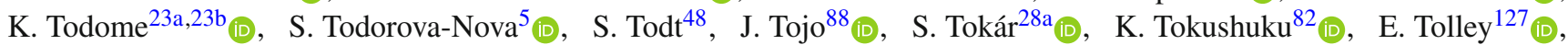

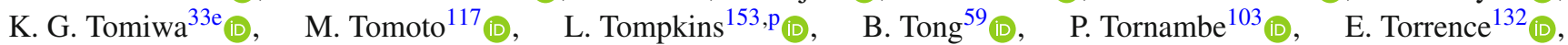

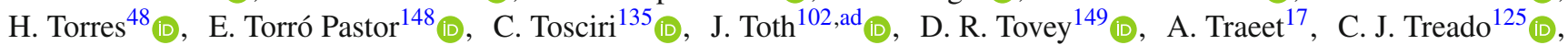

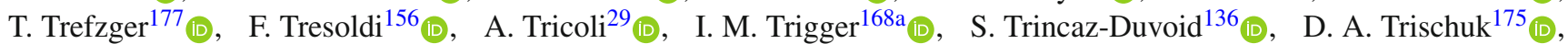

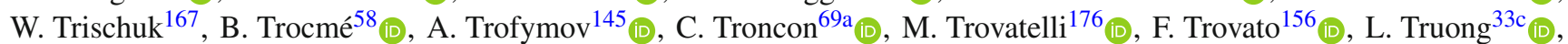
M. Trzebinski ${ }^{85}$ (D), A. Trzupek $^{85}$ (D),$\quad$ F. Tsai ${ }^{46}$ (D), J.C-L. Tseng ${ }^{135}$ (D), P. V. Tsiareshka ${ }^{108, a j}, \quad$ A. Tsirigotis ${ }^{162, x_{(\mathbb{D}},}$ V. Tsiskaridze $\mathrm{e}^{155}$ (D), E. G. Tskhadadze ${ }^{159 \mathrm{a}}, \quad$ M. Tsopoulou ${ }^{162}$, I. I. Tsukerman ${ }^{124}$ (D), V. Tsulaia ${ }^{18}$ (D), S. Tsuno ${ }^{82}$ (D),

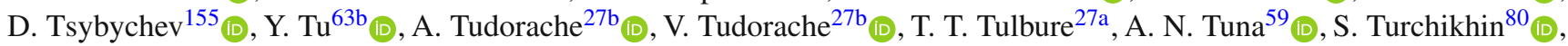
D. Turgeman ${ }^{180}$, I. Turk Cakir ${ }^{4 b, v}$, R. J. Turner ${ }^{21}$, R. T. Turra ${ }^{69 a}$ (D), P. M. Tuts ${ }^{39}$ (D), S. Tzamarias ${ }^{162}$, E. Tzovara ${ }^{100}{ }^{10}$,

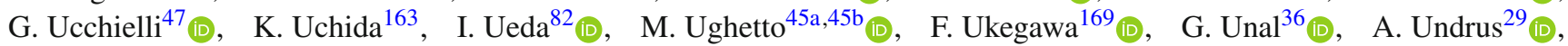

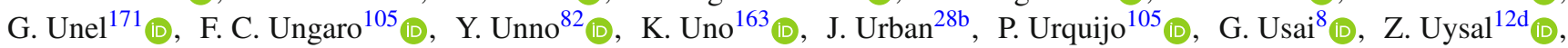
V. Vacek ${ }^{142}$, B. Vachon ${ }^{104}$ (D), K. O. H. Vadla ${ }^{134}$ (D), A. Vaidya ${ }^{95}$ (D), C. Valderanis ${ }^{114}$ (D), E. Valdes Santurio ${ }^{45 a, 45 b}$ (D), $_{\text {, }}$,

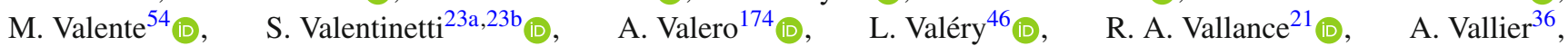

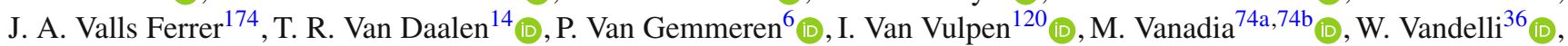

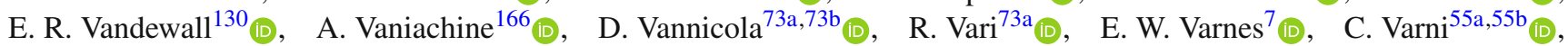

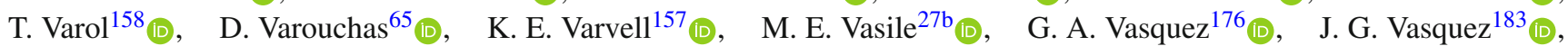
F. Vazeille ${ }^{38}$ (D), D. Vazquez Furelos ${ }^{14}$, T. Vazquez Schroeder $^{36}$ (D) J. Veatch ${ }^{53}$ (D), V. Vecchio ${ }^{75 a, 75 b}$ (D), M. J. Veen ${ }^{120}{ }_{(\mathbb{D})}$,

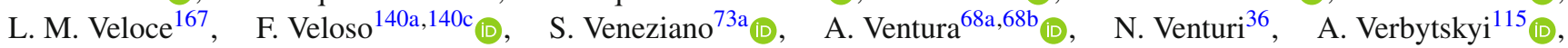

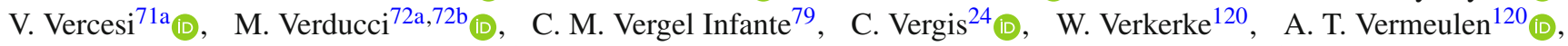

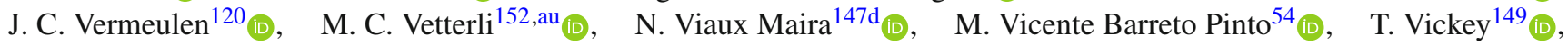

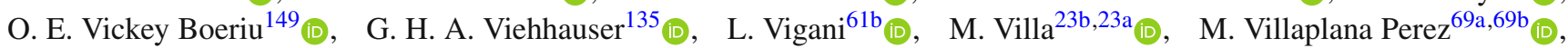

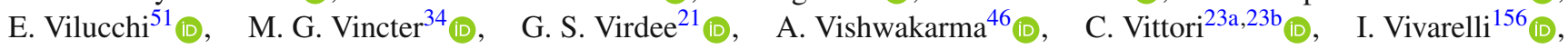
M. Vogel ${ }^{182}$, P. Vokac ${ }^{142}$ (D), S. E. von Buddenbrock ${ }^{3 \mathrm{e}^{\circ}}$,, E. Von Toerne ${ }^{24}$ (D), V. Vorobel ${ }^{143}$ (D), K. Vorobev ${ }^{112}$ (D), M. $\operatorname{Vos}^{174}$ (D) J. H. Vossebeld ${ }^{91}$ (D), M. Vozak ${ }^{101}$, N. Vranjes ${ }^{16}$ (D) M. Vranjes Milosavljevic ${ }^{16}$ (D), V. Vrba ${ }^{142}$, M. Vreeswijk $^{120}$, R. Vuillermet ${ }^{36}$ (1) I. Vukotic ${ }^{37}$ (D), P. Wagner ${ }^{24}$ (D), W. Wagner ${ }^{182}$ (D), J. Wagner-Kuhr ${ }^{114}$ (D), S. Wahdan ${ }^{182}$ (1) , H. Wahlberg ${ }^{89}$ (1), V. M. Walbrecht ${ }^{11^{15}}$, J. Walder ${ }^{90}$ (i), R. Walker ${ }^{114}$ (I), S. D. Walker ${ }^{94}$, W. Walkowiak ${ }^{151}$ (i), V. Wallangen ${ }^{45 a}, 45 \mathrm{~b}$,

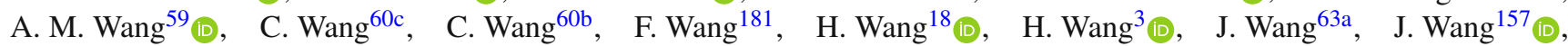

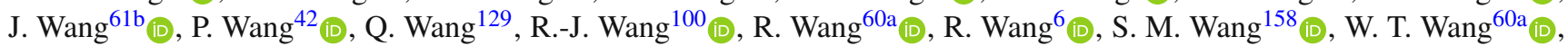

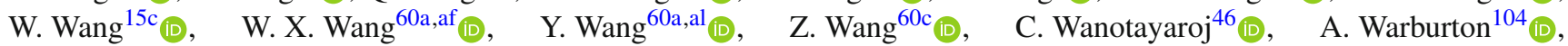
C. P. Ward ${ }^{32}$ (D), D. R. Wardrope ${ }^{95}$ (D) N. Warrack ${ }^{57}$ (D), A. Washbrook ${ }^{50}$, A. T. Watson ${ }^{21}$ (D) M. F. Watson ${ }^{21}$ (D) , G. Watts ${ }^{148}$ (D),

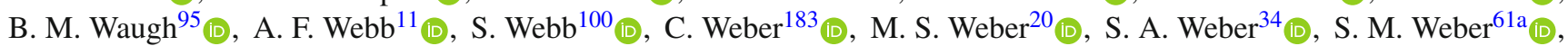
A. R. Weidberg ${ }^{135}$ (D) J. Weingarten ${ }^{47}$ (D), M. Weirich ${ }^{100}$ (D), C. Weiser ${ }^{52}$ (D), P. S. Wells ${ }^{36}$ (D), T. Wenaus ${ }^{29}$ (D) T. Wengler ${ }^{36}$ (D),

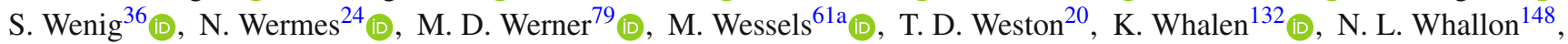

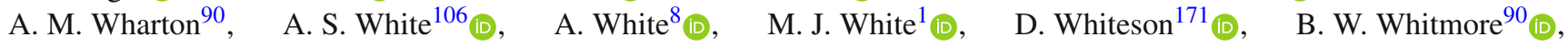
W. Wiedenmann ${ }^{181}$ (D), M. Wielers ${ }^{144}$ (D), N. Wieseotte ${ }^{100}$, C. Wiglesworth ${ }^{40}$ (D) L. A. M. Wiik-Fuchs ${ }^{52}$ (D), F. Wilk ${ }^{101}$, H. G. Wilkens ${ }^{36}$ (D) L. J. Wilkins ${ }^{94}$ (1) H. H. Williams ${ }^{137}$, S. Williams ${ }^{32}$, C. Willis ${ }^{107}$, S. Willocq ${ }^{103}$ (1), J. A. Wilson ${ }^{21}$, I. Wingerter-Seez ${ }^{5}$ (D), E. Winkels ${ }^{156}$ (D), F. Winklmeier ${ }^{132}$ (D), O. J. Winston ${ }^{156}$ (D), B. T. Winter ${ }^{52}$ (D), M. Wittgen ${ }^{153}$, M. Wobisch ${ }^{96}$ (D) A. Wolf ${ }^{100}$ (D), T. M. H. Wolf ${ }^{120}$ (D), R. Wolff ${ }^{102}$ (D), R. Wölker ${ }^{135}$ (), J. Wollrath ${ }^{52}$, M. W. Wolter ${ }^{85}$ (D),

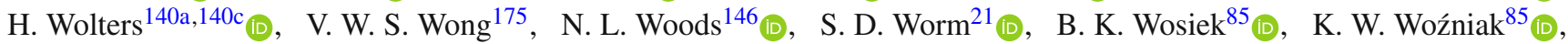

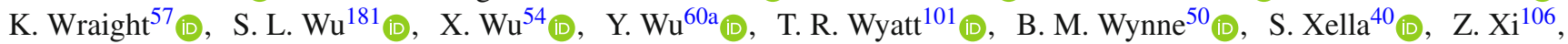

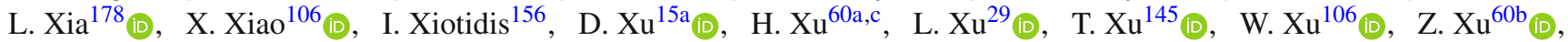

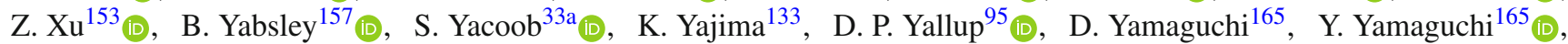
A. Yamamoto ${ }^{82}$ (D) M. Yamatani ${ }^{163}$, T. Yamazaki ${ }^{163}$ (D), Y. Yamazaki ${ }^{83}$ (D), Z. Yan ${ }^{25}$ (D) , H. J. Yang ${ }^{60 c, 60 d}$ (I) , H. T. Yang ${ }^{18}$ (D),

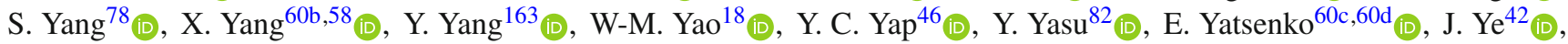


S. Ye $^{29}$ (D) I. Yeletskikh ${ }^{80}$ (D), M. R. Yexley ${ }^{90}$ (D) E. Yigitbasi ${ }^{25}$ (D), K. Yorita ${ }^{179}$ (D), K. Yoshihara $^{137}$ (D), C. J. S. Young ${ }^{36}$ (D),

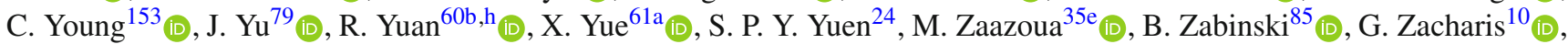
E. Zaffaroni ${ }^{54}\left(\mathbb{D}\right.$, J. Zahreddine ${ }^{136}{ }_{(\mathbb{D})}$, A. M. Zaitsev ${ }^{123, \text { an }}$ (D), T. Zakareishvili ${ }^{159 b}$ (D), N. Zakharchuk ${ }^{34}$ (D), S. Zambito ${ }^{59}$ (D),

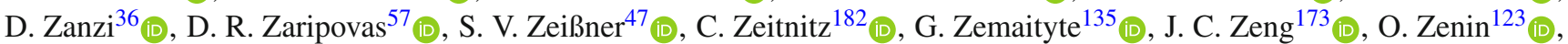

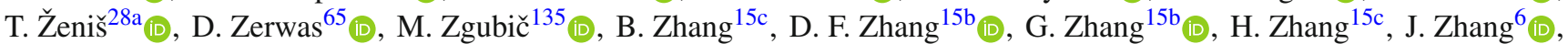

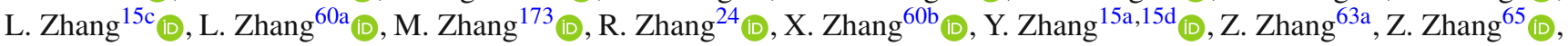
P. Zhao ${ }^{49}$ (1), Y. Zhao ${ }^{60 b}$, Z. Zhao ${ }^{60 a}$, A. Zhemchugov ${ }^{80}$ (D), Z. Zheng ${ }^{106}$, D. Zhong ${ }^{173}$ (D), B. Zhou ${ }^{106}$, C. Zhou $^{181}{ }^{(1)}$,

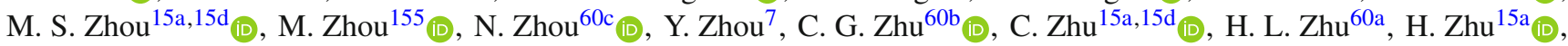

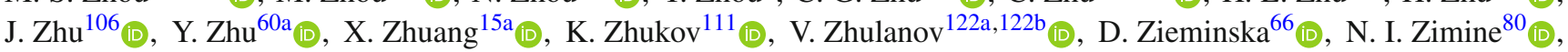

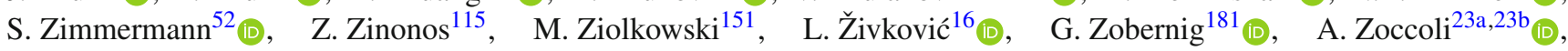
K. Zoch ${ }^{53}$ (D) T. G. Zorbas ${ }^{149}$ (D), R. Zou ${ }^{37}$ (D) L. Zwalinski ${ }^{36}$

${ }^{1}$ Department of Physics, University of Adelaide, Adelaide, Australia

${ }^{2}$ Physics Department, SUNY Albany, Albany, NY, USA

${ }^{3}$ Department of Physics, University of Alberta, Edmonton, AB, Canada

4 (a) Department of Physics, Ankara University, Ankara, Turkey; ${ }^{(b)}$ Istanbul Aydin University, Istanbul, Turkey; ${ }^{(c)}$ Division of Physics, TOBB University of Economics and Technology, Ankara, Turkey

${ }^{5}$ LAPP, Université Grenoble Alpes, Université Savoie Mont Blanc, CNRS/IN2P3, Annecy, France

${ }^{6}$ High Energy Physics Division, Argonne National Laboratory, Argonne, IL, USA

${ }^{7}$ Department of Physics, University of Arizona, Tucson, AZ, USA

${ }^{8}$ Department of Physics, University of Texas at Arlington, Arlington, TX, USA

${ }^{9}$ Physics Department, National and Kapodistrian University of Athens, Athens, Greece

${ }^{10}$ Physics Department, National Technical University of Athens, Zografou, Greece

${ }^{11}$ Department of Physics, University of Texas at Austin, Austin, TX, USA

12 (a) Bahcesehir University, Faculty of Engineering and Natural Sciences, Istanbul, Turkey; ${ }^{(b)}$ Istanbul Bilgi University,

Faculty of Engineering and Natural Sciences, Istanbul, Turkey; ${ }^{\left({ }^{c}\right)}$ Department of Physics, Bogazici University, Istanbul,

Turkey; (d) Department of Physics Engineering, Gaziantep University, Gaziantep, Turkey

${ }^{13}$ Institute of Physics, Azerbaijan Academy of Sciences, Baku, Azerbaijan

${ }^{14}$ Institut de Física d'Altes Energies (IFAE), Barcelona Institute of Science and Technology, Barcelona, Spain

15 (a) Institute of High Energy Physics, Chinese Academy of Sciences, Beijing, China; ${ }^{(b)}$ Physics Department, Tsinghua

University, Beijing, China; ${ }^{(c)}$ Department of Physics, Nanjing University, Nanjing, China; ${ }^{(d)}$ University of Chinese

Academy of Science (UCAS), Beijing, China

${ }^{16}$ Institute of Physics, University of Belgrade, Belgrade, Serbia

${ }^{17}$ Department for Physics and Technology, University of Bergen, Bergen, Norway

${ }^{18}$ Physics Division, Lawrence Berkeley National Laboratory and University of California, Berkeley, CA, USA

${ }^{19}$ Institut für Physik, Humboldt Universität zu Berlin, Berlin, Germany

${ }^{20}$ Albert Einstein Center for Fundamental Physics and Laboratory for High Energy Physics, University of Bern, Bern, Switzerland

${ }^{21}$ School of Physics and Astronomy, University of Birmingham, Birmingham, UK

22 (a) Facultad de Ciencias y Centro de Investigaciónes, Universidad Antonio Nariño, Bogotá, Colombia; ${ }^{(b)}$ Departamento de Física, Universidad Nacional de Colombia, Bogotá, Colombia, Colombia

23 (a) Dipartimento di Fisica, INFN Bologna and Universita' di Bologna, Bologna, Italy; ${ }^{(b)}$ INFN Sezione di Bologna, Bologna, Italy

${ }^{24}$ Physikalisches Institut, Universität Bonn, Bonn, Germany

${ }^{25}$ Department of Physics, Boston University, Boston, MA, USA

${ }^{26}$ Department of Physics, Brandeis University, Waltham, MA, USA

27 (a) Transilvania University of Brasov, Brasov, Romania; ${ }^{(b)}$ Horia Hulubei National Institute of Physics and Nuclear

Engineering, Bucharest, Romania; ${ }^{(c)}$ Department of Physics, Alexandru Ioan Cuza University of Iasi, Iasi, Romania

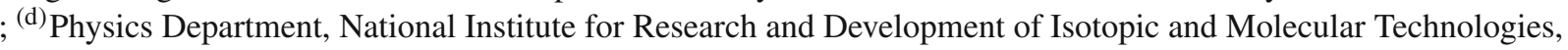

Cluj-Napoca, Romania; ${ }^{(e)}$ University Politehnica Bucharest, Bucharest, Romania; ${ }^{(f)}$ West University in Timisoara,

Timisoara, Romania

28 (a) Faculty of Mathematics, Physics and Informatics, Comenius University, Bratislava, Slovakia; ${ }^{(b)}$ Department of

Subnuclear Physics, Institute of Experimental Physics of the Slovak Academy of Sciences, Kosice, Slovak Republic 
${ }^{29}$ Physics Department, Brookhaven National Laboratory, Upton, NY, USA

${ }^{30}$ Departamento de Física, Universidad de Buenos Aires, Buenos Aires, Argentina

${ }^{31}$ California State University, CA, USA

${ }^{32}$ Cavendish Laboratory, University of Cambridge, Cambridge, UK

33 (a) Department of Physics, University of Cape Town, Cape Town, South Africa; ${ }^{(b)}$ iThemba Labs, Western Cape, South Africa; ${ }^{(c)}$ Department of Mechanical Engineering Science, University of Johannesburg, Johannesburg, South Africa

; ${ }^{(d)}$ Department of Physics, University of South Africa, Pretoria, South Africa; ${ }^{(e)}$ School of Physics, University of the Witwatersrand, Johannesburg, South Africa

${ }^{34}$ Department of Physics, Carleton University, Ottawa, ON, Canada

35 (a) Faculté des Sciences Ain Chock, Réseau Universitaire de Physique des Hautes Energies - Université Hassan II, Casablanca, Morocco; ${ }^{(b)}$ Faculté des Sciences, Université Ibn-Tofail, Kénitra, Morocco; ${ }^{(c)}$ Faculté des Sciences Semlalia, Université Cadi Ayyad, LPHEA-Marrakech, Marrakesh, Morocco; (d) Faculté des Sciences, Université Mohamed Premier and LPTPM, Oujda, Morocco; ${ }^{(e)}$ Faculté des sciences, Université Mohammed V, Rabat, Morocco

${ }^{36}$ CERN, Geneva, Switzerland

${ }^{37}$ Enrico Fermi Institute, University of Chicago, Chicago, IL, USA

${ }^{38}$ LPC, Université Clermont Auvergne, CNRS/IN2P3, Clermont-Ferrand, France

${ }^{39}$ Nevis Laboratory, Columbia University, Irvington, NY, USA

${ }^{40}$ Niels Bohr Institute, University of Copenhagen, Copenhagen, Denmark

41 (a) Dipartimento di Fisica, Università della Calabria, Rende, Italy; ${ }^{(b)}$ INFN Gruppo Collegato di Cosenza, Laboratori Nazionali di Frascati, Italy

${ }^{42}$ Physics Department, Southern Methodist University, Dallas, TX, USA

${ }^{43}$ Physics Department, University of Texas at Dallas, Richardson, TX, USA

${ }^{44}$ National Centre for Scientific Research "Demokritos", Agia Paraskevi, Greece

45 (a) Department of Physics, Stockholm University, Stockholm, Sweden; ${ }^{(b)}$ Oskar Klein Centre, Stockholm, Sweden

${ }^{46}$ Deutsches Elektronen-Synchrotron DESY, Hamburg and Zeuthen, Germany

${ }^{47}$ Lehrstuhl für Experimentelle Physik IV, Technische Universität Dortmund, Dortmund, Germany

${ }^{48}$ Institut für Kern- und Teilchenphysik, Technische Universität Dresden, Dresden, Germany

${ }^{49}$ Department of Physics, Duke University, Durham, NC, USA

${ }^{50}$ SUPA - School of Physics and Astronomy, University of Edinburgh, Edinburgh, UK

${ }^{51}$ INFN e Laboratori Nazionali di Frascati, Frascati, Italy

52 Physikalisches Institut, Albert-Ludwigs-Universität Freiburg, Freiburg, Germany

${ }^{53}$ II. Physikalisches Institut, Georg-August-Universität Göttingen, Göttingen, Germany

${ }^{54}$ Département de Physique Nucléaire et Corpusculaire, Université de Genève, Genève, Switzerland

55 (a) Dipartimento di Fisica, Università di Genova, Genova, ; ${ }^{(b)}$ INFN Sezione di Genova, Genoa, Italy

${ }^{56}$ II. Physikalisches Institut, Justus-Liebig-Universität Giessen, Giessen, Germany

${ }^{57}$ SUPA - School of Physics and Astronomy, University of Glasgow, Glasgow, UK

${ }^{58}$ LPSC, Université Grenoble Alpes, CNRS/IN2P3, Grenoble INP, Grenoble, France

${ }^{59}$ Laboratory for Particle Physics and Cosmology, Harvard University, Cambridge, MA, USA

60 (a) Department of Modern Physics and State Key Laboratory of Particle Detection and Electronics, University of Science and Technology of China, Hefei, China; ${ }^{(b)}$ Institute of Frontier and Interdisciplinary Science and Key Laboratory of Particle Physics and Particle Irradiation (MOE), Shandong University, Qingdao, China; ${ }^{(c)}$ School of Physics and Astronomy, Shanghai Jiao Tong University, KLPPAC-MoE, SKLPPC, Shanghai, China; ${ }^{(d)}$ Tsung-Dao Lee Institute, Shanghai, China

61 (a) Kirchhoff-Institut für Physik, Ruprecht-Karls-Universität Heidelberg, Heidelberg, Germany; ${ }^{(b)}$ Physikalisches Institut, Ruprecht-Karls-Universität Heidelberg, Heidelberg, Germany

${ }^{62}$ Faculty of Applied Information Science, Hiroshima Institute of Technology, Hiroshima, Japan

63 (a) Department of Physics, Chinese University of Hong Kong, Shatin, N.T., Hong Kong, China; ${ }^{(b)}$ Department of Physics, University of Hong Kong, Hong Kong, China; ${ }^{(c)}$ Department of Physics and Institute for Advanced Study, Hong Kong University of Science and Technology, Clear Water Bay, Kowloon, Hong Kong, China

${ }^{64}$ Department of Physics, National Tsing Hua University, Hsinchu, Taiwan

${ }^{65}$ IJCLab, Université Paris-Saclay, CNRS/IN2P3, 91405, Orsay, France

${ }^{66}$ Department of Physics, Indiana University, Bloomington, IN, USA 
67 (a) INFN Gruppo Collegato di Udine, Sezione di Trieste, Udine, Italy; ${ }^{(b)}$ ICTP, Trieste, Italy; ${ }^{(c)}$ Dipartimento Politecnico di Ingegneria e Architettura, Università di Udine, Udine, Italy

68 (a) INFN Sezione di Lecce, Zona Monte, Italy; ${ }^{(b)}$ Dipartimento di Matematica e Fisica, Università del Salento, Lecce, Italy

69 (a) INFN Sezione di Milano, Milano, Italy; ${ }^{(b)}$ Dipartimento di Fisica, Università di Milano, Milano, Italy

70 (a) INFN Sezione di Napoli, Naples, Italy; ${ }^{(b)}$ Dipartimento di Fisica, Università di Napoli, Napoli, Italy

71 (a) INFN Sezione di Pavia, Pavia, Italy; ${ }^{\text {(b) }}$ Dipartimento di Fisica, Università di Pavia, Pavia, Italy

72 (a) INFN Sezione di Pisa, Pisa, Italy; ${ }^{\text {(b) }}$ Dipartimento di Fisica E. Fermi, Università di Pisa, Pisa, Italy

73 (a) INFN Sezione di Roma, Rome, Italy; ${ }^{\text {(b) }}$ Dipartimento di Fisica, Sapienza Università di Roma, Rome, Italy

74 (a) INFN Sezione di Roma Tor Vergata, Rome, Italy; ${ }^{(b)}$ Dipartimento di Fisica, Università di Roma Tor Vergata, Rome, Italy

75 (a) INFN Sezione di Roma Tre, Rome, Italy; ${ }^{(b)}$ Dipartimento di Matematica e Fisica, Università Roma Tre, Rome, Italy

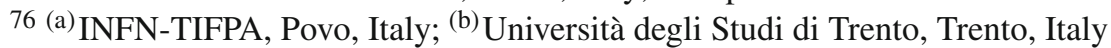

${ }^{77}$ Institut für Astro- und Teilchenphysik, Leopold-Franzens-Universität, Innsbruck, Austria

${ }^{78}$ University of Iowa, Iowa City, IA, USA

${ }^{79}$ Department of Physics and Astronomy, Iowa State University, Ames, IA, USA

${ }^{80}$ Joint Institute for Nuclear Research, Dubna, Russia

81 (a) Departamento de Engenharia Elétrica, Universidade Federal de Juiz de Fora (UFJF), Juiz de Fora, Brazil

; ${ }^{(b)}$ Universidade Federal do Rio De Janeiro COPPE/EE/IF, Rio de Janeiro, Brazil; ${ }^{(c)}$ Universidade Federal de São João del Rei (UFSJ), São João del Rei, Brazil; (d) Instituto de Física, Universidade de São Paulo, São Paulo, Brazil

82 KEK, High Energy Accelerator Research Organization, Tsukuba, Japan

${ }^{83}$ Graduate School of Science, Kobe University, Kobe, Japan

84 (a) AGH University of Science and Technology, Faculty of Physics and Applied Computer Science, Krakow, Poland

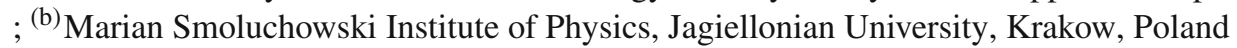

${ }^{85}$ Institute of Nuclear Physics Polish Academy of Sciences, Krakow, Poland

${ }^{86}$ Faculty of Science, Kyoto University, Kyoto, Japan

${ }^{87}$ Kyoto University of Education, Kyoto, Japan

${ }^{88}$ Research Center for Advanced Particle Physics and Department of Physics, Kyushu University, Fukuoka, Japan

${ }^{89}$ Instituto de Física La Plata, Universidad Nacional de La Plata and CONICET, La Plata, Argentina

${ }^{90}$ Physics Department, Lancaster University, Lancaster, UK

${ }^{91}$ Oliver Lodge Laboratory, University of Liverpool, Liverpool, UK

92 Department of Experimental Particle Physics, Jožef Stefan Institute and Department of Physics, University of Ljubljana, Ljubljana, Slovenia

${ }^{93}$ School of Physics and Astronomy, Queen Mary University of London, London, UK

${ }^{94}$ Department of Physics, Royal Holloway University of London, Egham, UK

${ }^{95}$ Department of Physics and Astronomy, University College London, London, UK

${ }^{96}$ Louisiana Tech University, Ruston, LA, USA

${ }^{97}$ Fysiska institutionen, Lunds universitet, Lund, Sweden

${ }^{98}$ Centre de Calcul de l'Institut National de Physique Nucléaire et de Physique des Particules (IN2P3), Villeurbanne, France

${ }^{99}$ Departamento de Física Teorica C-15 and CIAFF, Universidad Autónoma de Madrid, Madrid, Spain

${ }^{100}$ Institut für Physik, Universität Mainz, Mainz, Germany

${ }^{101}$ School of Physics and Astronomy, University of Manchester, Manchester, UK

102 CPPM, Aix-Marseille Université, CNRS/IN2P3, Marseille, France

${ }^{103}$ Department of Physics, University of Massachusetts, Amherst, MA, USA

${ }^{104}$ Department of Physics, McGill University, Montreal, QC, Canada

${ }^{105}$ School of Physics, University of Melbourne, Victoria, Australia

${ }^{106}$ Department of Physics, University of Michigan, Ann Arbor, MI, USA

${ }^{107}$ Department of Physics and Astronomy, Michigan State University, East Lansing, MI, USA

108 B.I. Stepanov Institute of Physics, National Academy of Sciences of Belarus, Minsk, Belarus

${ }^{109}$ Research Institute for Nuclear Problems of Byelorussian State University, Minsk, Belarus

${ }^{110}$ Group of Particle Physics, University of Montreal, Montreal, QC, Canada

${ }^{111}$ P.N. Lebedev Physical Institute of the Russian Academy of Sciences, Moscow, Russia 
112 National Research Nuclear University MEPhI, Moscow, Russia

${ }^{113}$ D.V. Skobeltsyn Institute of Nuclear Physics, M.V. Lomonosov Moscow State University, Moscow, Russia

${ }^{114}$ Fakultät für Physik, Ludwig-Maximilians-Universität München, München, Germany

${ }^{115}$ Max-Planck-Institut für Physik (Werner-Heisenberg-Institut), München, Germany

${ }^{116}$ Nagasaki Institute of Applied Science, Nagasaki, Japan

117 Graduate School of Science and Kobayashi-Maskawa Institute, Nagoya University, Nagoya, Japan

${ }^{118}$ Department of Physics and Astronomy, University of New Mexico, Albuquerque, NM, USA

${ }^{119}$ Institute for Mathematics, Astrophysics and Particle Physics, Radboud University Nijmegen/Nikhef, Nijmegen, The Netherlands

${ }^{120}$ Nikhef National Institute for Subatomic Physics and University of Amsterdam, Amsterdam, The Netherlands

${ }^{121}$ Department of Physics, Northern Illinois University, DeKalb, IL, USA

122 (a) Budker Institute of Nuclear Physics and NSU, SB RAS, Novosibirsk, Russia; ${ }^{(b)}$ Novosibirsk State University

Novosibirsk, Novosibirsk, Russia

${ }^{123}$ Institute for High Energy Physics of the National Research Centre Kurchatov Institute, Protvino, Russia

${ }^{124}$ Institute for Theoretical and Experimental Physics named by A.I. Alikhanov of National Research Centre "Kurchatov

Institute", Moscow, Russia

125 Department of Physics, New York University, New York, NY, USA

${ }^{126}$ Ochanomizu University, Otsuka, Bunkyo-ku, Tokyo, Japan

127 Ohio State University, Columbus, OH, USA

${ }^{128}$ Faculty of Science, Okayama University, Okayama, Japan

${ }^{129}$ Homer L. Dodge Department of Physics and Astronomy, University of Oklahoma, Norman, OK, USA

${ }^{130}$ Department of Physics, Oklahoma State University, Stillwater, OK, USA

${ }^{131}$ Palacký University, RCPTM, Joint Laboratory of Optics, Olomouc, Czech Republic

132 Institute for Fundamental Science, University of Oregon, Eugene, OR, USA

${ }^{133}$ Graduate School of Science, Osaka University, Osaka, Japan

${ }^{134}$ Department of Physics, University of Oslo, Oslo, Norway

135 Department of Physics, Oxford University, Oxford, UK

${ }^{136}$ LPNHE, Sorbonne Université, Université de Paris, CNRS/IN2P3, Paris, France

${ }^{137}$ Department of Physics, University of Pennsylvania, Philadelphia, PA, USA

${ }^{138}$ Konstantinov Nuclear Physics Institute of National Research Centre "Kurchatov Institute", PNPI, St. Petersburg, Russia

${ }^{139}$ Department of Physics and Astronomy, University of Pittsburgh, Pittsburgh, PA, USA

140 (a) Laboratório de Instrumentação e Física Experimental de Partículas - LIP, Lisboa, Portugal; ${ }^{\left({ }^{b}\right)}$ Departamento de Física, Faculdade de Ciências, Universidade de Lisboa, Lisboa, Portugal; ${ }^{(c)}$ Departamento de Física, Universidade de Coimbra, Coimbra, Portugal; ${ }^{(d)}$ Centro de Física Nuclear da Universidade de Lisboa, Lisboa, Portugal; ${ }^{(e)}$ Departamento de Física, Universidade do Minho, Braga, Portugal; ${ }^{(\mathrm{f})}$ Departamento de FA-sica TeA3rica y del Cosmos, Universidad de Granada, Granada, Spain; ${ }^{(\mathrm{g})}$ Dep Física and CEFITEC of Faculdade de Ciências e Tecnologia, Universidade Nova de Lisboa, Caparica, Portugal; ${ }^{\text {(h) }}$ Instituto Superior Técnico, Universidade de Lisboa, Lisboa, Portugal

${ }^{141}$ Institute of Physics of the Czech Academy of Sciences, Prague, Czech Republic

${ }^{142}$ Czech Technical University in Prague, Prague, Czech Republic

${ }^{143}$ Charles University, Faculty of Mathematics and Physics, Prague, Czech Republic

${ }^{144}$ Particle Physics Department, Rutherford Appleton Laboratory, Didcot, UK

145 IRFU, CEA, Université Paris-Saclay, Gif-sur-Yvette, France

${ }^{146}$ Santa Cruz Institute for Particle Physics, University of California Santa Cruz, Santa Cruz, CA, USA

147 (a) Departamento de Física, Pontificia Universidad Católica de Chile, Santiago, Chile; ${ }^{(b)}$ Universidad Andres Bello, Department of Physics, Santiago, ; ${ }^{(c)}$ Instituto de Alta Investigación, Universidad de Tarapacá, Arica, Chile

; (d) Departamento de Física, Universidad Técnica Federico Santa María, Valparaíso, Chile

${ }^{148}$ Department of Physics, University of Washington, Seattle, WA, USA

${ }^{149}$ Department of Physics and Astronomy, University of Sheffield, Sheffield, UK

${ }^{150}$ Department of Physics, Shinshu University, Nagano, Japan

${ }^{151}$ Department Physik, Universität Siegen, Siegen, Germany

152 Department of Physics, Simon Fraser University, Burnaby, BC, Canada

${ }^{153}$ SLAC National Accelerator Laboratory, Stanford, CA, USA

${ }^{154}$ Physics Department, Royal Institute of Technology, Stockholm, Sweden 
155 Departments of Physics and Astronomy, Stony Brook University, Stony Brook, NY, USA

${ }^{156}$ Department of Physics and Astronomy, University of Sussex, Brighton, UK

157 School of Physics, University of Sydney, Sydney, Australia

158 Institute of Physics, Academia Sinica, Taipei, Taiwan

$159{ }^{(a)}$ E. Andronikashvili Institute of Physics, Iv. Javakhishvili Tbilisi State University, Tbilisi, Georgia; ${ }^{(b)}$ High Energy

Physics Institute, Tbilisi State University, Tbilisi, Georgia

${ }^{160}$ Department of Physics, Technion, Israel Institute of Technology, Haifa, Israel

${ }^{161}$ Raymond and Beverly Sackler School of Physics and Astronomy, Tel Aviv University, Tel Aviv, Israel

162 Department of Physics, Aristotle University of Thessaloniki, Thessaloniki, Greece

${ }^{163}$ International Center for Elementary Particle Physics and Department of Physics, University of Tokyo, Tokyo, Japan

${ }^{164}$ Graduate School of Science and Technology, Tokyo Metropolitan University, Tokyo, Japan

165 Department of Physics, Tokyo Institute of Technology, Tokyo, Japan

166 Tomsk State University, Tomsk, Russia

167 Department of Physics, University of Toronto, Toronto, ON, Canada

168 (a) TRIUMF, Vancouver, BC, Canada; ${ }^{(b)}$ Department of Physics and Astronomy, York University, Toronto, ON, Canada

${ }^{169}$ Division of Physics and Tomonaga Center for the History of the Universe, Faculty of Pure and Applied Sciences,

University of Tsukuba, Tsukuba, Japan

${ }^{170}$ Department of Physics and Astronomy, Tufts University, Medford, MA, USA

${ }^{171}$ Department of Physics and Astronomy, University of California Irvine, Irvine, CA, USA

172 Department of Physics and Astronomy, University of Uppsala, Uppsala, Sweden

173 Department of Physics, University of Illinois, Urbana, IL, USA

${ }^{174}$ Instituto de Física Corpuscular (IFIC), Centro Mixto Universidad de Valencia - CSIC, Valencia, Spain

175 Department of Physics, University of British Columbia, Vancouver, BC, Canada

${ }^{176}$ Department of Physics and Astronomy, University of Victoria, Victoria, BC, Canada

${ }^{177}$ Fakultät für Physik und Astronomie, Julius-Maximilians-Universität Würzburg, Würzburg, Germany

${ }^{178}$ Department of Physics, University of Warwick, Coventry, UK

${ }^{179}$ Waseda University, Tokyo, Japan

${ }^{180}$ Department of Particle Physics, Weizmann Institute of Science, Rehovot, Israel

${ }^{181}$ Department of Physics, University of Wisconsin, Madison, WI, USA

${ }^{182}$ Fakultät für Mathematik und Naturwissenschaften, Fachgruppe Physik, Bergische Universität Wuppertal, Wuppertal, Germany

${ }^{183}$ Department of Physics, Yale University, New Haven, CT, USA

184 Yerevan Physics Institute, Yerevan, Armenia

${ }^{a}$ Also at Borough of Manhattan Community College, City University of New York, New York NY, USA

${ }^{b}$ Also at CERN, Geneva, Switzerland

${ }^{c}$ Also at CPPM, Aix-Marseille Université, CNRS/IN2P3, Marseille, France

${ }^{\mathrm{d}}$ Also at Département de Physique Nucléaire et Corpusculaire, Université de Genève, Genève, Switzerland

e Also at Departament de Fisica de la Universitat Autonoma de Barcelona, Barcelona, Spain

${ }^{\mathrm{f}}$ Also at Department of Applied Physics and Astronomy, University of Sharjah, Sharjah, United Arab Emirates,

g Also at Department of Financial and Management Engineering, University of the Aegean, Chios, Greece

${ }^{\mathrm{h}}$ Also at Department of Physics and Astronomy, Michigan State University, East Lansing MI, USA

${ }^{\mathrm{i}}$ Also at Department of Physics and Astronomy, University of Louisville, Louisville, KY, USA

${ }^{j}$ Also at Department of Physics, Ben Gurion University of the Negev, Beer Sheva, Israel

${ }^{\mathrm{k}}$ Also at Department of Physics, California State University, East Bay, USA

${ }^{1}$ Also at Department of Physics, California State University, Fresno, USA

${ }^{m}$ Also at Department of Physics, California State University, Sacramento, USA

${ }^{n}$ Also at Department of Physics, King's College London, London, UK

${ }^{\circ}$ Also at Department of Physics, St. Petersburg State Polytechnical University, St. Petersburg, Russia

p Also at Department of Physics, Stanford University, Stanford CA, USA

${ }^{\mathrm{q}}$ Also at Department of Physics, University of Adelaide, Adelaide, Australia

${ }^{\mathrm{r}}$ Also at Department of Physics, University of Fribourg, Fribourg, Switzerland

${ }^{s}$ Also at Department of Physics, University of Michigan, Ann Arbor MI, USA 
${ }^{\mathrm{t}}$ Also at Dipartimento di Matematica, Informatica e Fisica, Università di Udine, Udine, Italy

u Also at Faculty of Physics, M.V. Lomonosov Moscow State University, Moscow, Russia

${ }^{v}$ Also at Giresun University, Faculty of Engineering, Giresun, Turkey

${ }^{w}$ Also at Graduate School of Science, Osaka University, Osaka, Japan

${ }^{x}$ Also at Hellenic Open University, Patras, Greece

y Also at IJCLab, Université Paris-Saclay, CNRS/IN2P3, 91405, Orsay, France

${ }^{\mathrm{z}}$ Also at Institucio Catalana de Recerca i Estudis Avancats, ICREA, Barcelona, Spain

aa Also at Institut für Experimentalphysik, Universität Hamburg, Hamburg, Germany

${ }^{\text {ab }}$ Also at Institute for Mathematics, Astrophysics and Particle Physics, Radboud University Nijmegen/Nikhef, Nijmegen, Netherlands

${ }^{\text {ac }}$ Also at Institute for Nuclear Research and Nuclear Energy (INRNE) of the Bulgarian Academy of Sciences, Sofia, Bulgaria

${ }^{\text {ad }}$ Also at Institute for Particle and Nuclear Physics, Wigner Research Centre for Physics, Budapest, Hungary

ae Also at Institute of Particle Physics (IPP), Vancouver, Canada

af Also at Institute of Physics, Academia Sinica, Taipei, Taiwan

ag Also at Institute of Physics, Azerbaijan Academy of Sciences, Baku, Azerbaijan

ah Also at Institute of Theoretical Physics, Ilia State University, Tbilisi, Georgia

ai Also at Instituto de Fisica Teorica, IFT-UAM/CSIC, Madrid, Spain

aj Also at Joint Institute for Nuclear Research, Dubna, Russia

ak Also at Louisiana Tech University, Ruston LA, USA

al Also at LPNHE, Sorbonne Université, Université de Paris, CNRS/IN2P3, Paris, France

am Also at Manhattan College, New York NY, USA

an Also at Moscow Institute of Physics and Technology State University, Dolgoprudny, Russia

ao Also at National Research Nuclear University MEPhI, Moscow, Russia

ap Also at Physics Department, An-Najah National University, Nablus, Palestine

aq Also at Physics Dept, University of South Africa, Pretoria, South Africa

${ }^{a r}$ Also at Physikalisches Institut, Albert-Ludwigs-Universität Freiburg, Freiburg, Germany

${ }^{a}$ Also at School of Physics, Sun Yat-sen University, Guangzhou, China

at Also at The City College of New York, New York NY, USA

${ }^{\text {au }}$ Also at TRIUMF, Vancouver BC, Canada

${ }^{\text {av }}$ Also at Universita di Napoli Parthenope, Napoli, Italy.

Deceased: O. Igonkina, B.T. King, D. Lellouch. 